\title{
Target Tracking Using Various Filters in Synthetic Aperture Radar Data and Imagery
}

\author{
A Thesis \\ Presented to the Faculty of \\ California Polytechnic State University \\ San Luis Obispo, California
}

\author{
In Partial Fulfillment \\ Of the Requirements for the Degree \\ Master of Science in Electrical Engineering
}

By

Jessica Kiefer

May 2009 
(C) 2009

Jessica Kiefer

ALL RIGHTS RESERVED 


\section{Committee Membership}

TITLE: Target Tracking Using Various Filters in Synthetic Aperture Data and Imagery AUTHOR: Jessica Kiefer

DATE SUBMITTED: May 2009

COMMITTEE CHAIR: Dr. John Saghri, Associate Professor

COMMITTEE MEMBER: Dr. Helen Yu, Associate Professor

COMMITTEE MEMBER: Dr. Wayne Pilkington, Assistant Professor 


\section{Abstract}

\section{Target Tracking Using Various Filters in Synthetic Aperture Radar Imagery}

\section{Jessica Kiefer}

This thesis explores the use and accuracy of several discrete-time image filters for the purpose of target tracking in Synthetic Aperture Radar imagery. Both extended targets and point targets are used for tracking, showing the need for different types of filters for each target type.

Monte Carlo analysis is performed on the results of the extended target filter results to determine the absolute mean-squared error between the filter prediction of the target centroid and the actual location of the target centroid. Two different filters were chosen for the extended target: Kalman and $\mathrm{H}$ Infinity.

Both the Kalman and $\mathrm{H}$ Infinity filters perform tracking by accurately estimating the state of the dynamic system, and in some cases it may be useful to simulate a situation when a target temporarily disappears from radar view. The ability of both filters to predict target location with no input measurements is investigated. A unique trait of the $\mathrm{H}$ Infinity filter is its ability to accurately and efficiently estimate the state of a dynamic system given no information about the noise environment.

To simulate more realistic targets, smaller circular and square targets are created and a sensitivity analysis is performed using the Kalman and $\mathrm{H}$ Infinity filters to determine the shortfalls of these filter techniques as targets become smaller and smaller. The results 
indicate that these tracking methods are no longer useful as the targets become so small that they approach being only a single pixel in size.

A new filter called the Prediction and Matching Detection (PAMD) filter is used for single-pixel point targets. This filter illustrates the importance of having very high frame rate images with little change in velocity over consecutive frames if choosing to use the PAMD algorithm. The PAMD filter is extended to track more than one target at a time.

Tracking of raw SAR data is preferred over post-processed images due to the decreased amount of processing time. The Kalman and $\mathrm{H}$ Infinity filters are implemented to track raw radar data during its first 3 seconds of motion in 2-dimensions by accounting for the measurements of two parameters: the squint angle and slant range. Noise is added to the measurements to simulate platform inaccuracies.

The project is a continuation of prior SAR research at Cal Poly under Dr. John Saghri with the sponsorship of Raytheon Space \& Airborne Systems. 


\section{Acknowledgements}

I would like to thank my advisor, Dr John Saghri, and Raytheon Space and Airborne Systems for their continued support and excitement towards all of the students completing research with them. Working with them has been a wonderful experience. In addition, I would like to thank my thesis committee members Dr Wayne Pilkington and Dr Helen Yu for their valuable feedback.

I would also like to thank my family for supporting me throughout my time at Cal Poly-I would never have been successful without their love and encouragement!

I would like to thank all of the wonderful faculty and support staff in the Electrical Engineering department for their time and dedication throughout the past 5 years.

Finally, I would like to thank my friends, lab partners, project group members, and all of my other peers who have made my college experience fun and exciting. 


\section{Table of Contents}

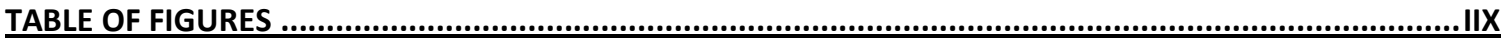

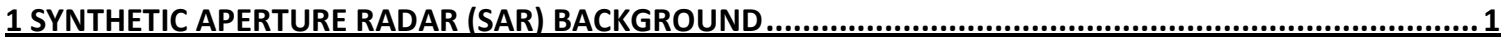

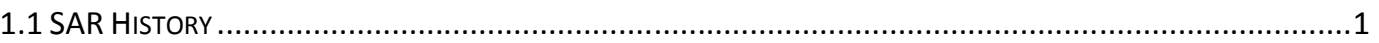

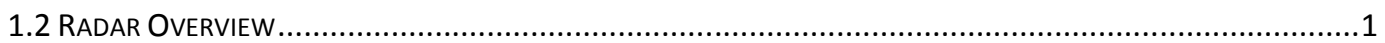

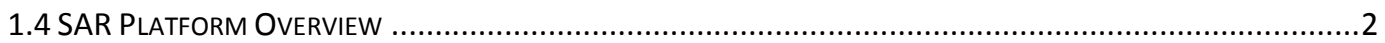

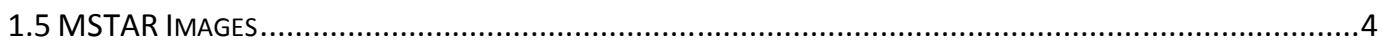

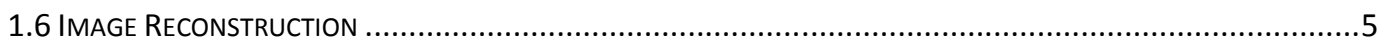

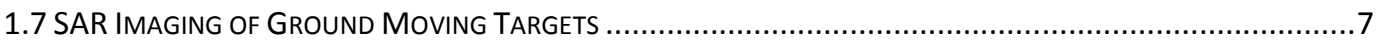

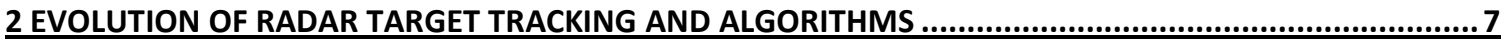

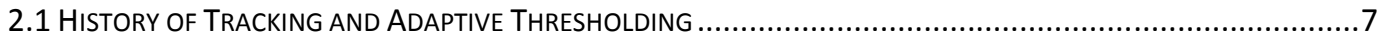

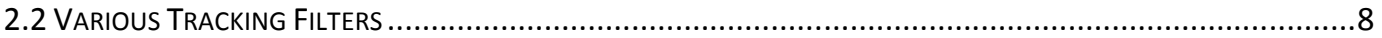

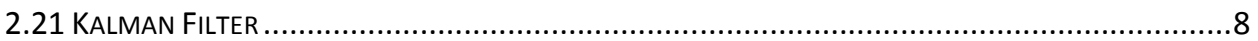

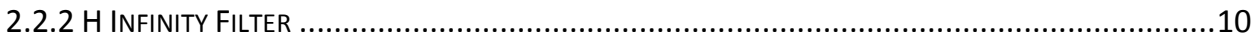

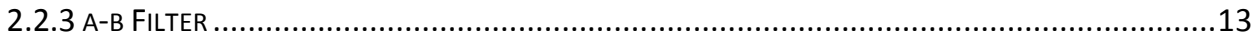

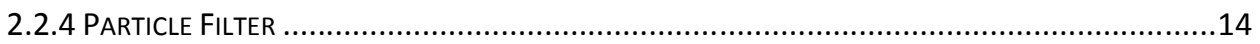

2.2.5 VARIATIONS OF THE KALMAN FILTER .................................................................. 16

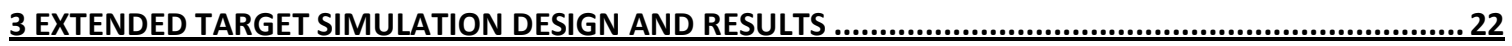

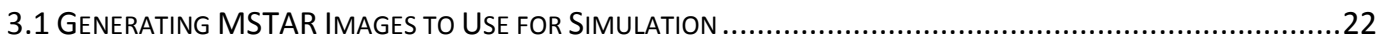

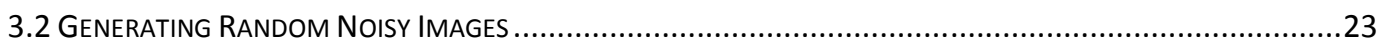

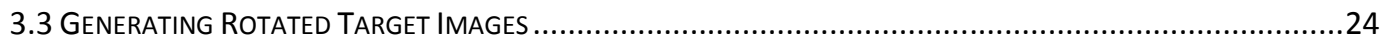

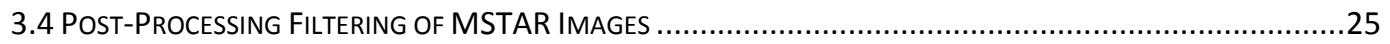

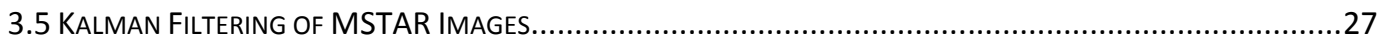

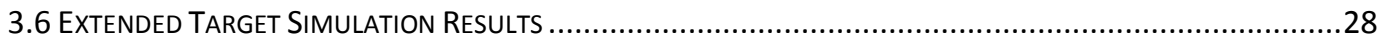

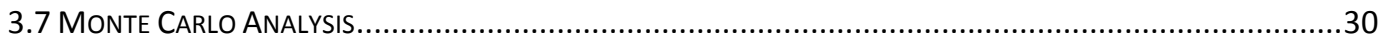

3.7.1 MonTE CARLO ANALYSIS OF KALMAN FILTER ..................................................... 31

3.7.2 Monte CARLo ANAlysis of H INFINITY FILTER .......................................................33

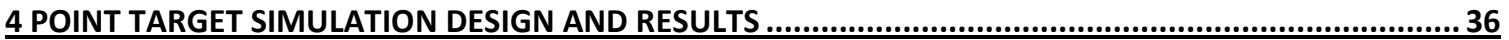

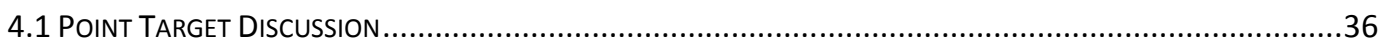

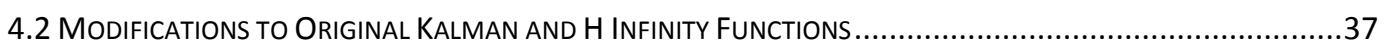

4.3 KALMAN AND H-INFINITY FILTER RESULTS FOR CIRCULAR AND SQUARE TARGETS.....................................38 


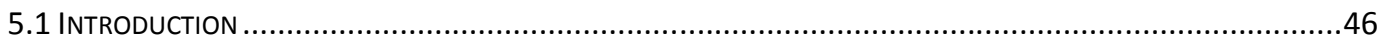

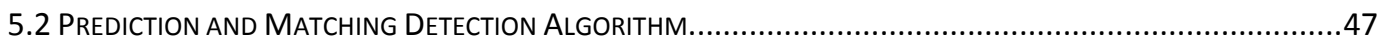

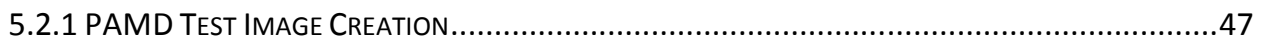

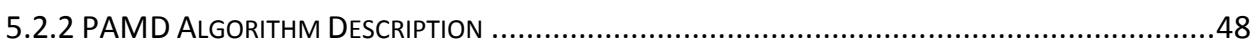

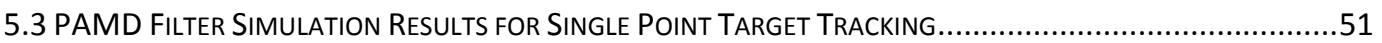

5.4 PAMD Filter Simulation Results for Multiple Point TARget Tracking......................................53

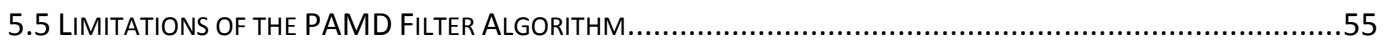

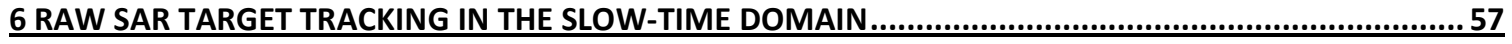

6.1 MOVING TARGET IMAGING WITHOUT COMPENSATION ..............................................................5

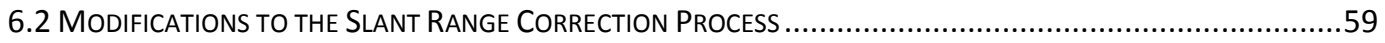

6.3 SAR TARGET TRACKING USING SQUINT ANGLE AND SLANT RANGE ..................................................64

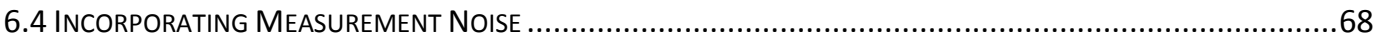

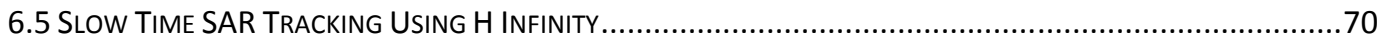

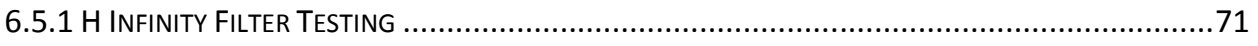

6.5.2 SLOW-TIME FILTER TYPE COMPARISON .......................................................... 72

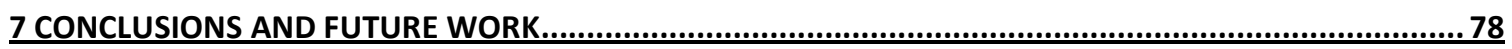

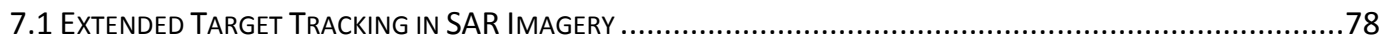

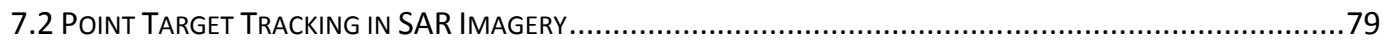

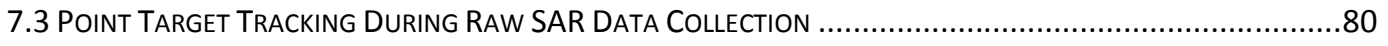

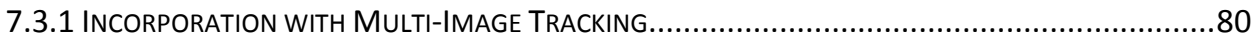

7.3.2 NON-CONSTANT MOVEMENT DURING RAW DATA COLLECTION ........................................ 81

7.3.3 INCORPORATION WITH AUTOMATIC TARGET RECOGNITION ...........................................81

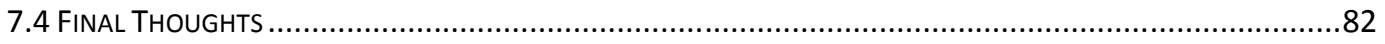

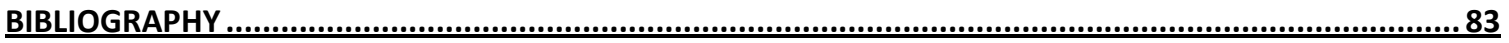

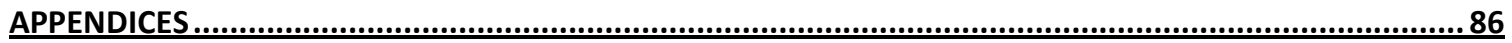

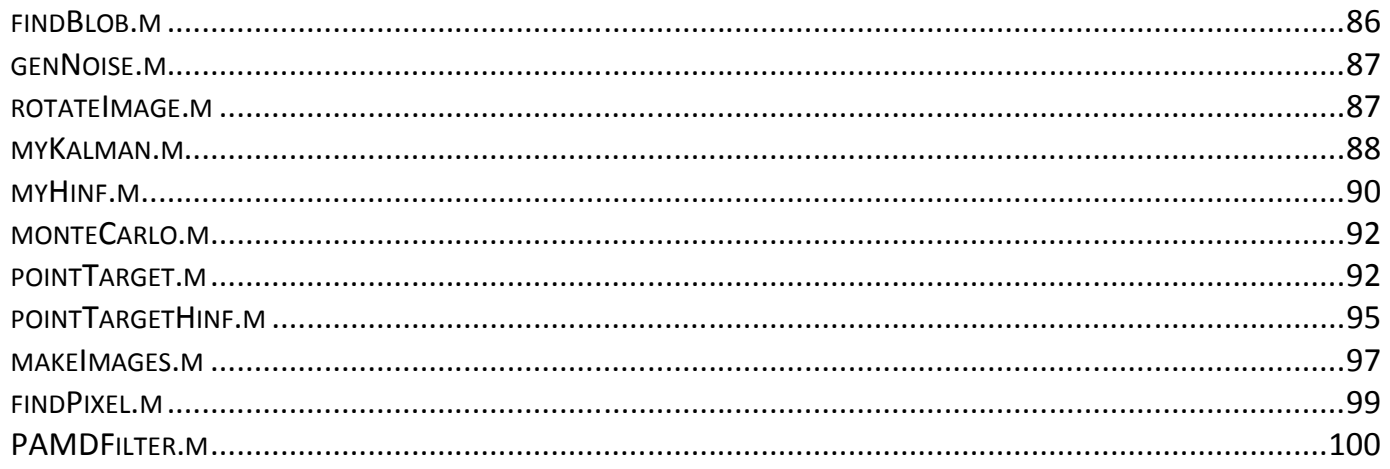




\section{Table of Figures}

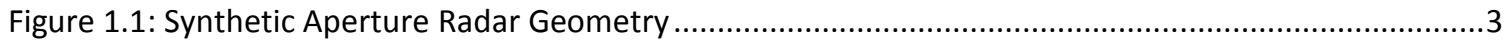

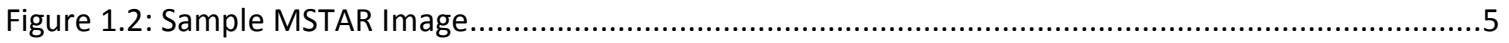

Figure 1.3: SAR Image Reconstruction Signals and Variables...........................................................6

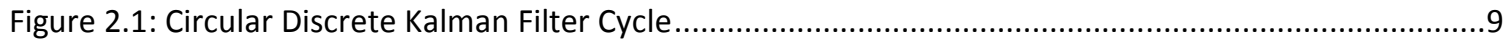

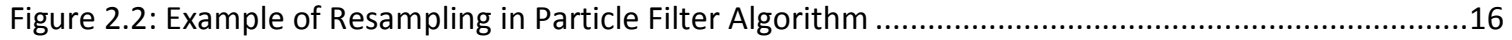

Figure 2.3: Variations of the Kalman Filter ................................................................................

Figure 2.4: Measurement update equation of the standard Kalman filter showing $r x \mathrm{r}$ matrix inversion ...18

Figure 2.5: Measurement update equation of sequential Kalman filter showing $r$ scalar divisions .............18

Figure 3.1: Sample MSTAR image including random background and rotated target ..............................22

Figure 3.2: Sample Noisy Background Image generated using genNo ise . m........................................24

Figure 3.3: Sample targets rotated between -10 degrees and +10 degrees ..........................................25

Figure 3.4 - Threshold Step of Post-Processing ................................................................................26

Figure 3.5 - Fully-Filtered Image of Target showing an ' $x$ ' where the centroid is located ...........................26

Figure 3.6 - Sample Kalman Algorithm Image Showing True and Kalman Estimated State .........................27

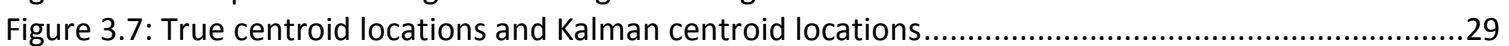

Figure 3.8: True centroid locations and $\mathrm{H}$ Infinity centroid locations ......................................................29

Figure 3.9: $P$ and $Q$ Values for Monte Carlo Analysis using the Kalman filter............................................31

Figure 3.10: Mean-Squared Errors for Row Pixels..............................................................................3

Figure 3.11: Mean-Squared Errors for Column Pixels .........................................................................32

Figure 3.12: Monte Carlo Analysis Parameters for H Infinity Filter...................................................33

Figure 3.13: Mean-Squared Error for Row Pixels with H Infinity Filter ...............................................33

Figure 3.14: Mean-Squared Error for Column Pixels with H Infinity Filter .............................................34

Figure 3.15: Sample of large mean-squared error graphical results for $\mathrm{H}$ Infinity filter ............................35

Figure 4.1: Sample Test Image for Circular Target of Radius 4 Pixels....................................................3

Figure 4.2: Sample Test Image for Square Target of Length 4 Pixels ....................................................3

Figure 4.3: Sample Image Showing Incorrectly Located Circular Target Due to Background Noise .............39

Figure 4.4: Awry Kalman Filter Example for Circular Target.................................................................40

Figure 4.5: Awry H Infinity Filter Example for Circular Target ..........................................................41

Figure 4.6: Simulated Point Targets for Kalman Filter with 5\% Noise ....................................................42

Figure 4.7: Simulated Point Targets for Kalman Filter with 7.5\% Noise ...................................................42

Figure 4.8: Simulated Point Targets for Kalman Filter with 10\% Noise .................................................43

Figure 4.9: Simulated Point Targets for $\mathrm{H}$ Infinity Filter with $5 \%$ Noise ................................................43

Figure 4.10: Simulated Point Targets for $\mathrm{H}$ Infinity Filter with 7.5\% Noise ..............................................44

Figure 4.11: Simulated Point Targets for H Infinity Filter with 10\% Noise ...............................................44

Figure 5.1: Sample Test Image for the PAMD Filter ........................................................................48

Figure 5.2: Predicting Process (Circles represent known position, crosses represent predicted position) ...49

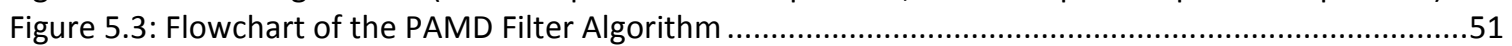

Figure 5.4: PAMD Filter Results ....................................................................................................5

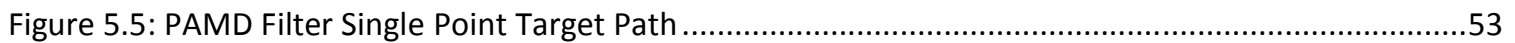

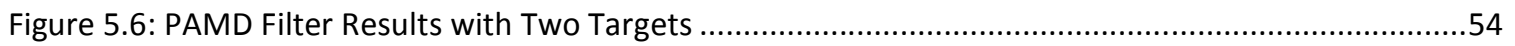

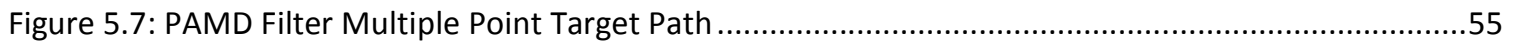

Figure 6.1: Final target image for two moving targets without compensation ........................................58

Figure 6.2: Range tracked position estimates with 2-D motion and only a single input parameter .............61

Figure 6.3: Azimuth tracked position estimates with 2-D motion and only a single input parameter .........61

Figure 6.4: Variable and parameter descriptions for measurement formulas..........................................63

Figure 6.5: Kalman Range Position Estimates for 2-D Moving Target ................................................65

Figure 6.6: Kalman Azimuth Position Estimates for Moving 2-D Target ............................................66

Figure 6.7: Kalman Range Velocity Estimates for Moving 2-D Target ....................................................66

Figure 6.8: Kalman Azimuth Velocity Estimates for Moving 2-D Target.............................................67 


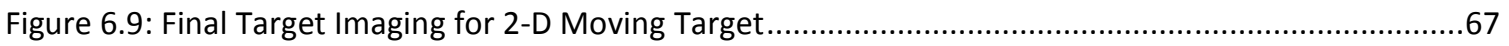

Figure 6.10: Final Target Imaging for 2-D Moving Target -- Zoomed In ................................................68

Figure 6.11: Kalman Range and Position Estimations for 2-D Moving Target .........................................69

Figure 6.12: Kalman Range and Position Velocity Estimations for 2-D Moving Target ................................69

Figure 6.13: Final Target Imaging for 2-D Moving Target with Added Measurement Noise .......................70

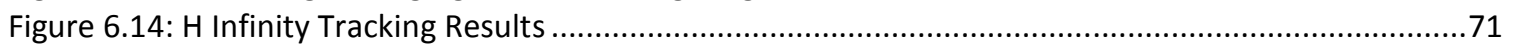

Figure 6.15: Slow-time H Infinity Filter Periodic Track Jumping ...................................................72

Figure 6.16: Mean-Squared Error Performance of Kalman and $\mathrm{H}$ Infinity Filters .....................................74

Figure 6.17: Mean-Squared Error Performance of Kalman and H Infinity Filters ......................................75

Figure 6.18: Mean-Squared Error Performance of Kalman and H Infinity Filters .....................................76

Figure 6.19: Mean-Squared Error Performance for Kalman Slow-Time Filter Performance........................75

Figure 6.20: Mean-Squared Error Performance fore H Infinity Slow-Time Filter Performance ...................76 


\section{Synthetic Aperture Radar (SAR) Background}

\subsection{SAR History}

The realization of Synthetic Aperture Radar (SAR) is often credited to Carl Wiley of Goodyear Aerospace in 1951. SAR is an active microwave remote sensing technology that is used to create two-dimensional images of targets and the Earth's surface through the use of radar ${ }^{[16]}$. The term "synthetic" in the name comes from the concept of effectively creating a very long, narrow antenna by post-processing signal analysis rather than using a physical structure. This yields a much better resolution in the axis perpendicular to the radar beam (called the azimuth axis) effectively yielding images with quality normally only possible by using a much larger physical radar antenna ${ }^{[13]}$.

SAR became an important aspect of remote sensing in the 1970s when SAR technology was introduced to satellite platforms ${ }^{[13]}$. The further increase in the use of SAR is based mostly on the following principles: radar requires no illumination and works well in darkness, the electromagnetic waves of radar frequencies pass through clouds with very minimal deterioration, and radar scatters off targets much differently than optical energy, allowing for a different perspective of surfaces than optical sensors can provide.

\subsection{Radar Overview}

A radio detection and ranging (radar) system uses electromagnetic waves to determine various properties of moving and fixed objects, such as planes, ships, vehicles, terrain, 
and weather patterns. The system that comprises radar is a transmitter that sends microwaves/radio waves which are reflected by the target (and any other object in their path) and then detected and received by a receiver. The returned signal is often weak and requires post-processing to obtain the desired results. Generally, the time it takes for the signal to return to the receiver is used to determine how far away objects in the signal path are ${ }^{[15]}$. This information can also be used to find velocity and dimensions.

\subsection{SAR Platform Overview}

Figure 1.1 shows a geometric model used to describe SAR data acquisition. This model is the assumed platform used in this thesis and describes a moving aerial platform with a side-looking radar antenna ${ }^{[3]}$. Figure 1.1 displays the platform at the point of minimum distance from the target, which is called the point of minimum slant range. This type of SAR platform is referred to as stripmap SAR, in which the radar beam is constantly pointed in the same direction relative to the platform. This means the area illuminated by the beam is constantly changing. 


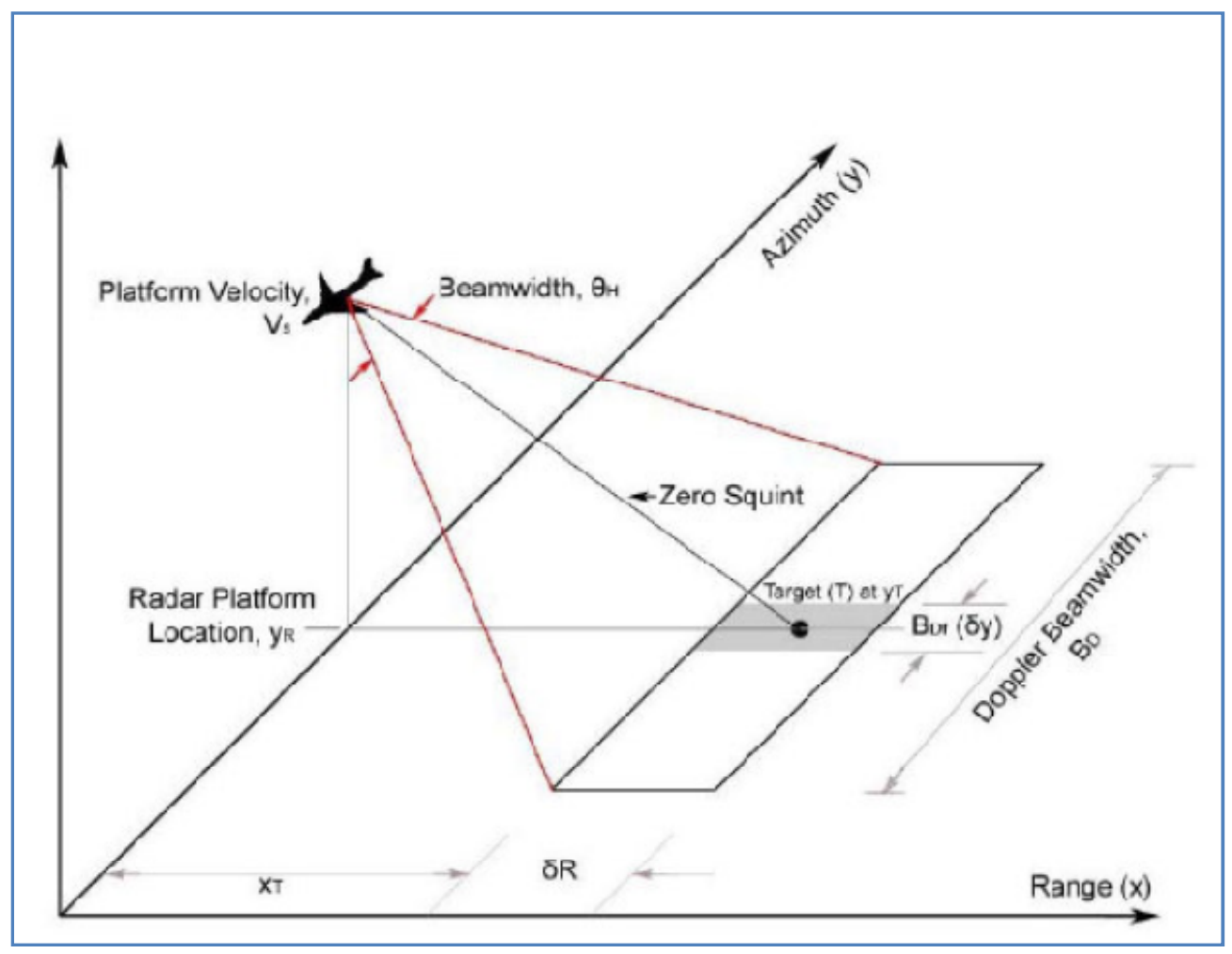

Figure 1.1: Synthetic Aperture Radar Geometry

The following terms are used to describe the geometry in Figure 1:

Azimuth: The azimuth direction refers to the axis in parallel with that of the moving platform, and increases with the direction of the platform.

Beam Footprint: As a pulse is transmitted through space, pulses of electromagnetic energy are emitted towards the ground. This results in the radar antenna projecting a beam onto an area of the ground called beam footprint.

Range: The range direction refers to the direction directly perpendicular to the moving platform, and increases perpendicular away from the platform. 
Squint Angle: The squint angle is the angle measured in the slant range plane that the slant range vector makes with the zero Doppler plane. This angle depends upon the target range for a given beam pointing direction.

\subsection{MSTAR Images}

The Moving and Stationary Target Acquisition and Recognition (MSTAR) data is a large set of public spotlight-mode SAR images of various targets. A portion of this project utilizes this image set for the purpose of tracking both extended targets as well as point targets. The background noise created in this project is actually generated directly from the MSTAR sample images.

The MSTAR images are very low pixel resolution at $128 \times 128$ pixels and are created to have an apparent viewing angle from directly above the target. The approximate physical resolution per pixel is 0.3047 meters with the pixel spacing being 0.2 meters ${ }^{[5]}$.

It is important to note that in an MSTAR image, the extended target is stationary and comprises a fair amount of the image. A sample MSTAR image can be seen below in Figure 1.2. 


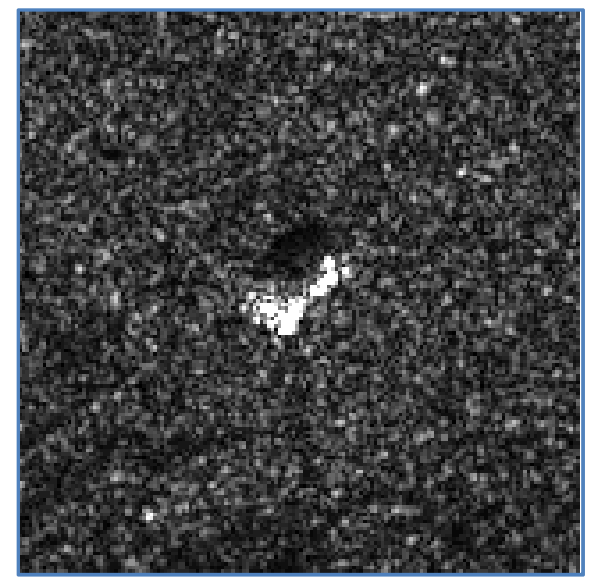

Figure 1.2: Sample MSTAR Image

\subsection{Image Reconstruction}

The creation of a SAR image involves the reconstruction of what's called a target image function. This function represents the radar reflectivity of the target region, and is often called $f(x, y)$ where $x$ and $y$ are the range and azimuth dimensions ${ }^{[5][13]}$.

Mathematically, the reconstruction of a target image function (to create a SAR image), involves using matched filtering with the signal returned to the radar antenna from the target region. This signal, $s(t, u)$, is multiplied in the frequency domain by a modified, phase-shifted version of the complex conjugate of the transmitted radar signal $P(\omega)$. The phase is only shifted when using spotlight-mode SAR where the beam-stearing is used $^{[5][17]}$. Once this matched-filtering is done, the frequency domain version of the reconstructed target function is: ${ }^{[5][13]}$

$$
F\left[k_{x}\left(\omega, k_{u}\right), k_{y}\left(\omega, k_{u}\right)\right]=S\left(\omega, k_{u}\right) \cdot P^{*}(\omega) \exp \left(j k_{x} X_{c}+j k_{y} Y_{c}\right) .
$$


The $k_{x}\left(\omega, k_{u}\right)$ and $k_{y}\left(\omega, k_{u}\right)$ shown in this equation are simple domain transformation functions to place the target function in the $x-y$ Cartesian plane ${ }^{[5][13]}$.

The output SAR image is actually obtained by using the two-dimensional Inverse Fast Fourier Transform (FFT) on the frequency domain function in Equation 1. Figure 1.3 describes all of the signals and variables used in this equation. Fast-time and slow-time refer to the time domains in the range dimension (in the radar beam signal) and azimuth dimension (in the synthetic aperture) ${ }^{[5][13]}$.

\begin{tabular}{|c|c|}
\hline$S\left(\omega, k_{u}\right)$ & The measured radar signal returned to the SAR antenna. \\
\hline$P^{*}(\omega)$ & The matched-filter complex conjugate of the transmitted radar signal. \\
\hline$k_{x}$ & Spatial frequency domain in the range dimension (frequency domain for $t$ ). \\
\hline$k_{y}$ & Spatial frequency domain in the azimuth dimension (frequency domain for $u$ ). \\
\hline$\omega$ & Fast-time frequency domain (frequency domain for $t$ ). \\
\hline$k_{u}$ & Slow-time frequency domain (frequency domain for $u$ ). \\
\hline$X_{c}$ & Range dimension center position of the target region. \\
\hline$Y_{c}$ & Azimuth dimension center position of the target region. \\
\hline
\end{tabular}

Figure 1.3: SAR Image Reconstruction Signals and Variables 


\subsection{SAR Imaging of Ground Moving Targets}

SAR requires data to be taken using multiple receive and transmit antenna positions. For this reason, it is most successfully used over relatively immobile targets.

\section{Evolution of Radar Target Tracking and Algorithms}

\subsection{History of Tracking and Adaptive Thresholding}

Historically, there have been two main goals to military target tracking: the first goal is to predict a target's future location to use for interception/destruction. The second goal is for navigation and collision avoidance, used more often for civilian cases ${ }^{[11]}$.

The oldest method for obtaining tracking information on a target was done manually. An operator would use a grease pencil to manually mark on the monitor the location of the target each time the radar data scan came in. Obviously, this was not very effective and it was soon realized that computers were much more apt for the job of target tracking. This paved the way for Automatic Detection and Tracking (ADT).

The statistical framework necessary for ADT has been around since the 1940s, however the widespread use of ADT was not prevalent until digital processing and computer hardware became more affordable. The first use of ADT was very limited, and system noise was often assumed to be constant, therefore ignoring the fact that a realistic environment was guaranteed to have additional noise that would be nearly impossible to account for ${ }^{[12]}$. 
This paved the way for a new approach to tracking called adaptive thresholding. This new method assumed that the noise density was known, except for a few unknown/changing parameters such as the mean and variance. Other known variables were then used to help estimate unknown values of mean and variance. Spawning from the adaptive thresholding and ADT are the more modern versions of tracking - the numerous named filters used for various applications today.

\subsection{Various Tracking Filters}

Highlighted in this section are more modern filters that are used for tracking of many different targets - not necessarily military targets. Not all of the filters are optimal for use in military grade target tracking. In addition, some filters are adaptive, while others are not.

\subsection{Kalman Filter}

The Kalman filter, discovered in the 1960s by Rudolf Kalman, is a time-domain recursive filter with the ability to estimate the state of a dynamic system by using a series of measurements. The Kalman filter operates by propagating the state mean and covariance through time. The only information required for the filter is the state estimate from the previous time instant and the current measurement; there is no need to keep history of all past state estimates. Kalman filters are most often used for systems which contain large amounts of noise. Some applications of Kalman filters are control theory, radar, and computer vision. To use the Kalman filter to estimate the 
state of a process, one must model the process after the Kalman filtering procedure described in the following section.

From a high level, the Kalman filter uses feedback control to estimate a process ${ }^{[1]}$. The Kalman filter first estimates the state at an instance in time and then obtains feedback in the form of noisy measurements. This can be further broken down into two types of equations: time update equations and measurement update equations. Time update equations propagate the error covariance matrices and current state to obtain a priori measurements for the next set of equations. The measurement update equations are used to incorporate the noisy measurements yielding an improved a posteriori estimate. This discrete process continues until the user chooses to stop it.

The time update equations can be thought of as a form of "predictor" equations, whereas the measurement update equations are a form of "corrector" equations. An illustration of this high-level process can be seen in Figure 2.1.

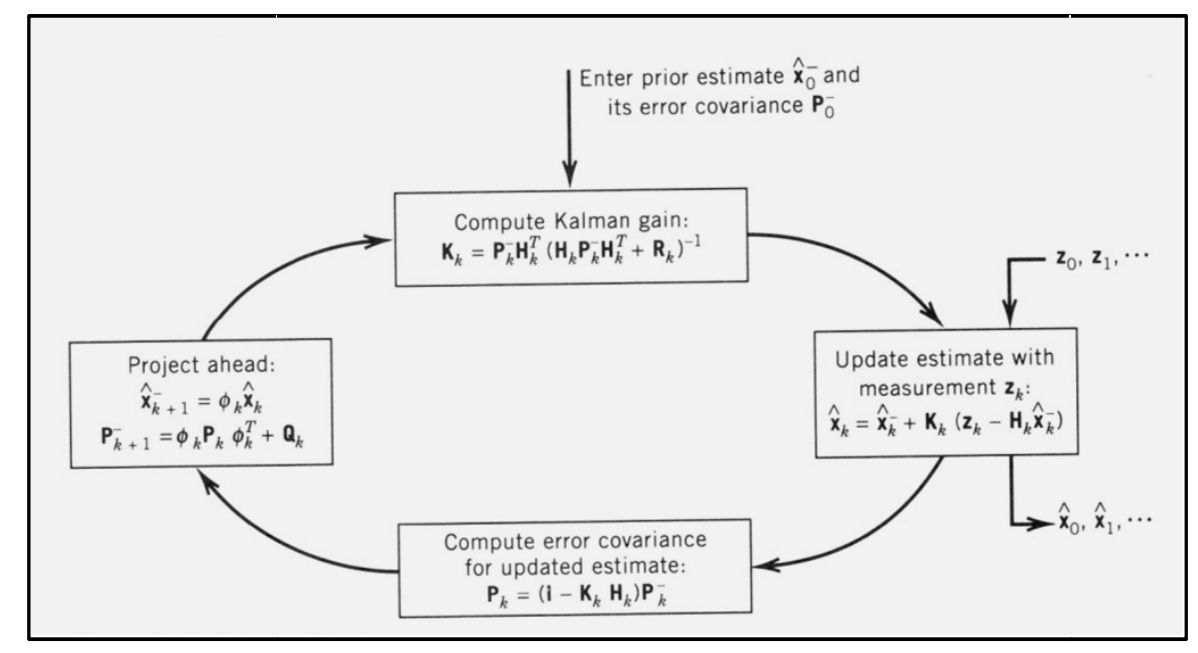

Figure 2.1: Circular Discrete Kalman Filter Cycle 
A summary of the Kalman equations are as follows:

1) Update Kalman Gain Matrix

$$
K_{k}=P_{k}^{-} H_{k}^{T}\left(H_{k} P_{k}^{-} H_{k}^{T}+R_{k}\right)^{-1}
$$

2) Acquire new measurement, $z_{k}$

3) Update State Estimate with new measurement

$$
\hat{x}_{k}=\hat{x}_{k}^{-}+K_{k}\left(z_{k}-H_{k} \hat{x}_{k}^{-}\right)
$$

4) Update Error Covariance matrix

$$
P_{k}=\left(I-K_{k} H_{k}\right) P_{k}^{-}
$$

5) Project ahead and find state based on system dynamics

$$
\hat{x}_{k+1}^{-}=\emptyset_{k} \hat{x}_{k}
$$

6) Project ahead and find the error covariance based on dynamics and expected process noise

$$
P_{k+1}^{-}=\emptyset_{k} P_{k} \emptyset_{k}^{T}+Q_{k}
$$

\subsubsection{H Infinity Filter}

$\mathrm{H}$ infinity filters are a more robust version of the Kalman filter first introduced in 1987 by Mike Grimble, a professor in the UK ${ }^{[9]}$. The Kalman filter relies on many statistical 
quantities attributed to noise being known, and thus defined, in the equations used for processing. However, these statistical quantities are not always known or computable. The $\mathrm{H}$ infinity filter is able to solve the problem of noise uncertainty and its equations do not make any assumptions about the noise present in the system. The $\mathrm{H}$ infinity filter also does not require the noise processes to be zero mean, as does the Kalman filter. One reason the $\mathrm{H}$ infinity filter is not more widely used is its lack of uniformity-there are many different versions of the $\mathrm{H}$ infinity filter available, making it difficult to standardize.

The Kalman filter results in the smallest possible standard deviation of the estimation error. More succinctly, the Kalman filter is the minimum variance estimator. On the other hand, the $\mathrm{H}$ infinity filter does not make assumptions about noise and therefore minimizes the worst case estimation error.

Suppose we have a discrete linear system defined by the following:

$$
\begin{aligned}
& x_{k+1}=A x_{k}+B u_{k}+w_{k} \\
& y_{k}=C x_{k}+z_{k}
\end{aligned}
$$

where $A, B$, and $C$ are known matrices; $k$ is the time index; $x$ is the system state; $u$ is the know system input; $y$ is the measured output; and $w$ and $z$ are noise.

Given the discrete linear system above, the $\mathrm{H}$ infinity filter seeks to solve the following:

$$
\min _{\widehat{x}} \max _{w, Z} \mathrm{~J}
$$


where $\mathrm{J}$ is an arbitrary measure of the validity of the $\mathrm{H}$ infinity state estimator. In other words, this problem is saying: given the worst (or max) possible values of $w$ and $z$, we want to find a state estimate that will minimize the worst possible effect that the noise, w and $z$, will have on the estimation error. The above problem statement also gives indication as to why the $\mathrm{H}$ infinity filter is sometimes called the minimax filter.

Given the definition of the discrete linear system along with the problem that the $\mathrm{H}$ infinity filter seeks to solve, one can write the filter equations as follows:

$$
\begin{aligned}
& L_{k}=\left(I-Q P_{k}+C^{T} V^{-1} C P_{k}\right)^{-1} \\
& K_{k}=A P_{k} L_{k} C^{T} V^{-1} \\
& \hat{x}_{k+1}=A \hat{x}_{k}+B u_{k}+K_{k}\left(y_{k}-C \hat{x}_{k}\right) \\
& P_{k+1}=A P_{k} L_{k} A^{T}+W
\end{aligned}
$$

$\mathrm{K}_{\mathrm{k}}$ is the $\mathrm{H}$ infinity gain matrix. The state estimate at $\mathrm{t}=0$ should be initialized to the designer's best guess as to what the initial state of the system is. From this, P should be set small if one is highly confident in the guess chosen for the initial state estimate.

Looking at the $\mathrm{H}$ infinity filter equations, it is reasonable to notice that there are many more filter parameters that require tweaking than can be found in the Kalman filter equations. For this reason, it may be more difficult to fine-tune an $\mathrm{H}$ infinity filter than a Kalman filter. 


\subsection{3 $\alpha-\beta$ Filter}

The $\alpha-\beta$ filter is frequently thought of as one of the simplest tracking filters ${ }^{[12]}$. The $\alpha-\beta$ filter is a steady-state Kalman filter that can be applied to a two-state Newtonian system with an input of position measurements. The $\alpha-\beta$ filter is defined by the following system equations:

$$
\begin{aligned}
& x_{s}(k)=x_{p}(k)+\alpha\left[x_{m}(k)-x_{p}(k)\right] \\
& V_{s}(k)=V_{s}(k-1)+\beta\left[x_{m}(k)-x_{p}(k)\right] / T \\
& x_{p}(k+1)=x_{p}(k)+V_{s}(k) T
\end{aligned}
$$

Where $x_{s}(k)$ is the smoothed position, $V_{s}(k)$ is the smoothed velocity, $x_{p}(k)$ is the predicted position, $x_{m}(k)$ is the measured position, $T$ is the time between positions, and $\alpha$ and $\beta$ are the system gains. Typically, $\alpha$ should be greater than zero and $\beta$ should be less than one.

The values for $\alpha$ and $\beta$ are often determined experimentally. This may seem daunting, but it is quite simple when you start from reasonable values and adjust based on your results. As previously stated, the value for $\beta$ should be small so that speed estimates are not excessively affected by slight variations from the input measurements. A good starting value for $\beta$ is between 0.05 and 0.10 . The $\alpha$ correction is not directly related to the Newtonian kinematic equations; rather it is used to maintain consistency between velocity and acceleration variables. A good initial value range for $\alpha$ is between 0.10 and $0.20^{[6]}$. 


\subsubsection{Particle Filter}

Particle Filters were discovered around the 1940s, however were not widely used until

the 1980 s due to the large amount of computational power required to run the filter ${ }^{[10]}$. The reason for the large computational power is the statistically-intensive, brute-force approach to filtering that works very well for systems that cannot easily be filtered with more standard approaches, such as the Kalman filter. In other words, the particle works very well for systems that are highly nonlinear.

The particle filter relies on a Bayesian approach to state estimation. In fact, the derivation of the particle filter is a probability-based estimator that is based on Bayes' Rule. Bayes' Rule is used to statistically determine both the a priori probability density function ( $p d f)$ and the a posteriori probability density function of output of a given system. These results are then combined recursively to make the particle filter. The following are the steps used to implement the particle filter.

1. System and Measurement Equations are given by:

$$
\begin{aligned}
& x_{k+1}=f_{k}\left(x_{k}, w_{k}\right) \\
& y_{k}=h_{k}\left(x_{k}, v_{k}\right)
\end{aligned}
$$

where $\left\{w_{k}\right\}$ and $\left\{v_{k}\right\}$ are independent white noise processes with known probability density functions, $\left\{\mathrm{x}_{\mathrm{k}}\right\}$ is the known input, and $\left\{\mathrm{y}_{\mathrm{k}}\right\}$ is the output.

2. The user then assumes that the pdf of the intial state $p\left(x_{0}\right)$ is known and randomly generates $\mathrm{N}$ intial particles based on the $\mathrm{pdf} \mathrm{p}\left(\mathrm{x}_{0}\right)$. The parameter 
$\mathrm{N}$ is chosen by the user while considering trade-offs such as computational effort and estimation accuracy.

3. For discrete values of $\mathrm{k}$, that is $\mathrm{k}=1,2, \ldots$, perform the following steps:

(a) Obtain a priori particles $x_{k, i}^{-}$using the known process equation and the known pdf of the process noise given by:

$$
x_{k, i}^{-}=f_{k-1}\left(x_{k-1}^{+}, w_{k-1}^{i}\right) \text { for } i=(1,2, \ldots, N)
$$

Where each $w_{k-1}^{i}$ noise vector is randomly generated based on the known pdf of $w_{k-1}$.

(b) Compute the relative likelihood $\mathrm{q}_{\mathrm{i}}$ of each particle $x_{k, i}^{-}$trained on the measurement $\mathrm{y}_{\mathrm{k}}$. Evaluate the pdf $p\left(y_{k} \mid x_{k, i}^{-}\right)$based on the nonlinear measurement equation and the pdf of the measurement noise.

(c) Perform scaling on the relative likelihoods qi obtained in step (b) using the following equation:

$$
q_{i}=\frac{q_{i}}{\sum_{j=1}^{N} q_{j}}
$$

This ensures the sum of the likelihoods is equal to one.

(d) Generate the set of a posteriori particles $x_{k, i}^{+}$based on the relative likelihoods $q_{i}$. This step is called the resampling step. An illustration of the resampling step is shown below in Figure 2.2. 


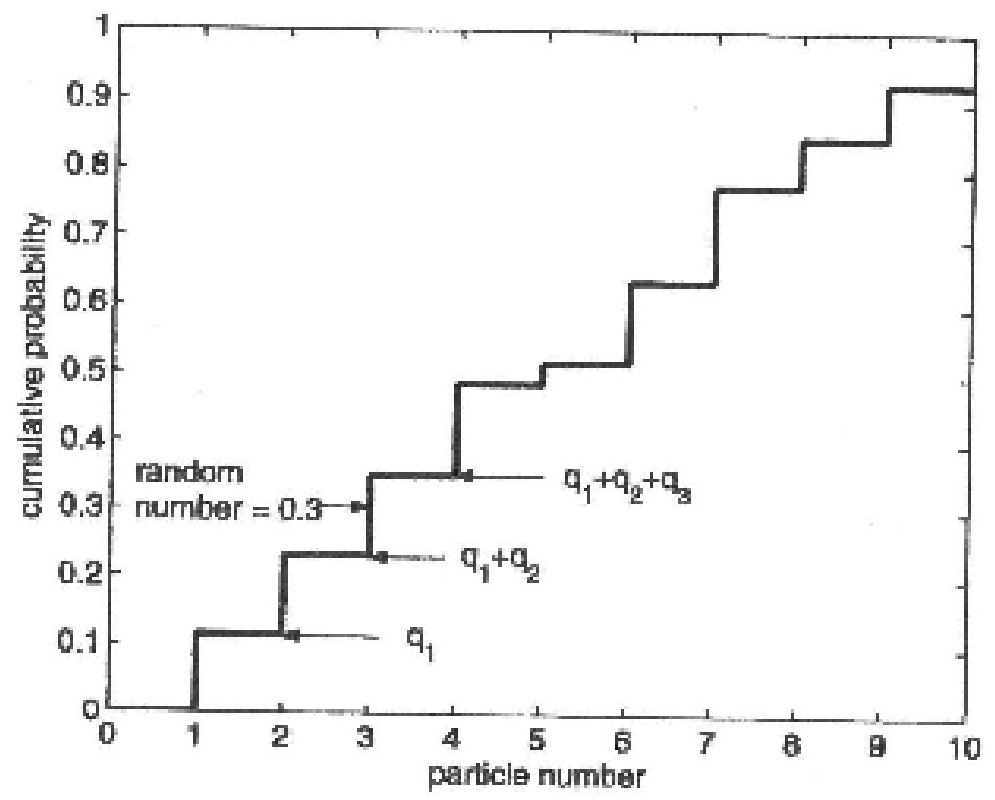

Figure2.2: Example of Resampling in Particle Filter Algorithm

(e) Now there is a set of particles $x_{k, i}^{+}$that are distributed according to the pdf $p\left(x_{k} \mid y_{k}\right)$. For this reason, we can compute any desired statistical measurement of this pdf, however we are typically interested in the mean and covariance.

\subsubsection{Variations of the Kalman Filter}

Several techniques can be preformed given the standard Kalman filter equations which will allow their results to be more accurate in certain situations ${ }^{[10]}$. The alterations render the formulas mathematically equivalent, but will result in filters that improve computational time, precision, and stability. 
The following Figure 2.3 provides an illustration of the variations to the Kalman Filter and a summary of their benefits.

\begin{tabular}{|c|c|c|}
\hline Sequential Kalman Filter & Information Filtering & U-D Filtering \\
\hline Avoids Matrix Inversion & Propagates Inverse of & Increase Precision \\
& Covariance Matrix & \\
\hline
\end{tabular}

Figure 2.3: Variations of the Kalman Filter

\section{Sequential Kalman Filter}

The sequential Kalman filter is a great way to implement the Kalman filter without the need for matrix inversion. This is most useful for use in an embedded system that does not have matrix routines. However, there is a limited use for this filter. It is a requirement that the $R_{k}$ (measurement covariance matrix) is either diagonal or constant. To illustrate the concept of the sequential Kalman Filter, two images are shown in Figures 2.4 and 2.5 . 


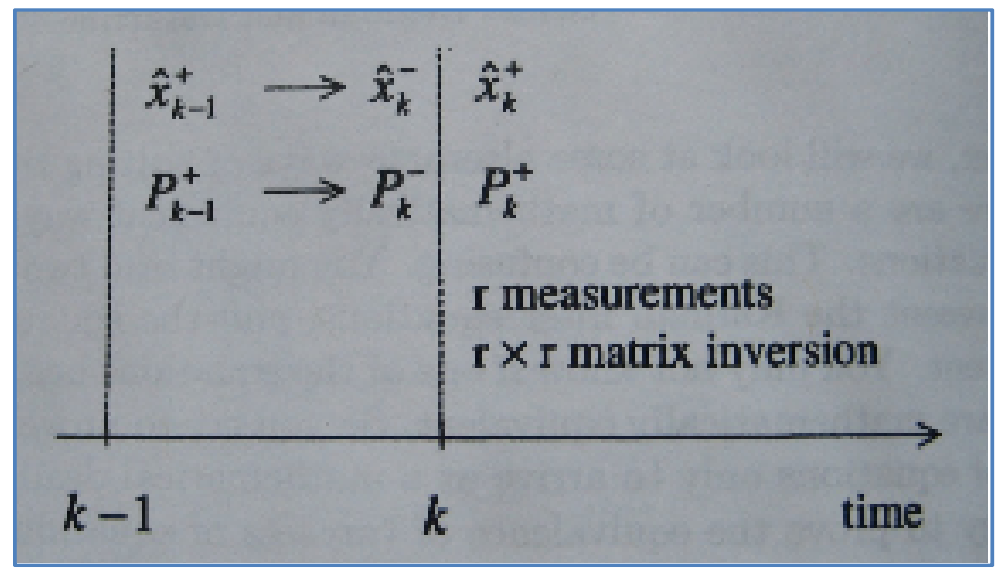

Figure2.4: Measurement update equation of the standard Kalman filter showing $\mathrm{r} x \mathrm{r}$ matrix inversion

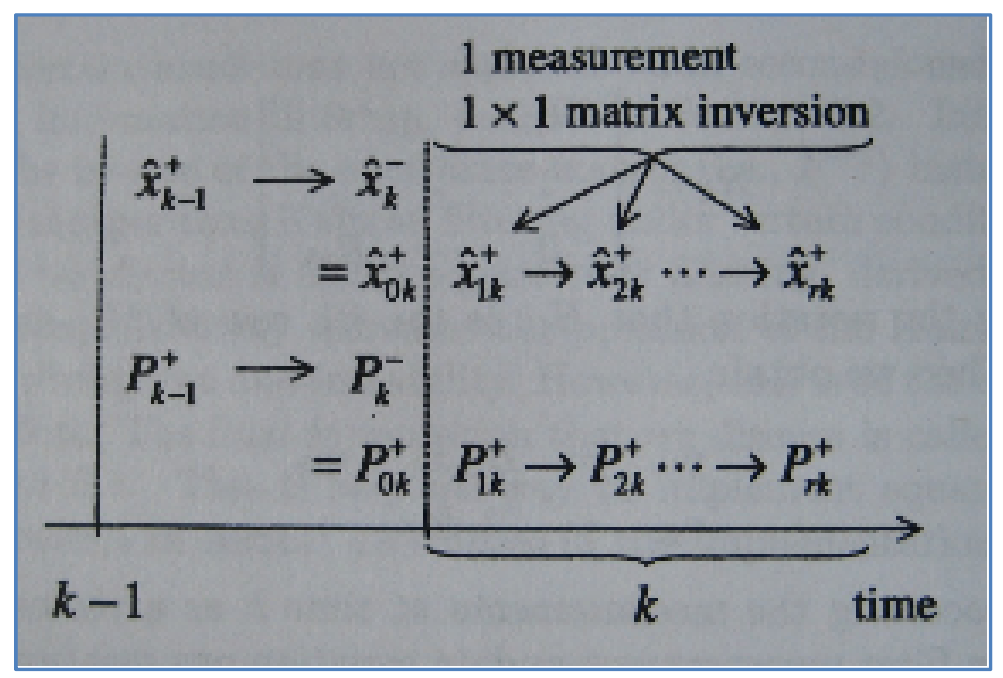

Figure 2.5: Measurement update equation of sequential Kalman filter showing $r$ scalar divisions

The only differences in the equations can be summed up as follows. The gain and covariance updates are obtained from the normal Kalman filter measurement-update equations, however they are simply modified to only apply for scalar conditions.

For $\mathrm{i}=1, \ldots, \mathrm{r}$ : 


$$
\begin{aligned}
& K_{i k}=P_{i-1, k}^{+} H_{i k}^{T}\left(H_{i k} P_{i-1, k}^{+} H_{i k}^{T}+R_{i k}\right)^{-1} \\
& \hat{x}_{i k}^{+}=\hat{x}_{i-1, k}^{+}+K_{i k}\left(y_{i k}-H_{i k} \hat{x}_{i-1, k}^{+}\right) \\
& P_{i k}^{+}=\left(I-K_{i k} H_{i k}\right) P_{i-1, k}^{+}
\end{aligned}
$$

In summary, it only makes sense to use the sequential Kalman filter if one does not have access to matrix inversion routines. Otherwise, this method requires far more calculations than the standard Kalman filter.

\section{Information Filtering}

This implementation of the Kalman filter propagates the inverse of the P matrix rather than propagating $\mathrm{P}^{[10]}$. Recall that $\mathrm{P}$ represents the uncertainty in the state estimate. If $P$ is large, this implies there is a lot of error in the state estimate. To remedy for this situation, a new matrix, called the Information matrix, is created and defined as follows:

$$
I=P^{-1}
$$

where I now represents the certainty (as opposed to uncertainty) in the state estimate. The information filter is given by the following equations 


$$
\begin{aligned}
& I_{k}^{-}=Q_{k-1}^{-1}-Q_{k-1}^{-1} F_{k-1}\left(I_{k-1}^{+}+F_{k-1}^{T} Q_{k-1}^{-1} F_{k-1}\right)^{-1} F_{k-1}^{T} Q_{k-1}^{-1} \\
& I_{k}^{+}=I_{k}^{-}+H_{k}^{T} R_{k}^{-1} H_{k} \\
& K_{k}=\left(I_{k}^{+}\right)^{-1} H_{k}^{T} R_{k}^{-1} \\
& \hat{x}_{k}^{-}=F_{k-1} \hat{x}_{k-1}^{+}+G_{k-1} u_{k-1}
\end{aligned}
$$

In the standard Kalman filter, there is an inversion required with an $r \times r$ matrix where $r$ is the number of measurements. The information filter requires several $n \times n$ matrix inversions, where $n$ is the number of states. If it is the case that $r \gg n$ (many more measurements than states), then it may be computationally more effective to use the information filter.

By using the information filter, if the initial uncertainty is infinite, we cannot numerically set the P matrix to infinity; however we can numerically set the I matrix to zero. This makes the filter mathematically more precise for the zero initial certainty situation. The Kalman filter is more precise for the zero initial uncertainty situation.

\section{U-D Filtering}

The U-D filter was derived to increase the numerical precision of the Kalman filter. As a tradeoff, the computational cost of the filter increases. The premise behind the U-D filter is to factor the $n \times n$ matrix $\mathrm{P}$ as $\mathrm{UDU}^{\top}$, where $\mathrm{U}$ is an $n \times n$ upper triangular matrix with ones along its diagonal and $\mathrm{D}$ is an $n \times n$ diagonal matrix. The $U-D$ filtering algorithm can be summarized as follows:

1. Start with the a priori estimation covariance $P^{-}$at time k. Define $P_{0}=P^{-}$. 
2. For $i=1, \ldots, r$, where $r$ is the number of measurements, perform the following:

(a) Define $\mathrm{H}_{i}$ as the ith row of $H, R_{i}$ as the ith diagonal entry of T. Also define the constant $\alpha_{i}=H_{i} P_{i-1} H_{i}^{T}+R_{i}$

(b) Perform a U-D factorization of $\mathrm{P}_{\mathrm{i}-1}$ to obtain $\mathrm{U}_{\mathrm{i}-1}$ and $\mathrm{D}_{\mathrm{i}-1}$, and then form the matrix UDU ${ }^{\top}$ using the following equations:
a. $\bar{U} \bar{D} \bar{U}^{T}=\left[D_{i-1}-\frac{1}{\alpha_{i}}\left(D_{i-1} U_{i-1}^{T} H_{i}^{T}\right)\left(D_{i-i} U_{i-1}^{T} H_{i}^{T}\right)^{T}\right]$
b. $U_{i} D_{i} U_{i}^{T}=\left(U_{i-1} \bar{U}\right) \bar{D}\left(U_{i-1} \bar{U}\right)^{T}$

(c) Compute $U_{i}$ and $D_{i}$ as follows:
a. $U_{i}=U_{i-1} \bar{U}$
b. $D_{i}=\bar{D}$

3. The a posterirori estimation covariance is given as $\mathrm{P}^{+}=\mathrm{U}_{\mathrm{r}} \mathrm{D}_{\mathrm{r}} \mathrm{U}_{\mathrm{r}}^{\mathrm{T}}$.

The U-D filter results in twice as much precision as the standard Kalman filter [10]. 


\section{Extended Target Simulation Design and Results}

\subsection{Generating MSTAR Images to Use for Simulation}

The images created for simulation feature several frames of the same target at multiple $X-Y$ coordinate locations as the target moves throughout space. Each image is 200 by 200 pixels and contains a target that is approximately 50 pixels by 30 pixels.

The images are comprised of random noisy background images generated from true MSTAR image noise. On top of this background, a target generated from true MSTAR images is placed. This target is rotated between -10 and +10 degrees to simulate actual driving maneuvers. In addition to the noise that is present in the true MSTAR images, additional noise is added in MATLAB in the following forms: salt and pepper, speckle, and Gaussian. Figure 3.1 shows an example of how a random MSTAR image is created. The following sections discuss each MATLAB function in greater detail.

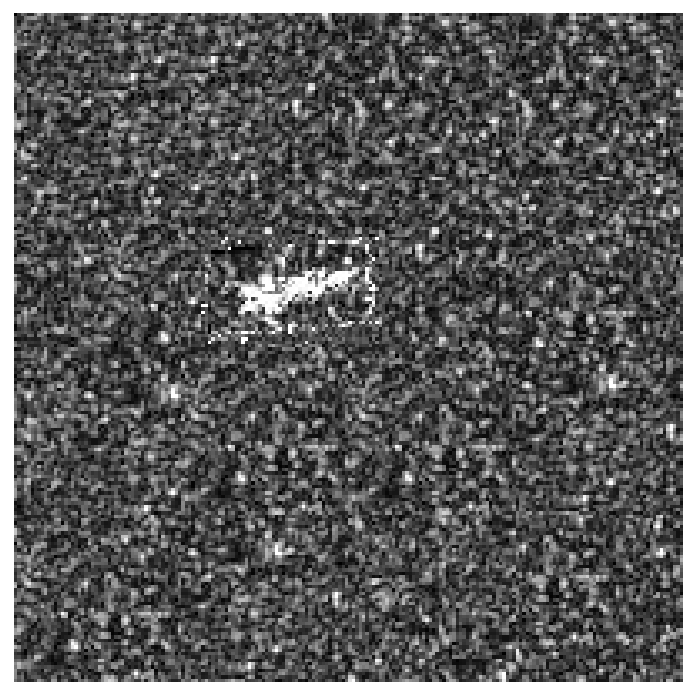

Figure 3.1: Sample MSTAR image including random background and rotated target 


\subsection{Generating Random Noisy Images}

To better simulate real MSTAR SAR images, images with random noise were created. There are several ways to do this, however the way I chose resulted in the most realistic noise best suited for my simulations. Processed MSTAR images from Sandia Labs ${ }^{[3]}$ were used to extract twenty unique 50 by 50 pixel images of true MSTAR background noise.

genNoise.m: This MATLAB function processed the 50 by 50 pixel images of true MSTAR background noise using the following steps:

- Generate a blank 200 by 200 pixel image that will eventually become the background random noise used for simulation.

- Generate sixteen random numbers between one and twenty to be used to randomly choose which of the twenty unique MSTAR background noise images to add to the blank 200 by 200 pixel blank image.

- Generate a tiled matrix of random unique MSTAR background image noises. In any given 200 by 200 pixel blank image, there will be sixteen randomly generated 50 by 50 pixel images of true MSTAR background noise

- Repeat this process until twenty-six 200 by 200 pixel images are created

Figure 3.2 shows a sample image created, first by showing the sixteen images that will be tiled together, and then showing the resulting image they create. 


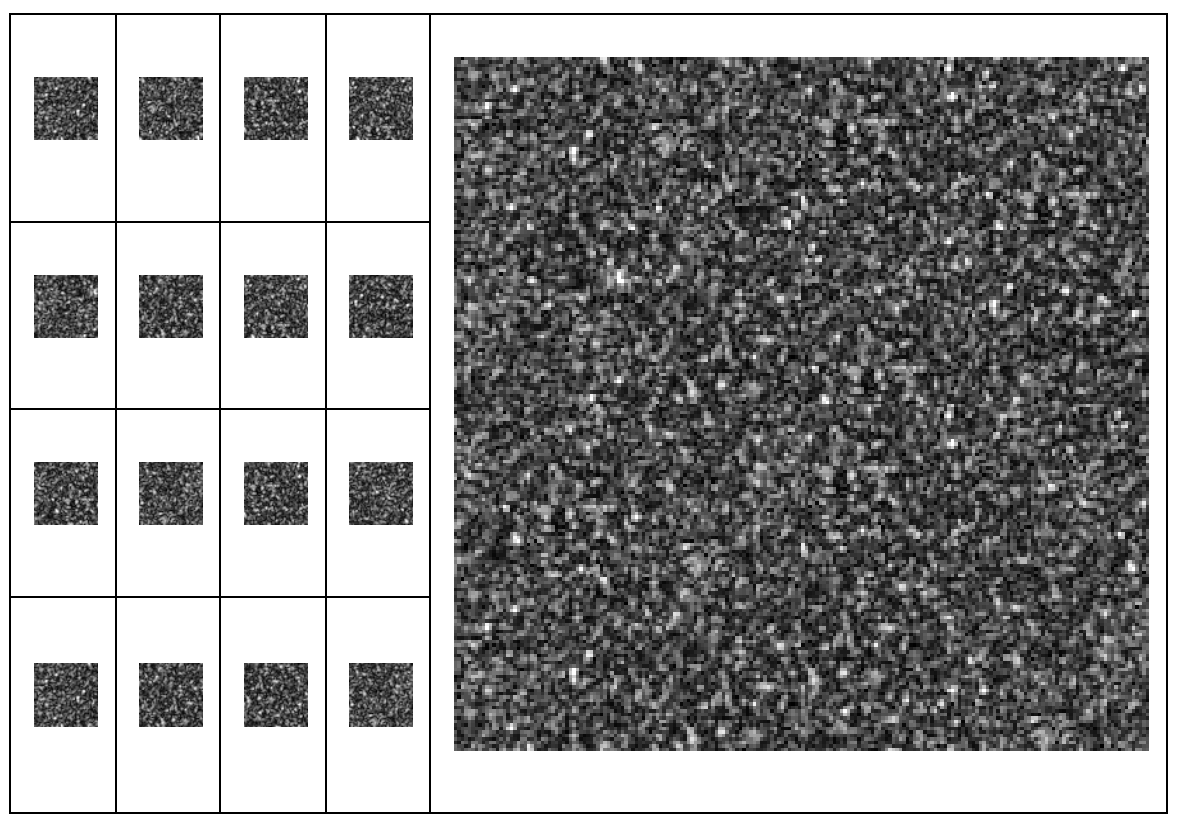

Figure 3.2: Sample Noisy Background Image generated using genNoise.m

\subsection{Generating Rotated Target Images}

Instead of simply placing the target image onto each background image generated in genNoise.m, it was decided that a more realistic approach is to insert images of the target that are rotated slightly. This provides a more accurate image for simulation because it accounts for a truer maneuver of a vehicular target.

The MATLAB function rotateImage. $m$ takes an image and the corresponding angle to which one wishes to rotate the image. For the purpose of generating rotated targets, the same target was used while the angle varied between -10 and +10 degrees. Using the cosine and sine functions, new $\mathrm{X}$ and $\mathrm{Y}$ coordinates are determined for all pixels in the original image. MATLAB then utilizes its function 'imrotate ( )' to rotate the image. Samples of the target are shown rotated between the range of -10 to +10 degrees in Figure 3.3. 


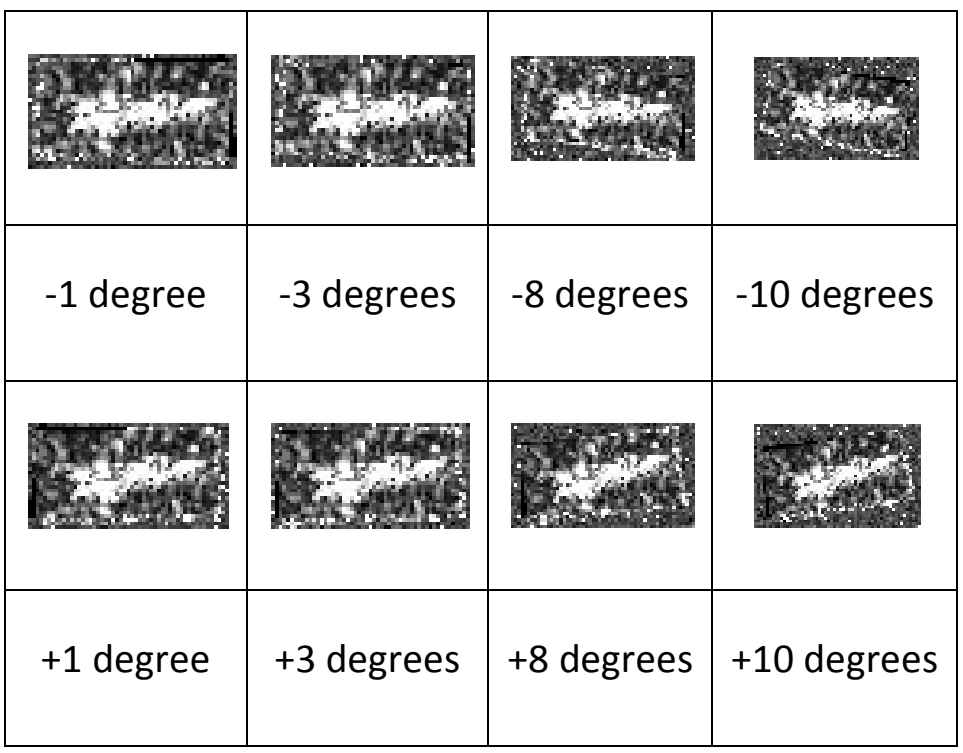

Figure 3.3: Sample targets rotated between -10 degrees and +10 degrees

\subsection{Post-Processing Filtering of MSTAR Images}

Post-processing of MSTAR images is required because the images are inherently noisy.

This processing is necessary to make the Kalman filter run more accurately, since performing the same filtration techniques on every image will allow for a common metric. The following are the post-processing steps taken in the program findBlob.m:

- Threshold the original image to create a binary image. This threshold carries a value between 0 and 1 . The output binary image will have a value of 1 (white) for all pixels in the input image with a brightness greater than the threshold and will have a value of 0 (black) for all pixels less than or equal to the threshold. findBlob.m uses a threshold of 0.5. Figure 3.4 shows an example of an original MSTAR image and the results of the threshold. 


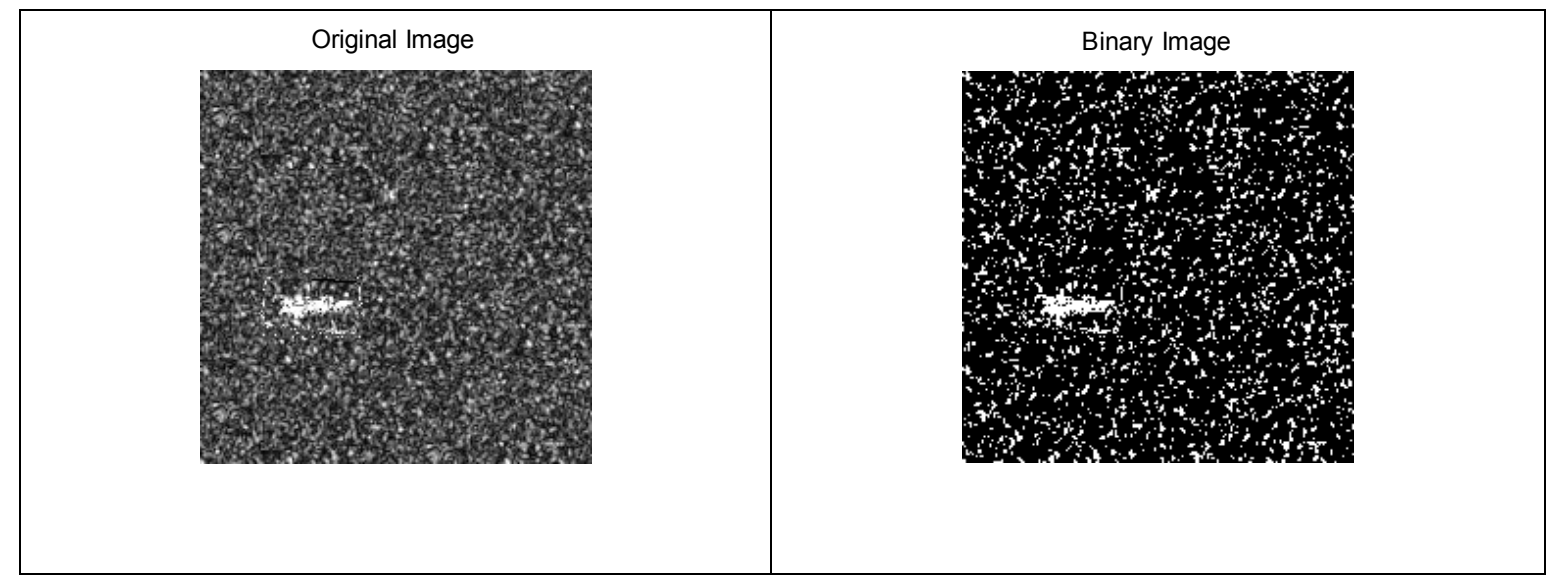

Figure 3.4 - Threshold Step of Post-Processing

- The function bwlabel is used to label the binary image shown in Figure 3.4.

Bwlabel returns a matrix containing labels for all connected components that are connected by 4 or more pixels.

- Once the image is labeled, the blob with the maximum area is located and everything else in the image is filtered out, leaving only the largest blob-which should correspond to the target. This yields a fully-filtered image as shown in Figure 3.5.

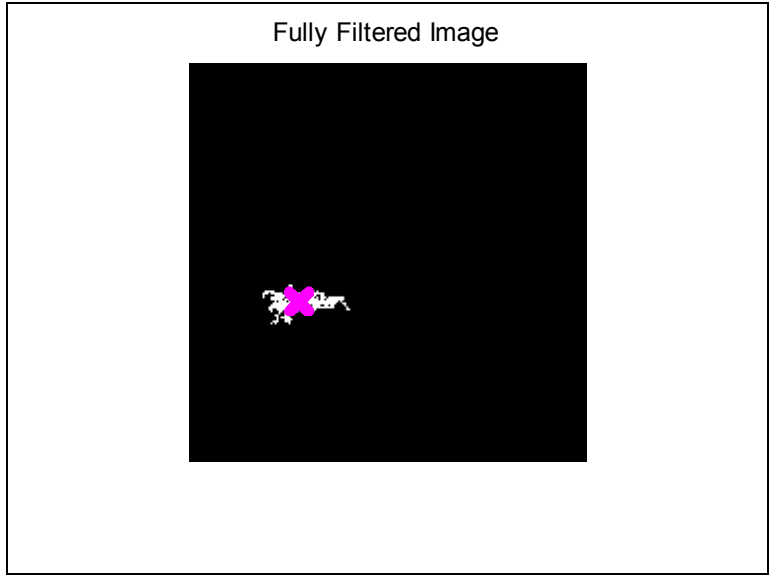

Figure 3.5 - Fully-Filtered Image of Target showing an ' $x$ ' where the centroid is located 
The next step is to determine the centroid of the fully-filtered target shown in Figure 3.5. The centroid consists of the row and column pixel locations of the center of the blob.

\subsection{Kalman Filtering of MSTAR Images}

The Kalman filter algorithm used to process the MSTAR images follows the process for 2-D Kalman filtering introduced in Chapter 2. The program myKa lman . $m$ first initializes all of the Kalman filter parameters. Next, each image is looped through and is passed to findBlob. m to determine its centroid location. This centroid location is then used as the current input state to the Kalman filter which then updates all parameters. The true centroid locations (as determined in findBlob.m) are plotted with the Kalman centroid locations (as determined in myKalman.m) and displayed, giving the user a visual representation of the accuracy of the Kalman filter algorithm. A sample Kalman filter image is shown in Figure 3.6. The smaller, red ' $x$ ' indicates the Kalman estimated state, whereas the larger, magenta ' $x$ ' represents the true centroid location.

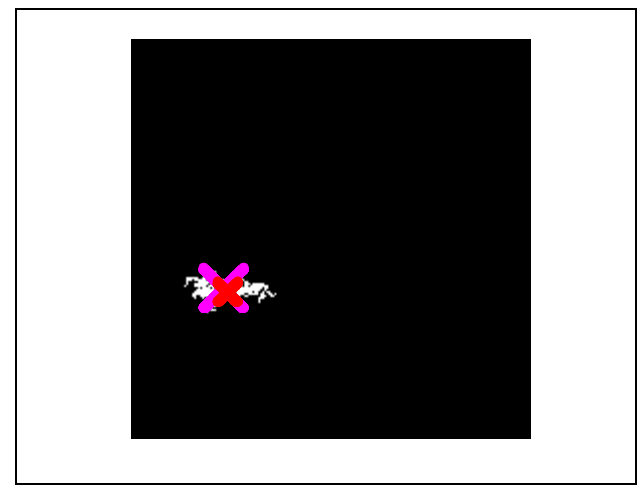

Figure 3.6 - Sample Kalman Algorithm Image Showing True and Kalman Estimated State 


\subsection{Extended Target Simulation Results}

Using the same set of 26 extended target images, both the Kalman filter and $\mathrm{H}$ Infinity

filter were tested for performance. Using conservative, un-tuned matrix values for $P$ and $Q$ of $P=100$ and $Q=0.01$, the results for both filters appear to be very similar. The results for the Kalman filter are shown in Figure 3.7, while the results for the $\mathrm{H}$ Infinity filter are shown in Figure 3.8.

When altering the values of $P$ or $Q$, it was noticed that there were certain values of $P$ or Q that would yield undesirable results, such as unstable filters, or poorly tuned filters that take too long to converge. This phenomenon applies to both filters, however not for the same values of $\mathrm{P}$ or $\mathrm{Q}$. For this reason, a Monte Carlo analysis was performed and is discussed in greater detail in the following section. 


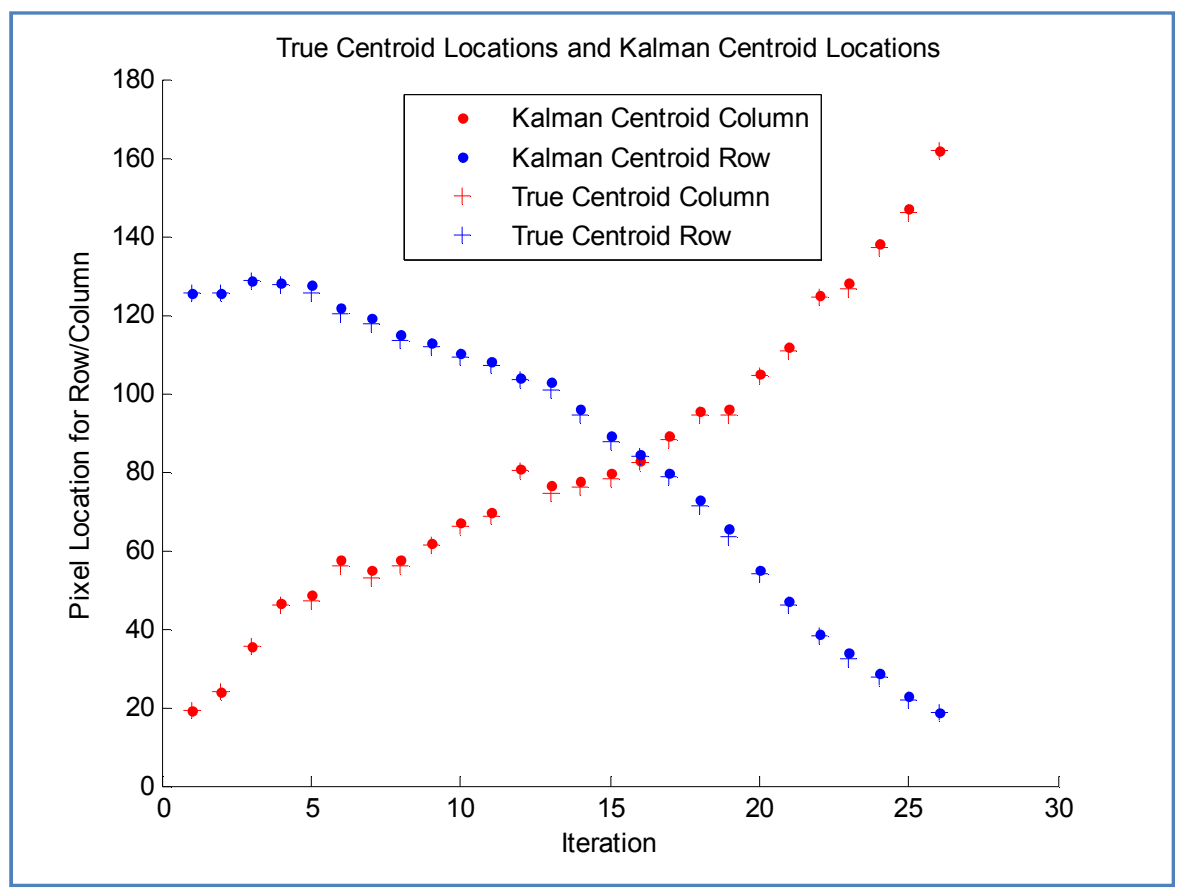

Figure 3.7: True centroid locations and Kalman centroid locations

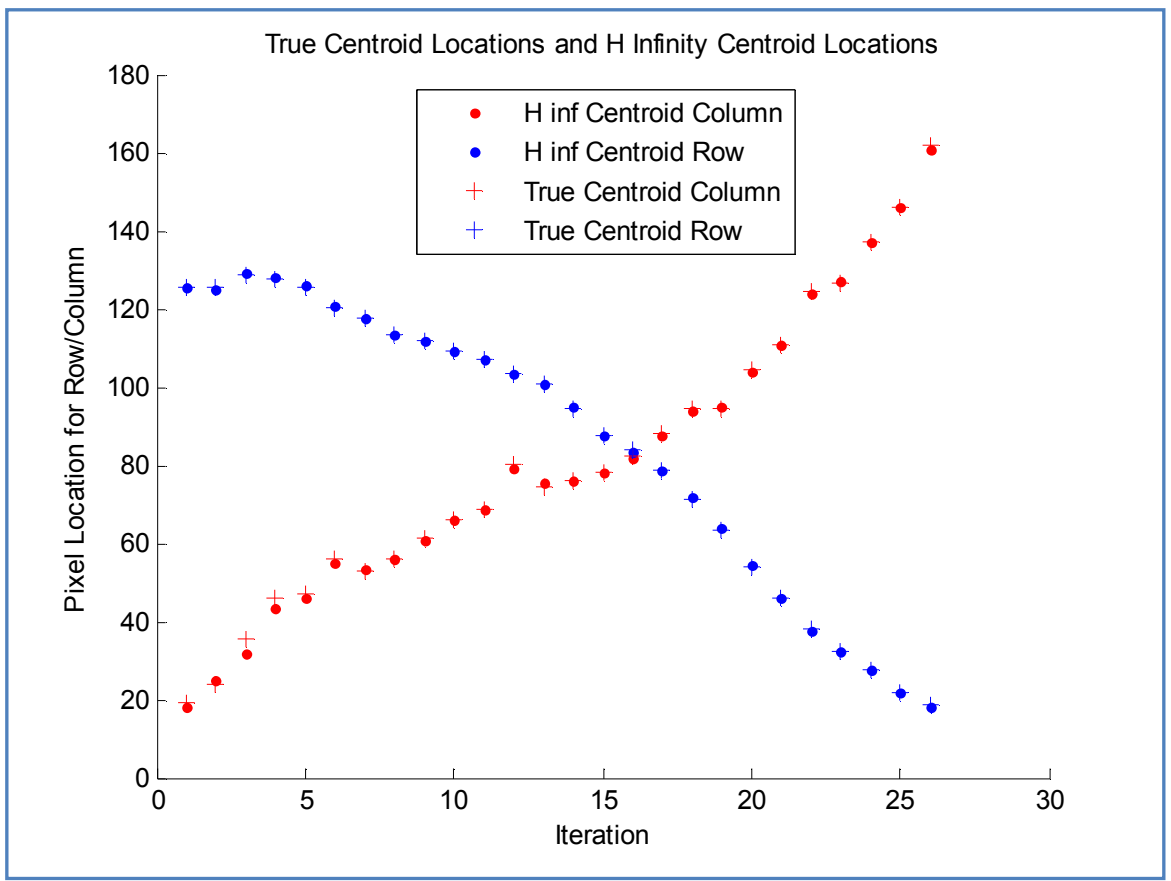

Figure 3.8: True centroid locations and $\mathrm{H}$ Infinity centroid locations 


\subsection{Monte Carlo Analysis}

In both Kalman and $\mathrm{H}$ infinity filtering, the $\mathrm{P}$ (the covariance matrix of the error in the state estimate) and $Q$ (the covariance matrix of the error in the process noise) matrices have a significant impact on the performance of the filter. For this reason, it was advantageous to perform a Monte Carlo analysis on these two matrices.

Monte Carlo analysis refers to a computational algorithm that allows the user to simulate a mathematical system ${ }^{[14]}$. There is no "one" way to perform the analysis, rather there is a typical approach. This typical approach, and the one used for this project, is:

1. Define a domain of inputs

2. Generate inputs from the domain and perform necessary calculations on them

3. Combine the results of the individual calculations into the final result

A MATLAB function was written to step through various values of the $P$ and $Q$ matrices. The following sections illustrate how Monte Carlo Analysis was used to further optimize the original designs of the Kalman and $\mathrm{H}$ Infinity filters. 
3.7.1 Monte Carlo Analysis of Kalman Filter

\begin{tabular}{|c|c|c|c|}
\hline Kalman Filter & Initial Value & Final Value & Increment Value \\
\hline P & 5 & 122.5 & 2.5 \\
\hline Q & 0.0001 & 0.9783 & 0.0208125 \\
\hline
\end{tabular}

Figure 3.9: $P$ and $Q$ Values for Monte Carlo Analysis using the Kalman filter

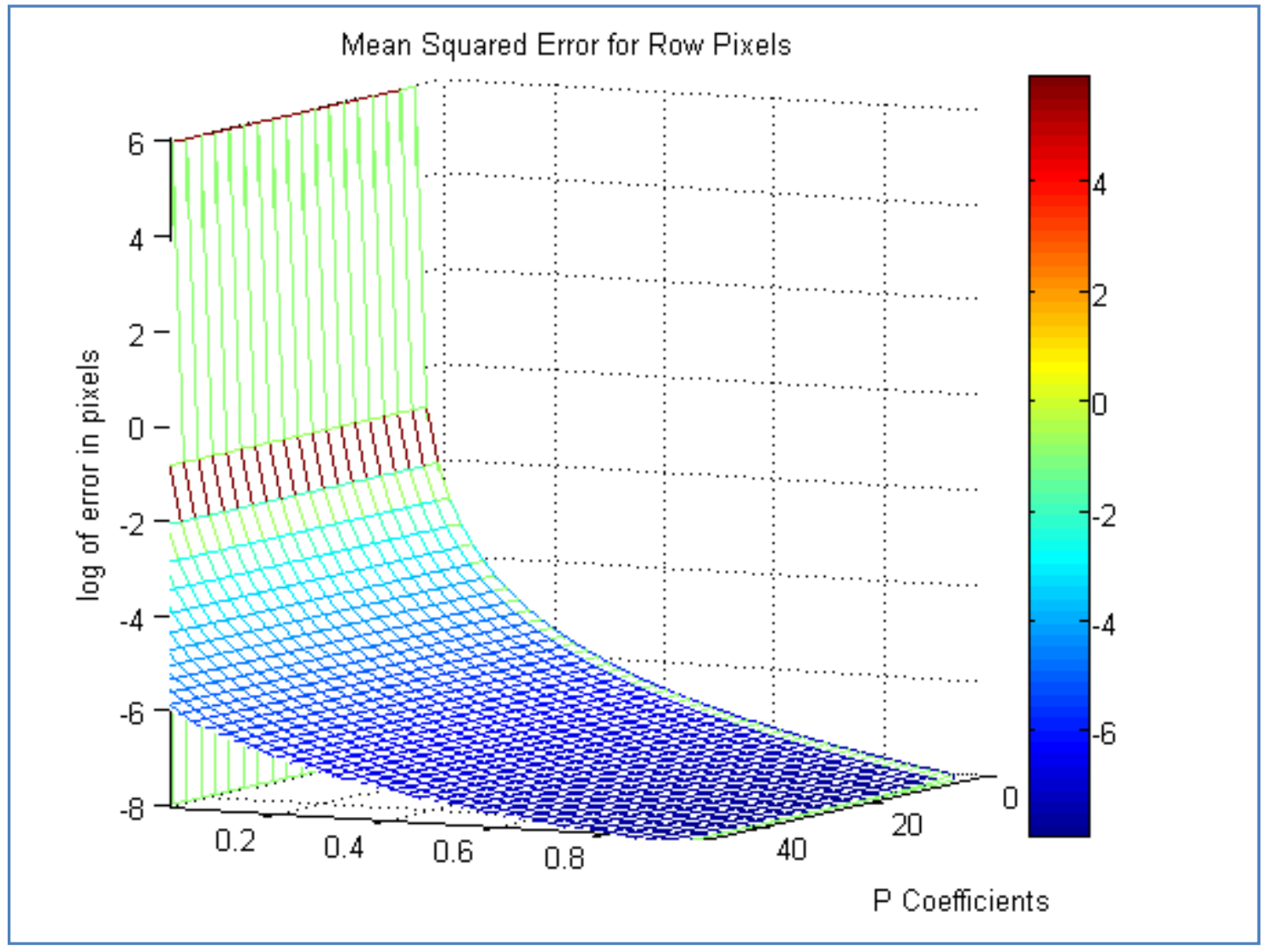

Figure 3.10: Mean-Squared Errors for Row Pixels 


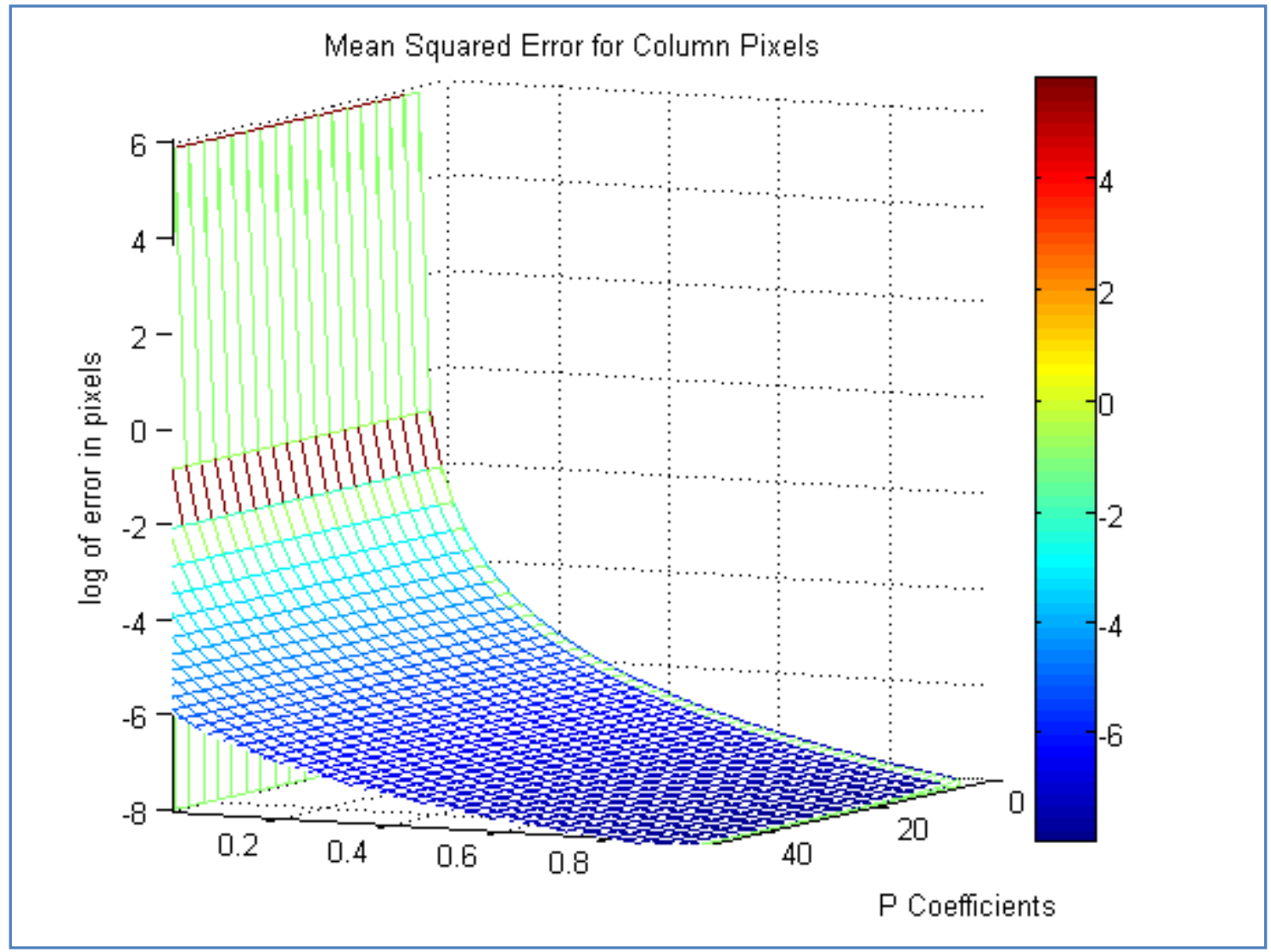

Figure 3.11: Mean-Squared Errors for Column Pixels

The results for the Kalman Monte Carlo analysis indicated that the value of the $\mathrm{P}$ coefficient has no effect on the mean-squared error for a given $Q$ value. 
3.7.2 Monte Carlo Analysis of H Infinity Filter

\begin{tabular}{|c|c|c|c|}
\hline H Infinity Filter & Initial Value & Final Value & Increment Value \\
\hline P & 5 & 154 & 1 \\
\hline Q & 0.001 & 0.2245 & 0.0015 \\
\hline
\end{tabular}

Figure 3.12: Monte Carlo Analysis Parameters for $\mathrm{H}$ Infinity Filter

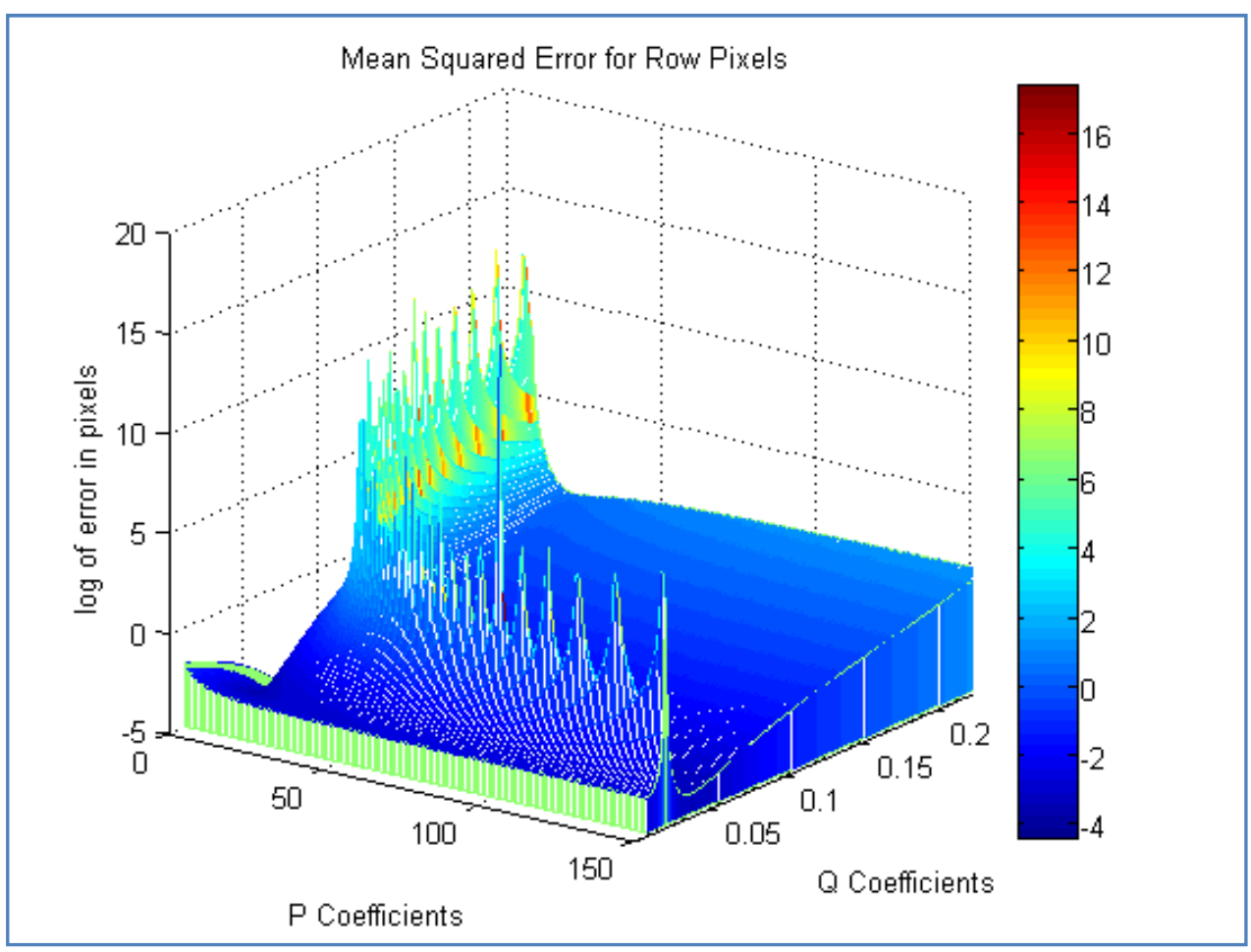

Figure 3.13: Mean-Squared Error for Row Pixels with H Infinity Filter 


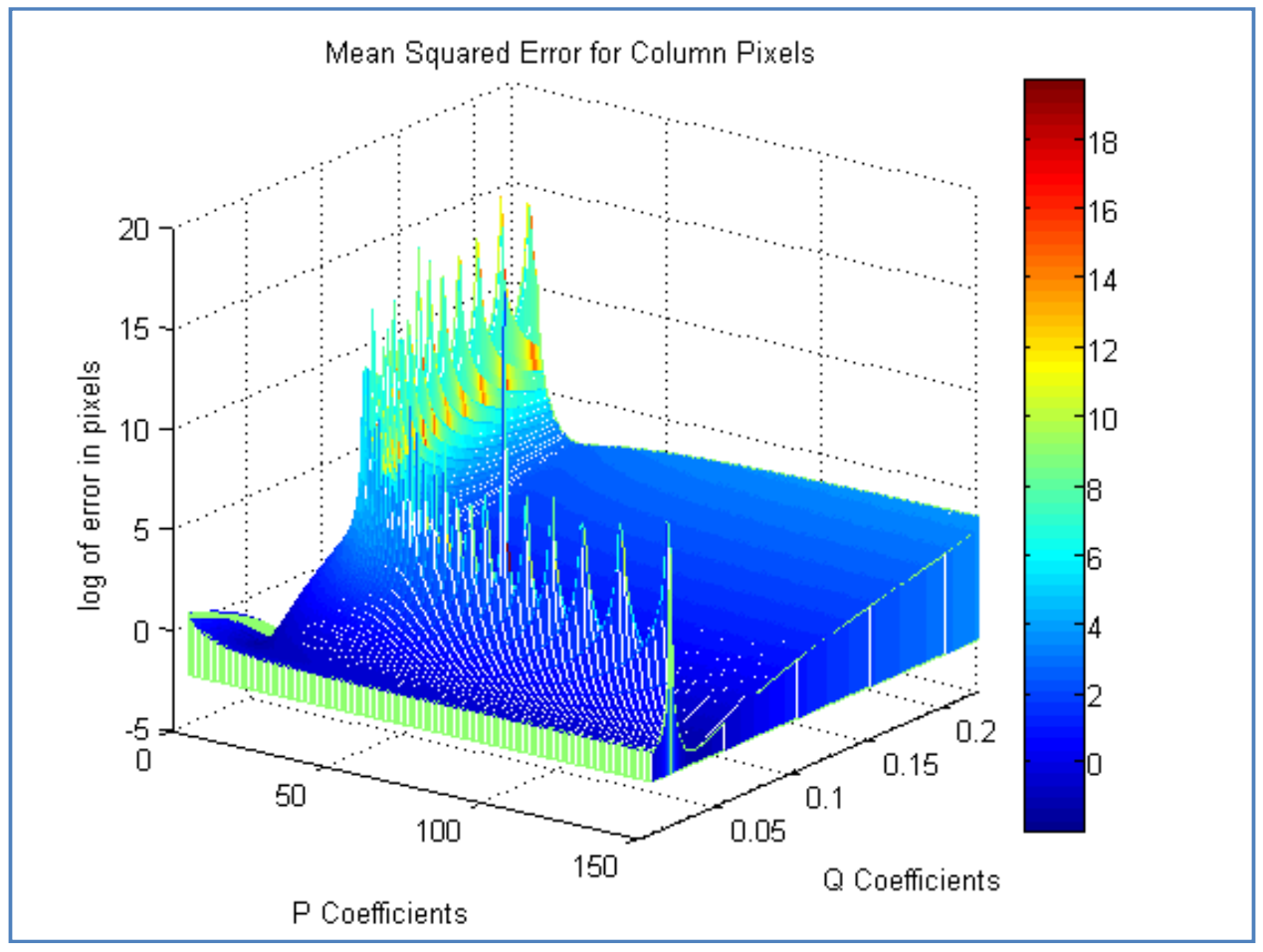

Figure 3.14: Mean-Squared Error for Column Pixels with H Infinity Filter

It is important to note that in the results of the Monte Carlo analysis, it may appear that the filter goes unstable at times (judging by the very large mean-squared error for certain values of $P$ and $Q$ ). This is not the case, however. After only a few iterations, the filter quickly stabilizes and continues to track as expected. The results can be seen in Figure 3.15 for the $H$ Infinity filter when $P=11$ and $Q=0.166$. 


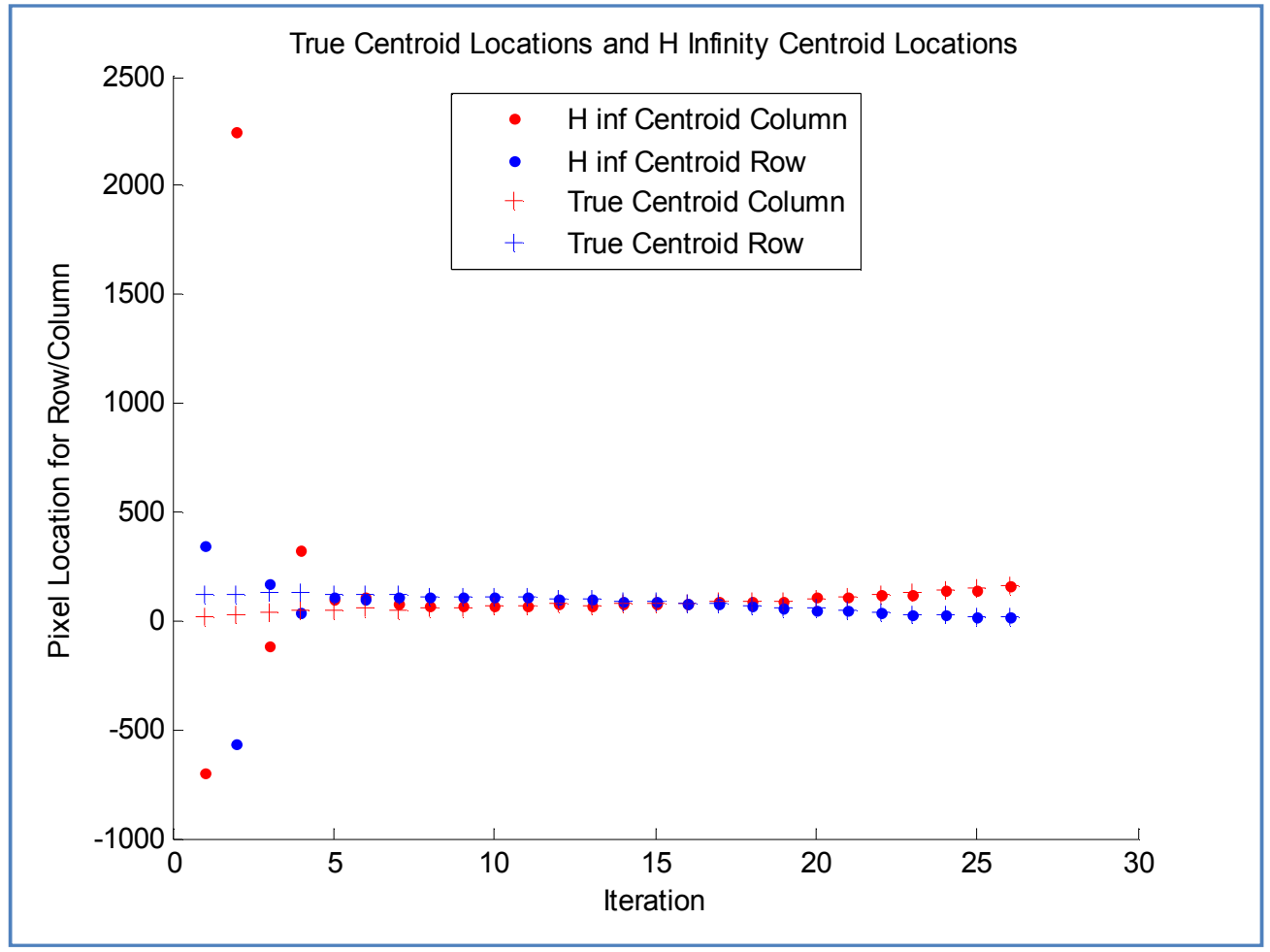

Figure 3.15: Sample of large mean-squared error graphical results for $\mathrm{H}$ Infinity filter 


\section{Point Target Simulation Design and Results}

\subsection{Point Target Discussion}

While the chapter on extended targets is useful to illustrate basic target tracking principles, it is not an entirely accurate representation of true target tracking processes. This chapter serves the purpose of expanding the research performed in Chapter 3 on extended targets and focusing on point targets.

With extended targets, it is fairly easy to recognize a target in a given image as it encompasses many pixels and is often vastly different in intensity and size from the background noise. However, as the detector gets farther away from a target, the target will begin to appear as a smaller blob in the image. Beyond a certain distance away, a target's location may be uncertain as it will only encompass one pixel in the entire image ${ }^{[4]}$. Because of the target's small size, both detection and tracking pose difficulties.

This chapter on point targets will illustrate the need for an alternate form of tracking besides the traditional Kalman and $\mathrm{H}$-Infinity tracking presented in Chapter 3. To illustrate the limitations to the Kalman and $\mathrm{H}$-Infinity tracking methods, a small circular target present in a noisy image will be analyzed as it becomes progressively smaller. Next, various methods of target tracking of a true point target of 1 pixel will be introduced including a simulation of one of the methods. 


\subsection{Modifications to Original Kalman and H Infinity Functions}

This section focuses on the slight modifications made to the Kalman and H-Infinity functions written for extended targets in Chapter 3. The main difference between the extended target tracking and the circular target tracking is the creation of test images. First, a path for the object is defined. This path represents the centroid of the circle as it traverses the test image. However, a path error is also defined which allows the centroid to be a certain pixel distance away from the true path based on a random decision function. In addition, the type and amount of background noise can be chosen by the user. Next, a circle of specified radius is placed on the path and its position is altered if there is a path error defined for any of its locations. A sample of a test image is shown in Figure 4.1 with a circle of radius 4 .

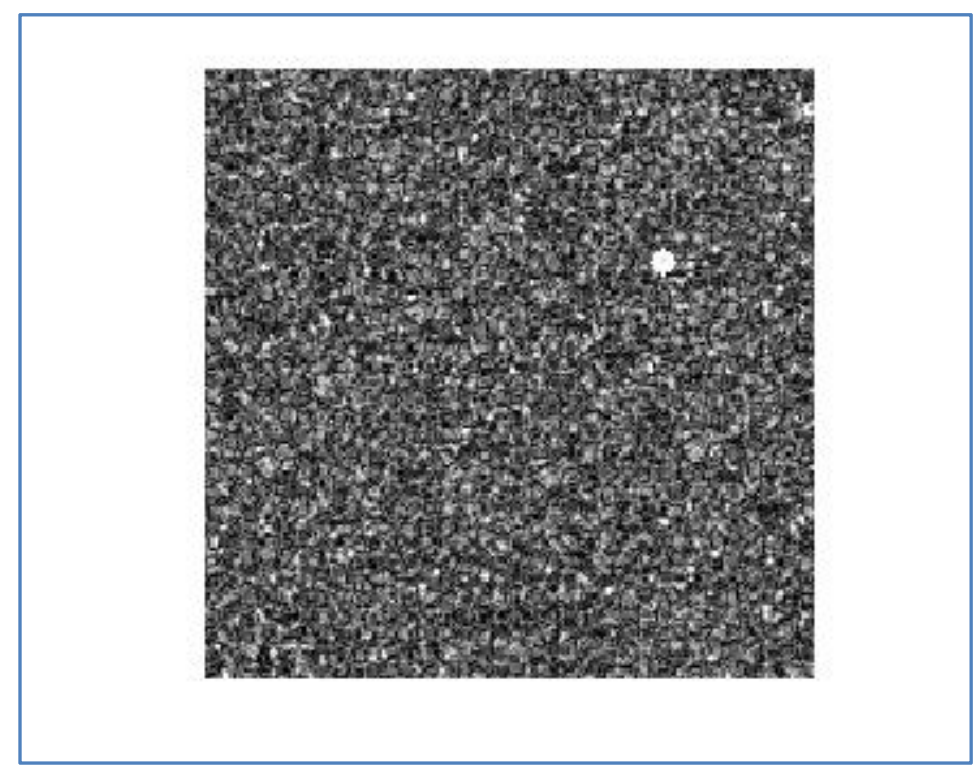

Figure 4.1: Sample Test Image for Circular Target of Radius 4 Pixels 
Next the set of sample test images created along the path is sent through the same Kalman and $\mathrm{H}$-Infinity algorithms as used for the extended target. The above process is repeated for a square target to compare outcomes. A sample test image for a square target is shown in Figure 4.2. The simulation results are plotted and analyzed in the following section.

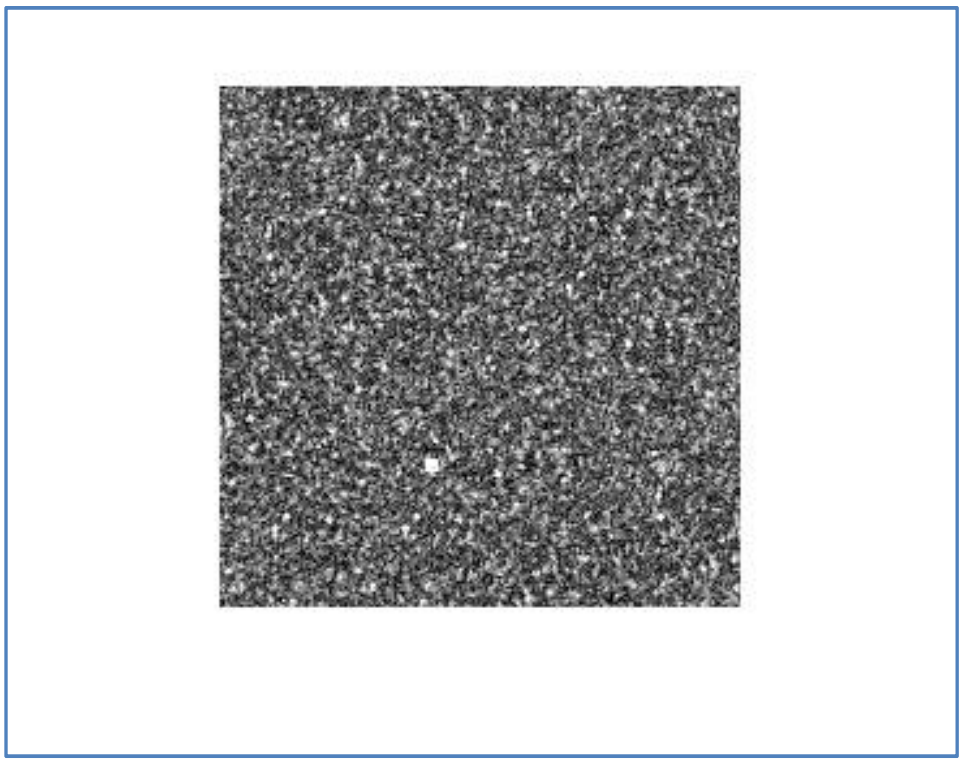

Figure 4.2: Sample Test Image for Square Target of Length 4 Pixels

\subsection{Kalman and H-Infinity Filter Results for Circular and Square Targets}

Perhaps the most obvious finding is that the circular target often becomes lost in the background noise and is sometimes even found to be in an incorrect location. The following image in Figure 4.3 shows a case where the background noise is too prominent and the $\mathrm{f}$ indBlob. m function cannot correctly locate the target. 


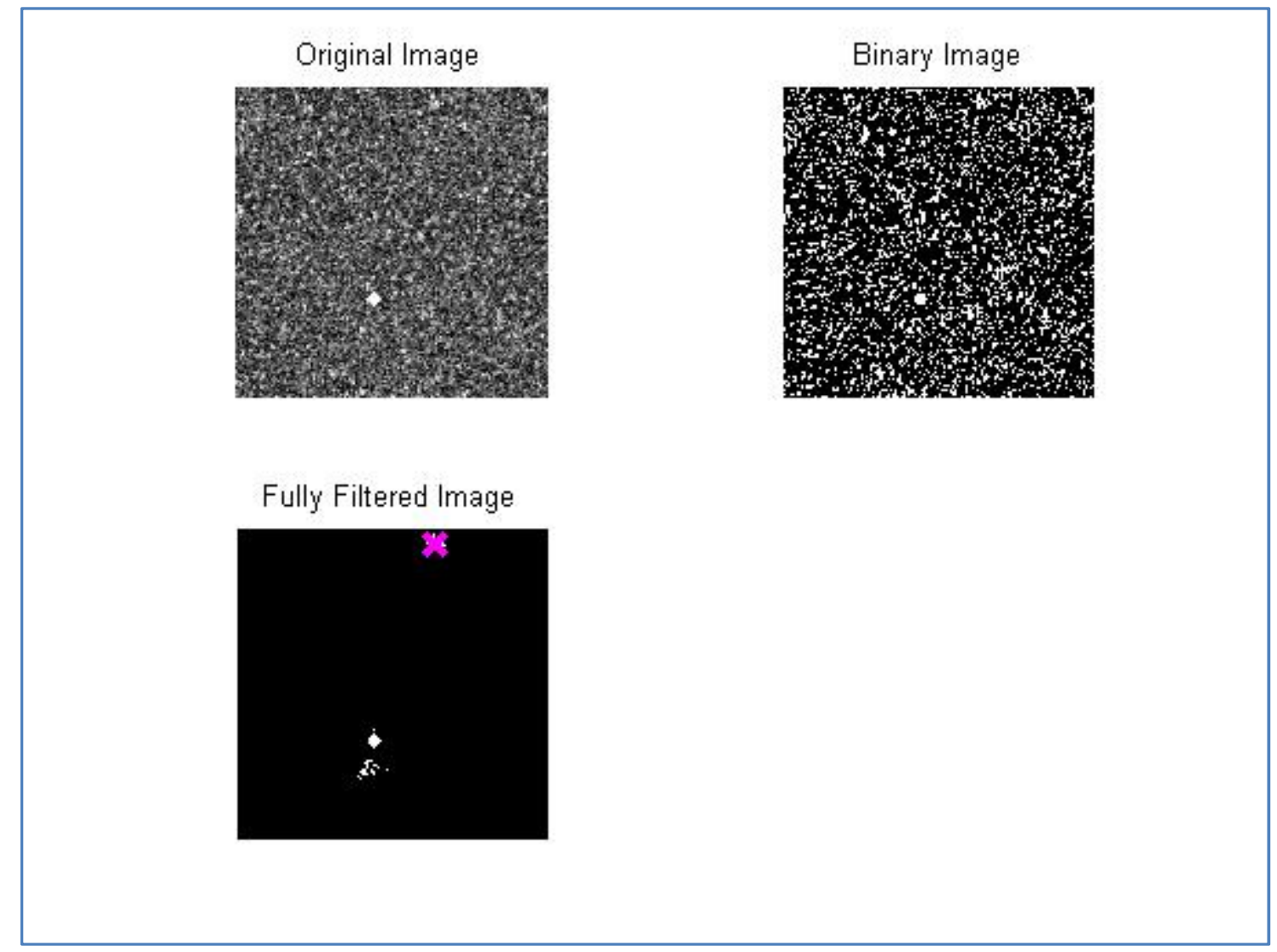

Figure 4.3: Sample Image Showing Incorrectly Located Circular Target Due to Background Noise

When the target is incorrectly located, this causes the filter to go awry for a few iterations; however it can be seen in Figure 4.4 that the Kalman filter very quickly stabilizes back to its original path. However, this is not the case with the $\mathrm{H}$ Infinity filter as shown in Figure 4.5. 


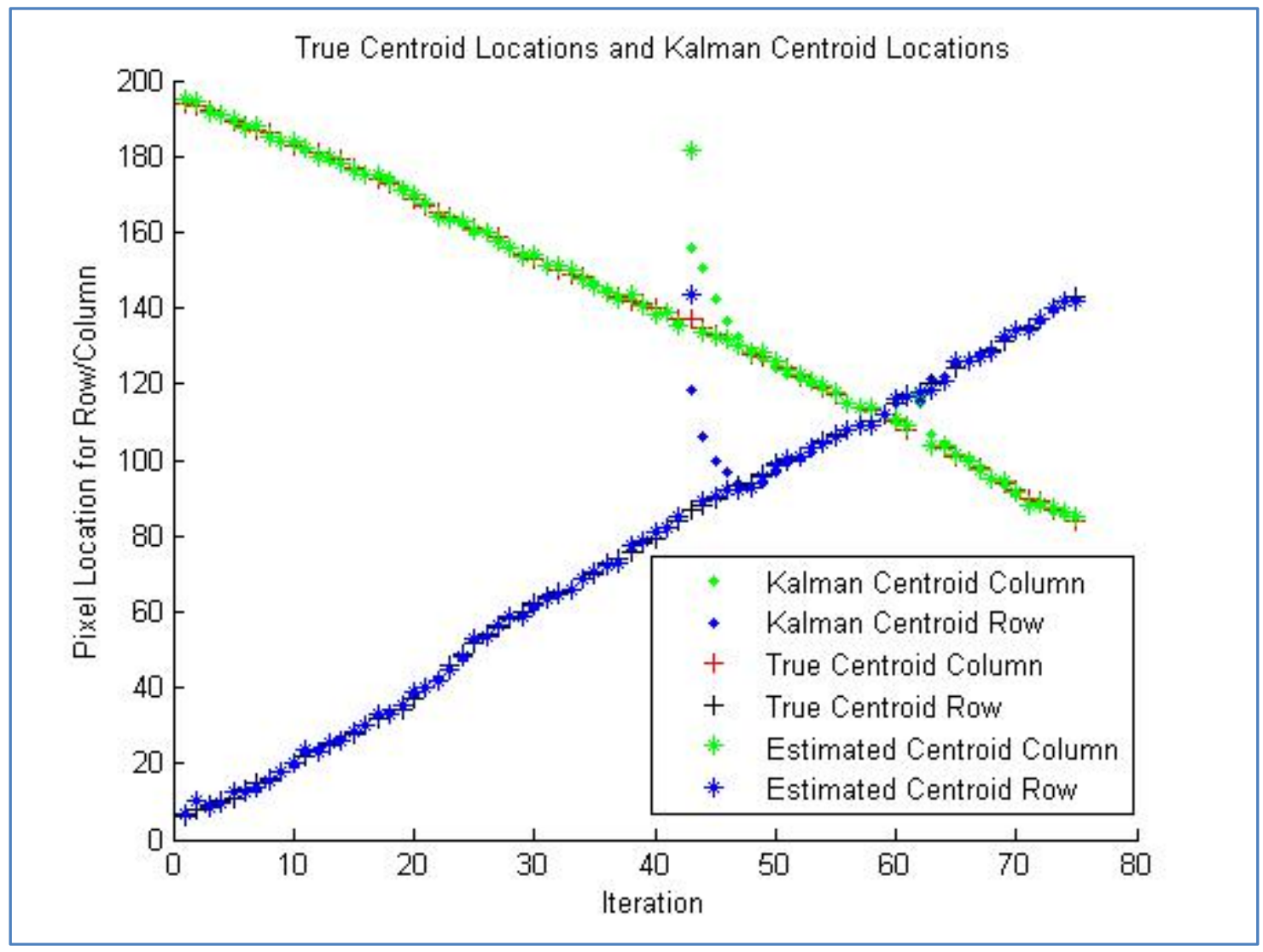

Figure 4.4: Awry Kalman Filter Example for Circular Target

In the case shown in Figure 4.4 above, the re-convergence to the path takes several iterations. Through experimentation, it was shown that this re-convergence time can be shortened or lengthened based on one's choice of the $\mathrm{H}$ matrix in the filter parameters. The $\mathrm{H}$ matrix is called the measurement matrix and relates the Kalman state to the current measurement. There are no noise effects included in the $\mathrm{H}$ matrix ${ }^{[2]}$. Larger values chosen to comprise the $\mathrm{H}$ matrix makes the system more dependent on dynamics rather than on the overall path. This translates to a longer re-convergence time. 


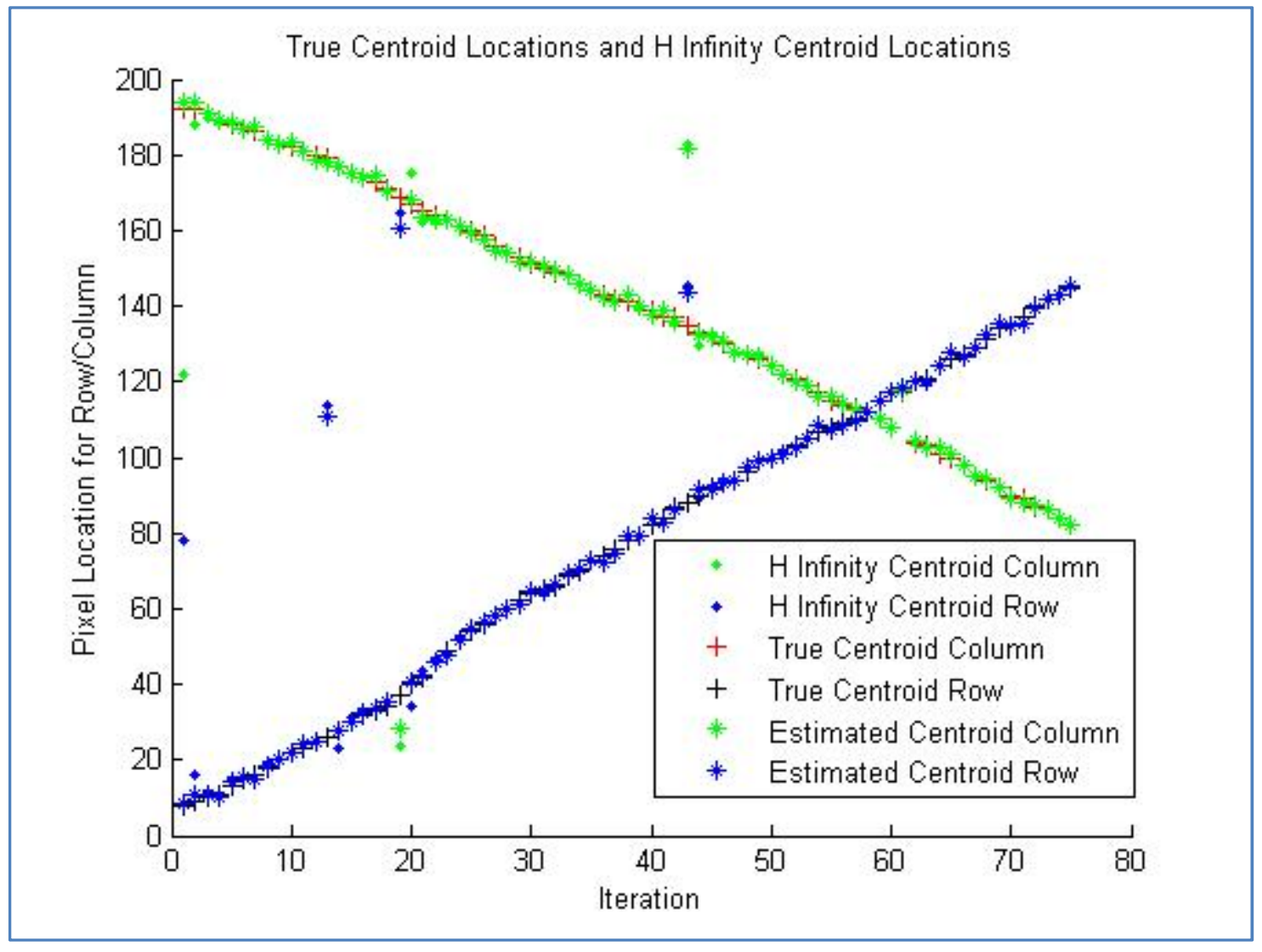

Figure 4.5: Awry H Infinity Filter Example for Circular Target

In Figure 4.5 above, it is easy to see the differences between the Kalman Filter results and the $\mathrm{H}$ Infinity Filter results. One can note that when a centroid is incorrectly located, the $\mathrm{H}$ Infinity filter appears to have more difficulty in returning to the target path compared to the Kalman Filter.

To experiment the effects of the above results, multiple test cases were run and the results are tabulated in Figures 4.6 through 4.11. The results in the tables are for averaged test results over 5 runs. 


\begin{tabular}{|c|c|c|c|c|c|}
\hline \multirow{2}{*}{\multicolumn{2}{|c|}{$\begin{array}{c}\text { Kalman Filter with } 5 \% \\
\text { Gaussian White Noise and } 1 \\
\text { pixel path error averaged } \\
\text { over } 5 \text { runs }\end{array}$}} & \multicolumn{2}{|c|}{ Circular Target } & \multicolumn{2}{|c|}{ Square Target } \\
\hline & & \multirow{2}{*}{$\begin{array}{c}\text { Mean } \\
\text { Squared } \\
\text { Error (pixels) }\end{array}$} & \multirow{2}{*}{$\begin{array}{c}\text { \# of } \\
\text { Incorrect } \\
\text { Centroid } \\
\text { Locations } \\
0\end{array}$} & \multirow{2}{*}{$\begin{array}{c}\text { Mean } \\
\text { Squared } \\
\text { Error (pixels) }\end{array}$} & \multirow{2}{*}{$\begin{array}{c}\text { \# of } \\
\text { Incorrect } \\
\text { Centroid } \\
\text { Locations } \\
0\end{array}$} \\
\hline \multirow{3}{*}{$\begin{array}{c}\text { Radius/Half } \\
\text { Edge Size }\end{array}$} & 6 pixels & & & & \\
\hline & 4 pixels & $\begin{array}{l}26.185-\text { row } \\
179.084-\text { col }\end{array}$ & 1.2 & $\begin{array}{c}0.5981-\text { row } \\
0.7136-\text { col }\end{array}$ & 0 \\
\hline & 2 pixels & $\begin{array}{c}5450.7 \text { - row } \\
3726.4 \text {-col }\end{array}$ & 68.6 & $\begin{array}{c}3076.7-\text { row } \\
3636.2 \text { - col }\end{array}$ & 58.0 \\
\hline
\end{tabular}

Figure 4.6: Simulated Point Targets for Kalman Filter with 5\% Noise

\begin{tabular}{|c|c|c|c|c|c|}
\hline \multirow{2}{*}{\multicolumn{2}{|c|}{$\begin{array}{c}\text { Kalman Filter with } 7.5 \% \\
\text { Gaussian White Noise and } 1 \\
\text { pixel path error averaged } \\
\text { over } 5 \text { runs }\end{array}$}} & \multicolumn{2}{|c|}{ Circular Target } & \multicolumn{2}{|c|}{ Square Target } \\
\hline & & \multirow{2}{*}{$\begin{array}{c}\text { Mean } \\
\text { Squared } \\
\text { Error (pixels) }\end{array}$} & \multirow{2}{*}{$\begin{array}{c}\text { \# of } \\
\text { Incorrect } \\
\text { Centroid } \\
\text { Locations } \\
0\end{array}$} & \multirow{2}{*}{$\begin{array}{c}\text { Mean } \\
\text { Squared } \\
\text { Error (pixels) } \\
0.6291 \text { - row } \\
0.6595 \text { - col }\end{array}$} & \multirow{2}{*}{$\begin{array}{c}\text { \# of } \\
\text { Incorrect } \\
\text { Centroid } \\
\text { Locations } \\
0\end{array}$} \\
\hline \multirow{3}{*}{$\begin{array}{c}\text { Radius/Half } \\
\text { Edge Size }\end{array}$} & 6 pixels & & & & \\
\hline & 4 pixels & $\begin{array}{c}205.20-\text { row } \\
408.77-\mathrm{col}\end{array}$ & 6.6 & $\begin{array}{l}0.9094-\text { row } \\
0.8352-\text { col }\end{array}$ & 0 \\
\hline & 2 pixels & $\begin{array}{c}4590.1-\text { row } \\
4177.3 \text { - col }\end{array}$ & 71.6 & $\begin{array}{c}3886.2 \text { - row } \\
3819.8 \text { - col }\end{array}$ & 60.6 \\
\hline
\end{tabular}

Figure 4.7: Simulated Point Targets for Kalman Filter with 7.5\% Noise 


\begin{tabular}{|c|c|c|c|c|c|}
\hline \multirow{2}{*}{\multicolumn{2}{|c|}{$\begin{array}{c}\text { Kalman Filter with } 10 \% \\
\text { Gaussian White Noise and } 1 \\
\text { pixel path error averaged } \\
\text { over } 5 \text { runs }\end{array}$}} & \multicolumn{2}{|c|}{ Circular Target } & \multicolumn{2}{|c|}{ Square Target } \\
\hline & & \multirow{2}{*}{$\begin{array}{c}\text { Mean } \\
\text { Squared } \\
\text { Error (pixels) }\end{array}$} & \multirow{2}{*}{$\begin{array}{c}\text { \# of } \\
\text { Incorrect } \\
\text { Centroid } \\
\text { Locations } \\
0\end{array}$} & \multirow{2}{*}{$\begin{array}{c}\text { Mean } \\
\text { Squared } \\
\text { Error (pixels) }\end{array}$} & \multirow{2}{*}{$\begin{array}{c}\text { \# of } \\
\text { Incorrect } \\
\text { Centroid } \\
\text { Locations } \\
0\end{array}$} \\
\hline \multirow{3}{*}{$\begin{array}{c}\text { Radius/Half } \\
\text { Edge Size }\end{array}$} & 6 pixels & & & & \\
\hline & 4 pixels & $\begin{array}{c}2016.5 \text { - row } \\
1644.2 \text {-col }\end{array}$ & 22.6 & $\begin{array}{c}1.0759-\text { row } \\
1.1433-\text { col }\end{array}$ & 0 \\
\hline & 2 pixels & $\begin{array}{c}4438.5 \text { - row } \\
3798.2 \text { - col }\end{array}$ & 70.0 & $\begin{array}{c}3939.0-\text { row } \\
5004.2-\text { col }\end{array}$ & 65.8 \\
\hline
\end{tabular}

Figure 4.8: Simulated Point Targets for Kalman Filter with $10 \%$ Noise

\begin{tabular}{|c|c|c|c|c|c|}
\hline \multirow{2}{*}{\multicolumn{2}{|c|}{$\begin{array}{c}\text { H Infinity Filter with } 5 \% \\
\text { Gaussian White Noise and } 1 \\
\text { pixel path error averaged } \\
\text { over } 5 \text { runs }\end{array}$}} & \multicolumn{2}{|c|}{ Circular Target } & \multicolumn{2}{|c|}{ Square Target } \\
\hline & & \multirow{2}{*}{$\begin{array}{c}\text { Mean } \\
\text { Squared } \\
\text { Error (pixels) } \\
4.9942-\text { row } \\
3.9596-\text { col }\end{array}$} & \multirow{2}{*}{$\begin{array}{c}\text { \# of } \\
\text { Incorrect } \\
\text { Centroid } \\
\text { Locations } \\
0\end{array}$} & \multirow{2}{*}{$\begin{array}{c}\text { Mean } \\
\text { Squared } \\
\text { Error (pixels) } \\
4.8961 \text { - row } \\
4.0473-\text { col }\end{array}$} & \multirow{2}{*}{$\begin{array}{c}\text { \# of } \\
\text { Incorrect } \\
\text { Centroid } \\
\text { Locations } \\
0\end{array}$} \\
\hline \multirow{3}{*}{$\begin{array}{c}\text { Radius/Half } \\
\text { Edge Size }\end{array}$} & 6 pixels & & & & \\
\hline & 4 pixels & $\begin{array}{l}5.9035 \text { - row } \\
3.5327 \text { - col }\end{array}$ & 2.2 & $\begin{array}{c}5.3178-\text { row } \\
4.1626-\text { col }\end{array}$ & 0 \\
\hline & 2 pixels & $\begin{array}{c}6010.4-\text { row } \\
4856.0-\mathrm{col}\end{array}$ & 71.9 & $\begin{array}{c}5005.7-\text { row } \\
4904.6-\text { col }\end{array}$ & 61.8 \\
\hline
\end{tabular}

Figure 4.9: Simulated Point Targets for $\mathrm{H}$ Infinity Filter with $5 \%$ Noise 


\begin{tabular}{|c|c|c|c|c|c|}
\hline \multirow{2}{*}{\multicolumn{2}{|c|}{$\begin{array}{c}\text { H Infinity Filter with } 7.5 \% \\
\text { Gaussian White Noise and } 1 \\
\text { pixel path error averaged } \\
\text { over } 5 \text { runs }\end{array}$}} & \multicolumn{2}{|c|}{ Circular Target } & \multicolumn{2}{|c|}{ Square Target } \\
\hline & & \multirow{2}{*}{$\begin{array}{c}\text { Mean } \\
\text { Squared } \\
\text { Error (pixels) }\end{array}$} & \multirow{2}{*}{$\begin{array}{c}\text { \# of } \\
\text { Incorrect } \\
\text { Centroid } \\
\text { Locations } \\
0\end{array}$} & \multirow{2}{*}{$\begin{array}{c}\text { Mean } \\
\text { Squared } \\
\text { Error (pixels) }\end{array}$} & \multirow{2}{*}{$\begin{array}{c}\text { \# of } \\
\text { Incorrect } \\
\text { Centroid } \\
\text { Locations } \\
0\end{array}$} \\
\hline \multirow{3}{*}{$\begin{array}{c}\text { Radius/Half } \\
\text { Edge Size }\end{array}$} & 6 pixels & & & & \\
\hline & 4 pixels & $\begin{array}{c}725.76-\text { row } \\
372.95 \text { - col }\end{array}$ & 6.4 & $\begin{array}{c}5.8446 \text { - row } \\
4.8412 \text { - col }\end{array}$ & 0.2 \\
\hline & 2 pixels & $\begin{array}{c}5481.1-\text { row } \\
5248.1 \text { - col }\end{array}$ & 70.8 & $\begin{array}{c}5408.6 \text { - row } \\
5306.9 \text { - col }\end{array}$ & 64.4 \\
\hline
\end{tabular}

Figure 4.10: Simulated Point Targets for H Infinity Filter with 7.5\% Noise

\begin{tabular}{|c|c|c|c|c|c|}
\hline \multirow{2}{*}{\multicolumn{2}{|c|}{$\begin{array}{c}\text { H Infinity Filter with } 10 \% \\
\text { Gaussian White Noise and } 1 \\
\text { pixel path error averaged } \\
\text { over } 5 \text { runs }\end{array}$}} & \multicolumn{2}{|c|}{ Circular Target } & \multicolumn{2}{|c|}{ Square Target } \\
\hline & & \multirow{2}{*}{$\begin{array}{c}\text { Mean } \\
\text { Squared } \\
\text { Error (pixels) } \\
5.5930 \text { - row } \\
4.5734 \text { - col }\end{array}$} & \multirow{2}{*}{$\begin{array}{c}\text { \# of } \\
\text { Incorrect } \\
\text { Centroid } \\
\text { Locations } \\
0\end{array}$} & \multirow{2}{*}{$\begin{array}{c}\text { Mean } \\
\text { Squared } \\
\text { Error (pixels) } \\
4.9340 \text { - row } \\
4.1356-\text { col }\end{array}$} & \multirow{2}{*}{$\begin{array}{c}\text { \# of } \\
\text { Incorrect } \\
\text { Centroid } \\
\text { Locations } \\
0\end{array}$} \\
\hline \multirow{3}{*}{$\begin{array}{c}\text { Radius/Half } \\
\text { Edge Size }\end{array}$} & 6 pixels & & & & \\
\hline & 4 pixels & $\begin{array}{c}1632.6-\text { row } \\
1905.0-\mathrm{col}\end{array}$ & 22.2 & $\begin{array}{c}85.977 \text { - row } \\
9.8845 \text { - col }\end{array}$ & 0.4 \\
\hline & 2 pixels & $\begin{array}{c}6698.9-\text { row } \\
5056.7-\text { col }\end{array}$ & 72.2 & $\begin{array}{c}5343.5 \text { - row } \\
5081.4 \text { - col }\end{array}$ & 64.8 \\
\hline
\end{tabular}

Figure 4.11: Simulated Point Targets for H Infinity Filter with $10 \%$ Noise 
As can be seen in Figures 4.6 through 4.11, the results for both the circular and square target were consistent for both the Kalman and $\mathrm{H}$ Infinity filters. As noise increased, so did the number of incorrect centroid locations. It can be seen that the largest discontinuity in the incorrect prediction of centroid locations was between $7.5 \%$ Gaussian White Noise and 10\% Gaussian White Noise, whereas the difference in results between $5 \%$ and $7.5 \%$ was much less harsh.

As expected, the square target outperformed the circular target. This is because for any given radius/half edge size, the square will actually comprise of more white pixels than the circle will, making the algorithm used to find the target more accurate.

Neither filter severely outperformed the other and thus proves that both the Kalman and $\mathrm{H}$ Infinity filters are viable tracking filters for this type of exaggerated point target. 


\section{Prediction and Matching Detection Filter Point Target}

\section{Tracking}

\subsection{Introduction}

Chapter 4 discussed circular and square targets as they became progressively smaller, with the smallest target being a 4 pixel diameter circle and a 4 pixel wide square. The results show that neither the Kalman filter nor the $\mathrm{H}$ Infinity filter accurately performs for a target that small using the experimental methods provided in this thesis. This chapter will examine algorithms that can be used on the smallest of all point targets-a single pixel.

Initial work on detection of a single pixel moving point target is called the Track-BeforeDetect (TBD) algorithm ${ }^{[7]}$. The method is still being improved since it is currently impractical because of the amount of time used for computation to search the entire image space for all possible trajectories for each target and their corresponding velocities. Currently there is work being performed to experiment with new techniques for target detection such as: using the wavelet function, experimenting with neural networks, and attempting multiple feature fusion ${ }^{[8]}$.

This thesis explores a type of algorithm called the Prediction and Matching Detection Algorithm (PAMD). It relies on a very high image frame rate ${ }^{[18]}$ and assumes that the point target is moving in a fairly straight path given a short amount of time. The filter first makes a prediction about the target's location based on the target's history. Then 
the algorithm looks for a target at or near the predicted location to determine its path. This type of algorithm classifies the PAMD as a track before detect (TBD) algorithm.

\subsection{Prediction and Matching Detection Algorithm}

\subsubsection{PAMD Test Image Creation}

To perform the PAMD filter testing, 26 sample images were created. Each image was $128 \times 128$ pixels and was comprised of 10\% Gaussian White Noise. This Gaussian White Noise is to simulate the process and speckle noise present in practical SAR image generation scenarios. Speckle noise is caused by constructive interference from the processing of multiple wave front data points in the raw SAR data. An object in the images target region produces radar backscatter due to multiple pulses and is postprocessed multiple times-sometimes it is destructive interference and the pixel value is too low, and other times it is constructive interference and the pixel value is too high. Each image contained a single point target pixel with an intensity brighter than all other pixels in the image. Successive images contained the same point target at a distance less than 3 pixels away in all dimensions to simulate a high frame-rate sensing system. During one of the frames, the pixel is moved to a location completely out of the expected path to test the filter for resilience to extremely noisy input. A sample image is shown in Figure 5.1. 


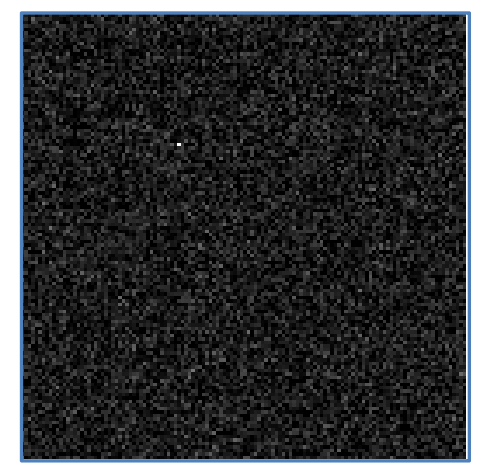

Figure 5.1: Sample Test Image for the PAMD Filter

\subsubsection{PAMD Algorithm Description}

The start of the point target's track is found by locating the pixel with the maximum grey level in the first frame. This pixel is then tracked in all subsequent frames. The following matrices are created:

- Position (26x2): Holds the current $(x, y)$ position of the tracked path

- Predicted Position (26x2): Holds the projected position for the next time frame.

- Match Flag (26x1): Set to a ' 1 ' if a point target was found in the image frame for a given time.

- Match Number (26x1): Holds the consecutive number of matched predictions to the actual track point

- Consecutive Predicted Number (26x1): Holds the consecutive number of failures to determine when/if to drop out of the algorithm.

The algorithm works by using the changes from the previous two frames to predict the most likely position in the next frame. This position is then used as the center of a $7 x 7$ 
mask region to search for a point target in the next frame. The equations used to compute the predicted position of the point target in the next frame (the center of the $7 \times 7$ mask) are:

$$
\begin{aligned}
& \text { Predicted Position }_{k+1}(x) \\
& =\text { Current Position }_{k}(x)+d x_{1}+\left(d x_{1}-d x_{2}\right) \\
& \text { Predicted Position }_{k+1}(x) \\
& =\text { Current Position }_{k}(x)+d x_{1}+\left(d x_{1}-d x_{2}\right)
\end{aligned}
$$

In these formulas, the index $k$ represents the number of the image frame currently being read in. The differential terms $d x_{1}$ and $d y_{1}$ represent the change in position between the current frame and the previous frame, whereas $d x_{2}$ and $d y_{2}$ represent the change in position between frames $k-1$ and $k-2$. The $\left(d x_{1}-d x_{2}\right)$ and $\left(d y_{1}-d y_{2}\right)$ terms are not included in the formulas for $k<3$.

Figure 5.2 shows this set of computations in graphical form.

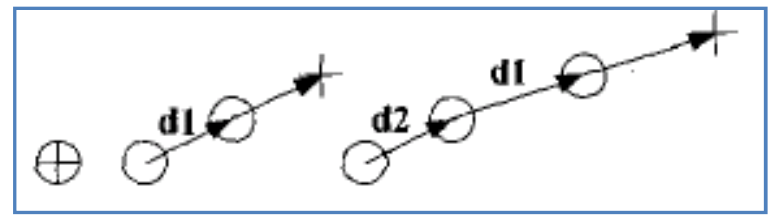

Figure 5: Predicting Process (Circles represent known position, crosses represent predicted position) 
For each $k$ frame, once the position of the target track for frame $k+1$ is predicted, the $k+1$ frame is read in and sent to a findPixel () algorithm. This algorithm searches for the point target in a confined region surrounding the predicted position. The region is confined based on two parameters: distance from the predicted position and the current angle of the track path. The searched region is initially confined to a $7 \times 7$ pixel mask around the predicted position, but any point targets found in this mask region must also be located with $45^{\circ}$ (on either side) of the angle between the previous two track positions. This ensures that the maximum change in track path is a reasonable figure of $45^{\circ}$. In a high frame-rate system, the angle of the track path would not be changing significantly between individual frames.

If no point target is found in the allowable subsection of the image, then it is assumed the current $k+1$ frame represents a very noisy measurement, and no point target location is extracted. Instead, the location of the predicted position is used as the current position for the $k+1$ time index.

After a specifiable number of consecutive "failures" (in which no point target was found and the predicted position was used as the next current position), the algorithm's tracked path is no longer reliable since it is still using the dynamics $\left(d x_{1}, d y_{1}, d x_{2}, d y_{2}\right)$ from several frames prior and no new input has been obtained. At this point, the algorithm should quit to avoid reporting unreliable information. A flowchart showing the steps taken in the algorithm can be found in Figure 5.3. 


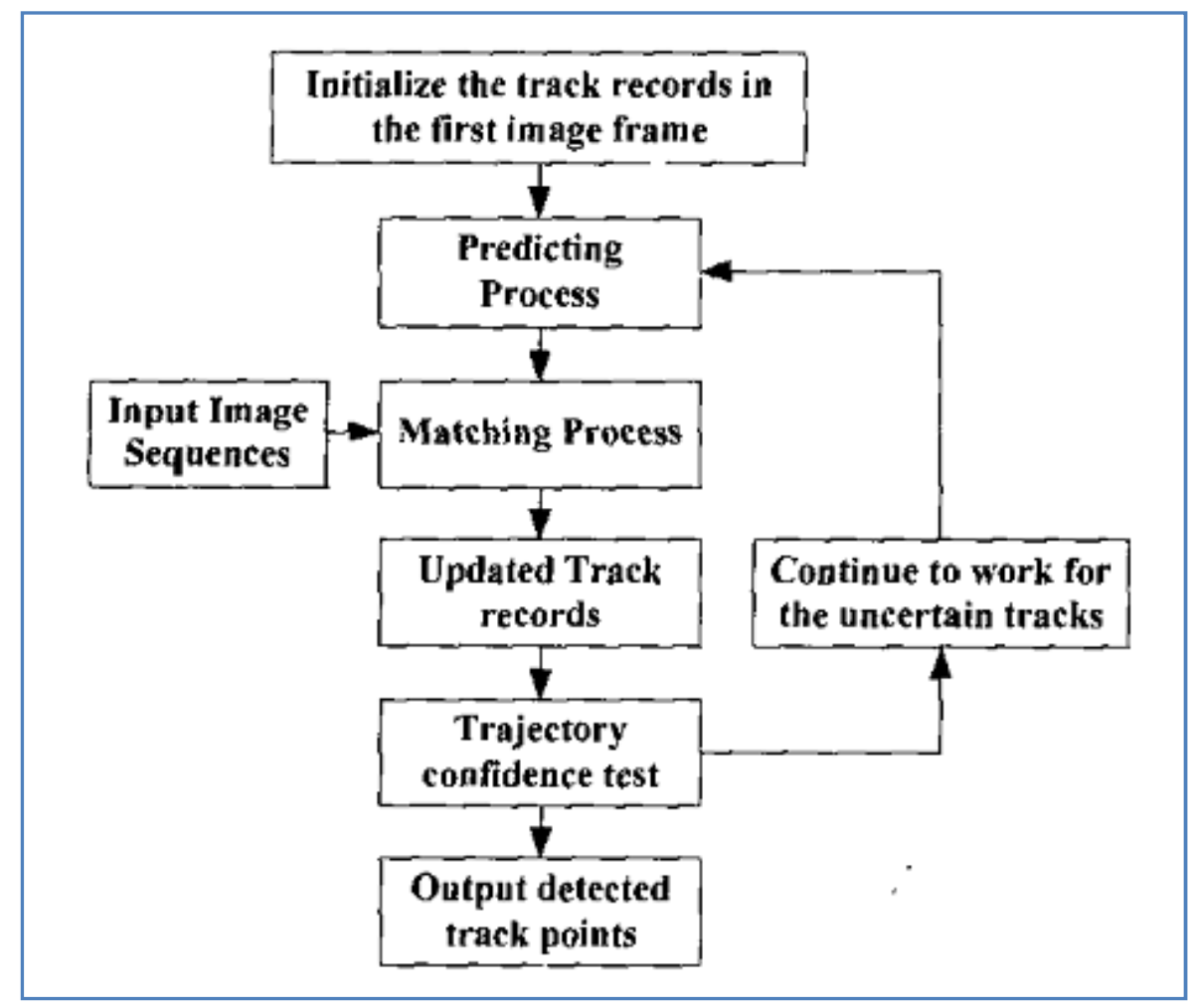

Figure 5.3: Flowchart of the PAMD Filter Algorithm

\subsection{PAMD Filter Simulation Results for Single Point Target Tracking}

The PAMD filter results are based on the set of 26 input images described in Figure 5.4. Gaussian white noise of $10 \%$ of the pixels is added to each frame. There is also an instance when the target temporarily disappears for one frame-indicated by bold font in Figure 5.4. Figure 5.4 illustrates the actual point target location and the filter's estimated point target location. Figure 5.5 displays the path of the point target. 


\begin{tabular}{|c|c|c|}
\hline Frame Number & $\begin{array}{l}\text { Actual Point Target } \\
\text { Location }\end{array}$ & $\begin{array}{l}\text { Computed PAMD Point } \\
\text { Target Location }\end{array}$ \\
\hline 1 & $(20,12)$ & $(20,12)$ \\
\hline 2 & $(22,14)$ & $(22,14)$ \\
\hline 3 & $(24,16)$ & $(24,16)$ \\
\hline 4 & $(26,18)$ & $(26,18)$ \\
\hline 5 & $(28,20)$ & $(28,20)$ \\
\hline 6 & $(30,23)$ & $(30,23)$ \\
\hline 7 & $(32,25)$ & $(32,25)$ \\
\hline 8 & $(34,26)$ & $(34,26)$ \\
\hline 9 & $(36,28)$ & $(36,28)$ \\
\hline 10 & $(38,30)$ & $(38,30)$ \\
\hline 11 & $(1,1)$ & $(40,32)$ \\
\hline 12 & $(42,34)$ & $(42,34)$ \\
\hline 13 & $(44,36)$ & $(44,36)$ \\
\hline 14 & $(46,38)$ & $(46,38)$ \\
\hline 15 & $(48,40)$ & $(48,40)$ \\
\hline 16 & $(50,42)$ & $(50,42)$ \\
\hline 17 & $(52,44)$ & $(52,44)$ \\
\hline 18 & $(54,46)$ & $(54,46)$ \\
\hline 19 & $(56,48)$ & $(56,48)$ \\
\hline 20 & $(59,51)$ & $(59,51)$ \\
\hline 21 & $(60,52)$ & $(60,52)$ \\
\hline 22 & $(62,54)$ & $(62,54)$ \\
\hline 23 & $(64,56)$ & $(64,56)$ \\
\hline 24 & $(66,58)$ & $(66,58)$ \\
\hline 25 & $(68,60)$ & $(68,60)$ \\
\hline 26 & $(70,63)$ & $(70,63)$ \\
\hline
\end{tabular}

Figure 5.4: PAMD Filter Results 


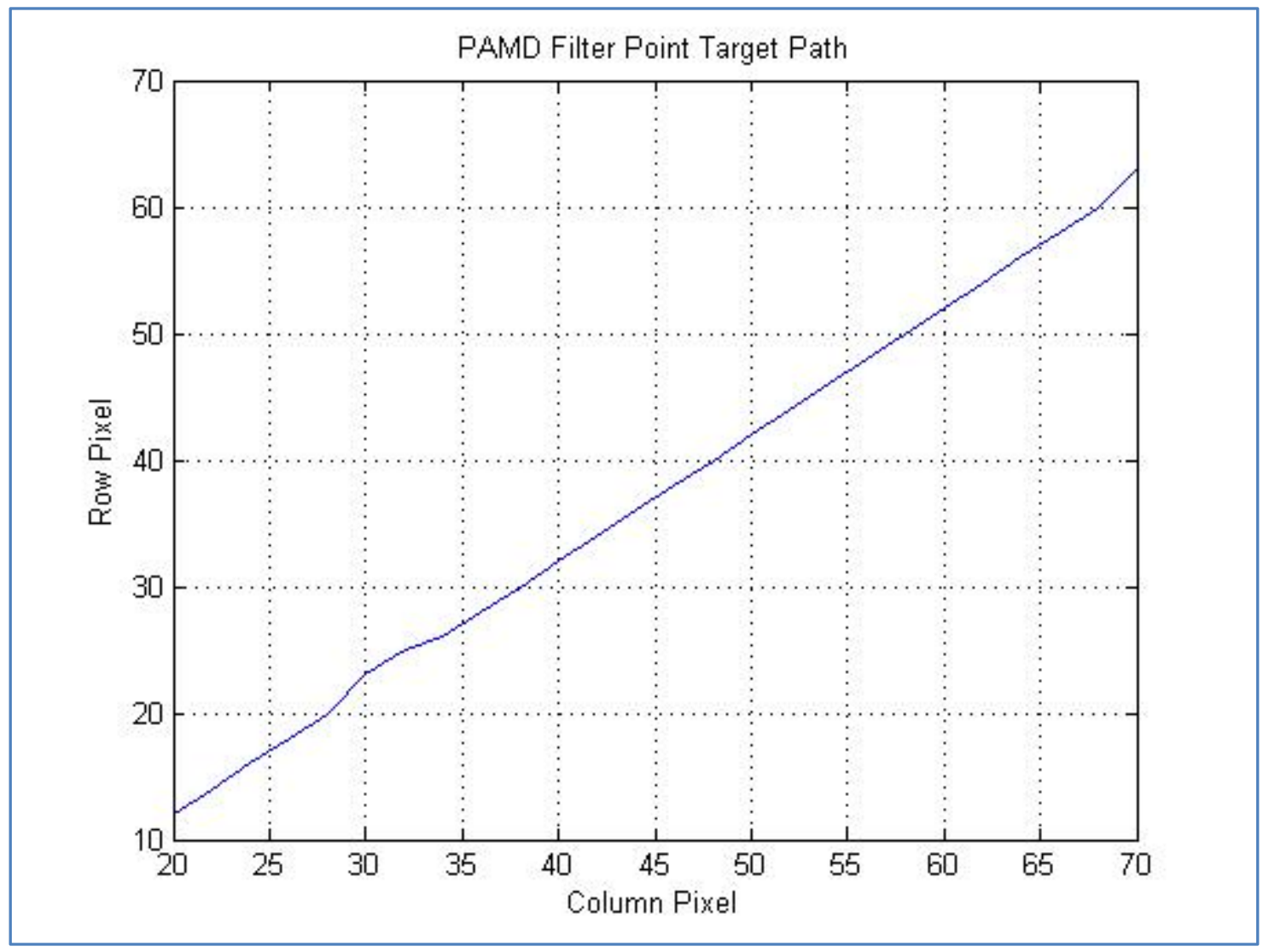

Figure 5.5: PAMD Filter Single Point Target Path

\subsection{PAMD Filter Simulation Results for Multiple Point Target Tracking}

The PAMD filter algorithm can be extended for use on multiple point targets as well, since the required information used to track is based on the projected path angle. Two targets traveling with different path angles are fairly easy to distinguish and track using the PAMD filter algorithm.

Similarly to the single point target case, 26 input images are created, each with two point targets in them. As with the single point target, $10 \%$ Gaussian noise is added to the image and there are also several instances of a "missing point target", where the 
target temporarily disappears for a frame-indicated by bold font in Figure 5.6. Figure

5.6 illustrates the actual point target locations and the computed point target locations

(using the PAMD filter algorithm). Figure 5.7 displays the path of the point targets.

\begin{tabular}{|c|c|c|c|c|}
\hline Frame Number & $\begin{array}{l}\text { Actual Point } \\
\text { Target } 1 \\
\text { Location }\end{array}$ & $\begin{array}{c}\text { Computed } \\
\text { PAMD Point } \\
\text { Target } 1 \\
\text { Location }\end{array}$ & $\begin{array}{l}\text { Actual Point } \\
\text { Target } 2 \\
\text { Location }\end{array}$ & $\begin{array}{c}\text { Computed } \\
\text { PAMD Point } \\
\text { Target } 2 \\
\text { Location }\end{array}$ \\
\hline 1 & $(25,103)$ & $(25,103)$ & $(28,20)$ & $(28,20)$ \\
\hline 2 & $(27,101)$ & $(27,101)$ & $(30,22)$ & $(30,22)$ \\
\hline 3 & $(1,1)$ & $(29,99)$ & $(32,24)$ & $(32,24)$ \\
\hline 4 & $(31,97)$ & $(31,97)$ & $(34,26)$ & $(34,26)$ \\
\hline 5 & $(33,95)$ & $(33,95)$ & $(36,28)$ & $(36,28)$ \\
\hline 6 & $(35,93)$ & $(35,93)$ & $(38,30)$ & $(38,30)$ \\
\hline 7 & $(37,91)$ & $(37,91)$ & $(1,1)$ & $(40,32)$ \\
\hline 8 & $(39,89)$ & $(39,89)$ & $(42,34)$ & $(42,34)$ \\
\hline 9 & $(41,87)$ & $(41,87)$ & $(44,36)$ & $(44,36)$ \\
\hline 10 & $(43,85)$ & $(43,85)$ & $(46,38)$ & $(46,38)$ \\
\hline 11 & $(45,83)$ & $(45,83)$ & $(48,40)$ & $(48,40)$ \\
\hline 12 & $(47,81)$ & $(47,81)$ & $(50,42)$ & $(50,42)$ \\
\hline 13 & $(49,79)$ & $(49,79)$ & $(52,44)$ & $(52,44)$ \\
\hline 14 & $(121,121)$ & $(51,77)$ & $(54,46)$ & $(54,46)$ \\
\hline 15 & $(53,75)$ & $(53,75)$ & $(56,48)$ & $(56,48)$ \\
\hline 16 & $(55,73)$ & $(55,73)$ & $(58,50)$ & $(58,50)$ \\
\hline 17 & $(57,71)$ & $(57,71)$ & $(60,52)$ & $(60,52)$ \\
\hline 18 & $(59,69)$ & $(59,69)$ & $(62,54)$ & $(62,54)$ \\
\hline 19 & $(61,67)$ & $(61,67)$ & $(64,56)$ & $(64,56)$ \\
\hline 20 & $(63,65)$ & $(63,65)$ & $(66,58)$ & $(66,58)$ \\
\hline 21 & $(65,63)$ & $(65,63)$ & $(68,60)$ & $(68,60)$ \\
\hline 22 & $(67,61)$ & $(67,61)$ & $(70,62)$ & $(70,62)$ \\
\hline 23 & $(69,59)$ & $(69,59)$ & $(72,64)$ & $(72,64)$ \\
\hline 24 & $(71,57)$ & $(71,57)$ & $(74,66)$ & $(74,66)$ \\
\hline 25 & $(73,55)$ & $(73,55)$ & $(76,68)$ & $(76,68)$ \\
\hline 26 & $(74,53)$ & $(75,53)$ & $(78,70)$ & $(78,70)$ \\
\hline
\end{tabular}

Figure 5.6: PAMD Filter Results with Two Targets 


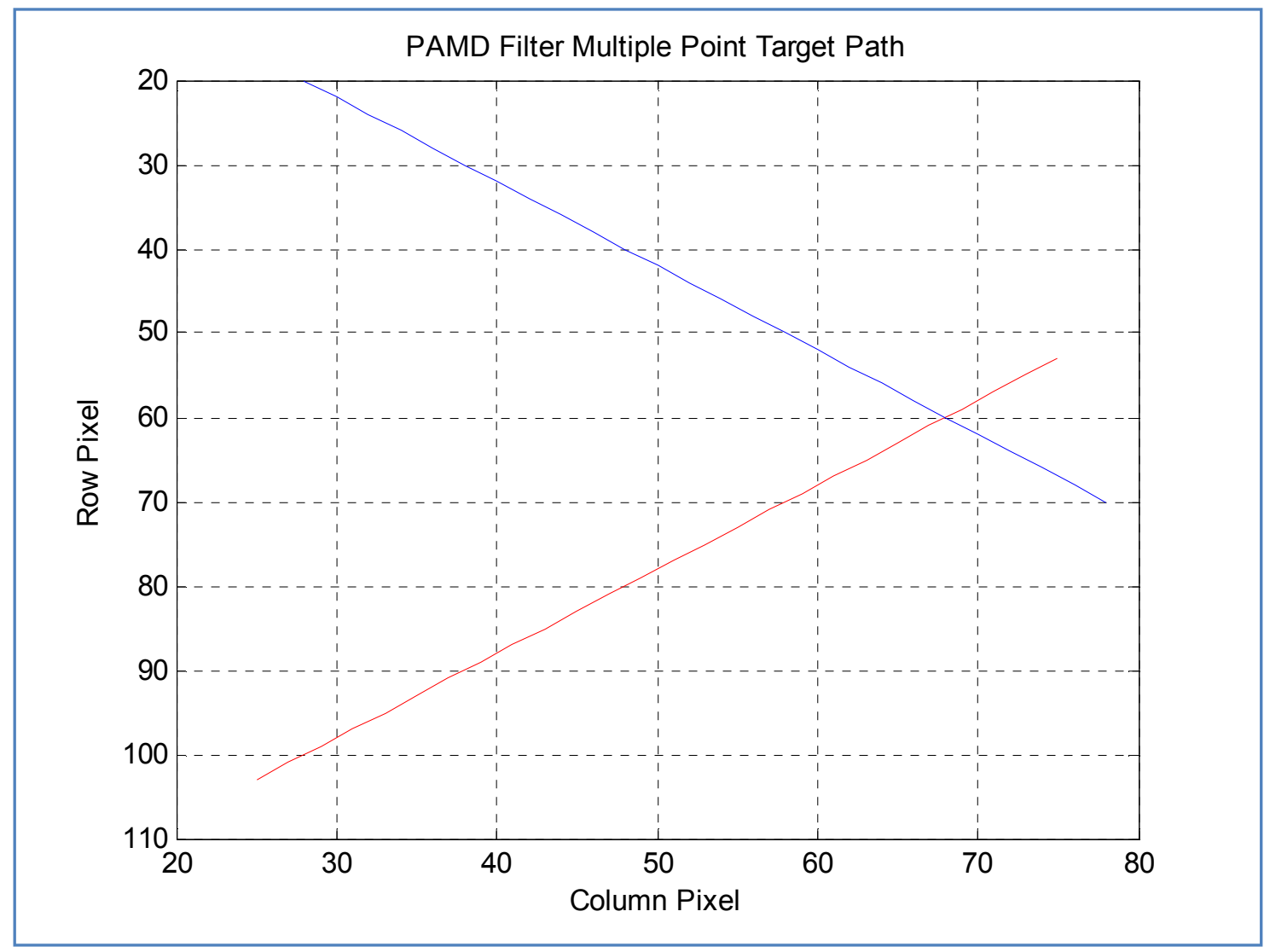

Figure 5.7: PAMD Filter Multiple Point Target Path

\subsection{Limitations of the PAMD Filter Algorithm}

While the PAMD Filter algorithm proves to be a useful filter, there are several limitations which may be important when dealing with SAR systems. Most importantly, the paths need to be very constant, which also relies on a very high frame rate. True SAR images take several seconds to process and the time delay may be too long to work accurately with a SAR platform since the frame rate may be too slow to be compatible with the PAMD Filter. 
Another limitation is the sensitivity of the filter. If paths are not almost entirely linear, the filter will go awry and become unstable yielding unwanted results. This is not a problem for very high frame rate systems, however as mentioned above, this may not always be possible with a given SAR platform.

When tracking multiple targets, the PAMD Filter does an adequate job distinguishing between the two targets for the very general case used in this thesis. However, there are many circumstances which might cause the filter to go awry when tracking multiple targets. The two targets may get mixed up when they cross if the path angle of both targets is similar, since that is the only real difference between the two targets. Another target may also enter the trajectory of one of the initial targets and the PAMD Filter algorithm by itself does not provide any sort of target recognition to determine if the targets being tracked are the same ones over time.

In conclusion, the PAMD Filter algorithm is viable method for high frame-rate systems with fairly constant velocities. The PAMD Filter algorithm has the ability to track multiple targets as well, but would function much better with some sort of target recognition system in addition to the filter itself. 


\section{Raw SAR Target Tracking in the Slow-Time Domain}

\subsection{Moving Target Imaging Without Compensation}

In 2007, Cal Poly Master's Student Brian Zaharris completed his thesis on the Range Doppler Algorithm and successfully demonstrated the deficiencies in the algorithm when dealing with a moving target. His analysis was limited to targets moving in the positive range direction or positive azimuth direction. To show that some sort of compensation would be needed when dealing with moving targets as opposed to stationary targets, Brian performed the same range Doppler techniques on moving data as he did on stationary data ${ }^{[17]}$.

His results showed that by using the Range Doppler Algorithm intended for stationary targets it was impossible to resolve both the range and azimuth directions to accurately image the final target. Instead, a blur of data covering an area of approximately $20 \times 60$ meters was imaged. The final imaged target without compensation can be seen in Figure 6.1. 


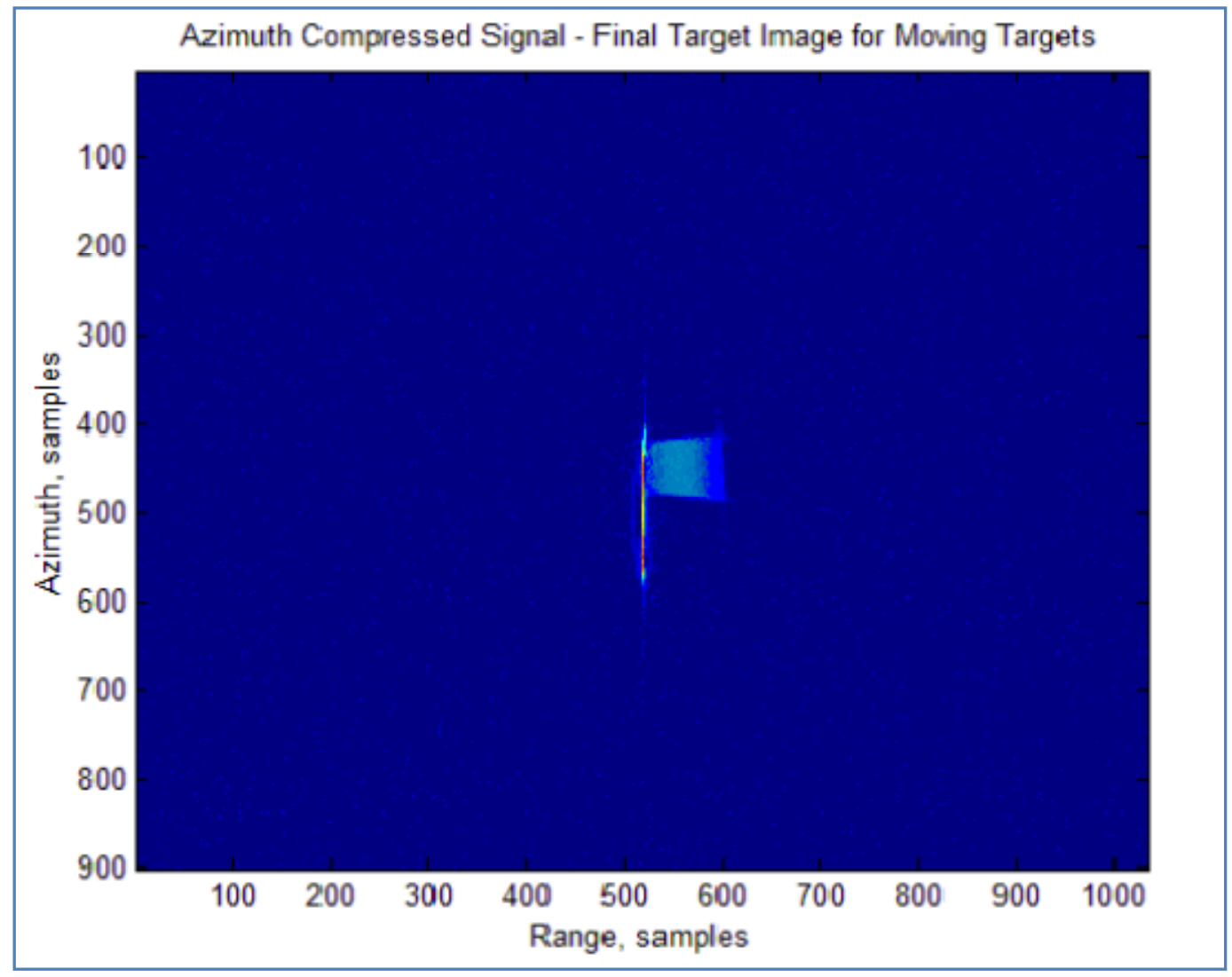

Figure 6.1: Final target image for two moving targets without compensation

To compensate for the incorrect imaging, Brian used the dynamics of the moving system to derive two equations for the amount of movement in both the range and azimuth directions based on the slant range. These metrics were used as the input to a 1-D Kalman filter which tracked the first three seconds of a SAR moving target and then validated the tracking method by performing a final image of the targets to show that they could be resolved and imaged using his correction method.

The correction method used in Brian's thesis was two equations that accounted for additional movement in the range and azimuth directions. Once an initial movement was detected, all subsequent movement was then calculated and input into the 1-D 
Kalman filter. The following equations were used as movement measurements in the range direction and azimuth direction based on the slant range, respectively.

$$
\begin{aligned}
& y(n)= \pm \sqrt{R(n)^{2}-R_{o}^{2}}-V p\left[\frac{d u r}{2}-\eta(n)\right] \\
& x(n)=\sqrt{R(n)^{2}-V p^{2}\left[\frac{d u r}{2}-\eta(n)\right]^{2}}-R_{o}
\end{aligned}
$$

Where $\mathrm{x}$ and $\mathrm{y}$ are measured in meters and are a function of the sample number, $n . \mathrm{R}(n)$ is the measured instantaneous slant range on the ground plane. In the equation for the range movement, the plus sign changed to a minus sign after the aircraft passed the range of closest approach. The goal of this slant range correction is to make it appear as if the target has been stationary in its final position over all time ${ }^{[17]}$.

\subsection{Modifications to the Slant Range Correction Process}

The goal in this thesis is to accurately track the slow time target in two dimensions instead of one. This allows for a more realistic situation since it is unlikely that a target's instantaneous velocity will be in only one direction. To add the ability for a second dimension to be tracked, a second parameter measurement is required.

Using only the slant range as an input parameter to obtain instantaneous movement measurements presents a problem when movement is expanded to two dimensions. In this situation, the geometric relationship used to derive measurement equations is unsolvable, as there are two unknowns for only one input equation. Various 
approximations of some parameters could be used to make rough estimations of the measurements, but these estimations would be extremely inaccurate and not useful in a tracking or filtering application. This inaccuracy is due to the fact that the measured differences between instantaneous and expected slant ranges (and other parameters) are on the order of less than a meter out of 20 kilometers between individual samples.

One example of a rough estimation is to assume during the individual measurements of range and azimuth movement that there was no movement in the other direction. The formulas for computing the measurements then default to the same equations that Brian Zaharris used, but the value of the input parameter will be different due to the change in the target position in both dimensions, and the actual filtering operation (Kalman, $\mathrm{H}$ Infinity or other) will have to account for both dimensions. Figures 6.2 and 6.3 show the result of making this assumption, in this case with positive movement in both range and azimuth dimensions. 


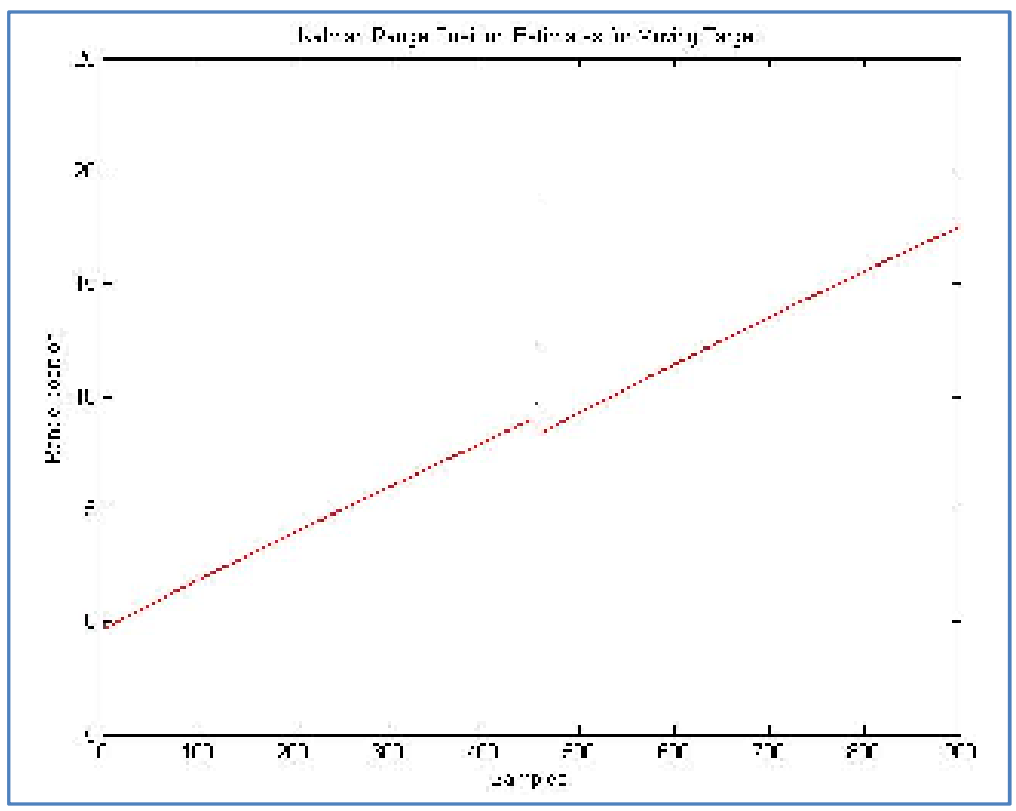

Figure 6.2: Range tracked position estimates with 2-D motion and only a single input parameter

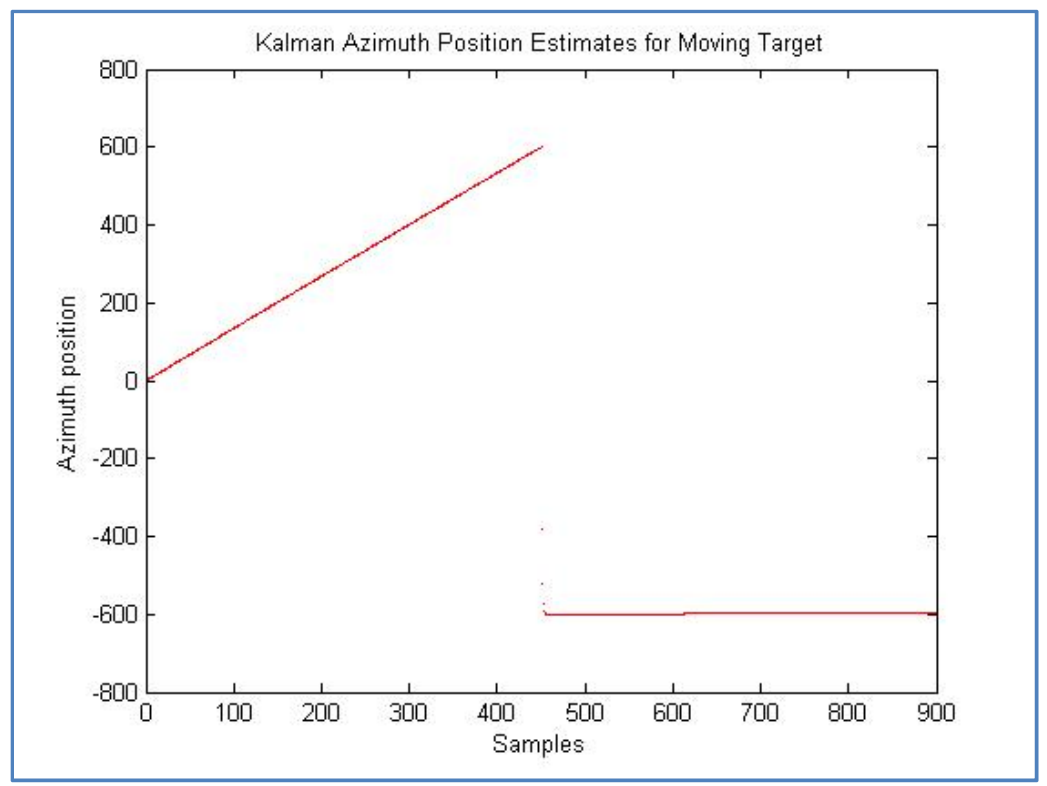

Figure 6.3: Azimuth tracked position estimates with 2-D motion and only a single input parameter

As can be seen in these two figures, the 2-D tracking filter (Kalman in this case) was able to adequately track movement in the range dimension. The measurements (and 
therefore tracking estimates) in the azimuth dimension, however, are extremely poorly scaled, and the switch between positive and negative for the square root did nothing to account for the platform's position change relative to the target. For this reason, making rough estimates or assumptions about input parameters yields far too large of an error in the measurement inputs to the tracking filters.

The most obvious solution for this problem is to introduce a second available radar parameter as an input to the measurement equations. Using the squint angle in conjunction with the slant range, it is simple geometry to derive new equations using both of these variables to allow one to solve for the target position in both the range and azimuth dimensions. The equations presented in section 6.1 will yield the same (or similar) measurement formulas as these new equations when motion is only determined to be in one dimension.

The basic geometric relationships used to derive measurement formulas based on the instantaneous slant range and squint angle are:

$$
\begin{gathered}
R(n)^{2}=\left[R_{o}+x(n)\right]^{2}+\left[V p\left(\frac{d u r}{2}\right)-V p\left(\frac{n}{P R F}\right)+y(n)\right]^{2} \\
\theta_{s q}=\tan ^{-1}\left[\frac{\left|V p\left(\frac{d u r}{2}\right)-V p\left(\frac{n}{P R F}\right)+y(n)\right|}{R_{o}+x(n)}\right]
\end{gathered}
$$


From these relationships, solutions can be found for $x(n)$ and $y(n)$ in terms of the constant platform parameters and the instantaneous measured parameters $R(n)$ and $\theta_{s q}$. These final equations are defined as:

$$
\begin{aligned}
& y(n)= \pm \sqrt{\frac{R(n)^{2} \tan ^{2}\left(\theta_{s q}(n)\right)}{1+\tan ^{2}\left(\theta_{s q}(n)\right)}}+V p\left(\frac{n}{P R F}\right)-V p\left(\frac{d u r}{2}\right) \\
& x(n)=\sqrt{R(n)^{2}-V p^{2}\left[\frac{d u r}{2}-V p\left(\frac{n}{P R F}\right)+y(n)\right]^{2}}-R_{o}
\end{aligned}
$$

Figure 45 describes what each of the variables and parameters in the above equations are $^{[5][13]}$. These are the set of parameters that are known by nature of the platform and imaging situation, or are measured at sample time. All of them are required to compute $x(n)$ and $y(n)$, the measurement inputs to the tracking filter for each sample.

\begin{tabular}{|c|c|}
\hline$R(n)$ & The instantaneous slant range at sample index $n$, in meters. \\
\hline$\theta_{s q}(n)$ & The instantaneous squint angle at sample index $n$. \\
\hline$V p$ & Velocity of the platform in meters per second. \\
\hline$P R F$ & Pulse Repetition Frequency of the radar signal in Hertz. \\
\hline$d u r$ & Duration time of the SAR imaging scenario, in seconds. \\
\hline$n$ & Sampling index in slow-time domain. \\
\hline
\end{tabular}

Figure 6.4: Variable and parameter descriptions for measurement formulas 


\subsection{SAR Target Tracking Using Squint Angle and Slant Range}

Brian Zaharris's MATLAB code ${ }^{[17]}$ was updated to include the new measurement equations as well as a two-dimensional Kalman filter. The user has the ability to set a velocity in the range direction as well as a velocity in the azimuth direction. Without any additional noise added in, the Kalman filter functions as desired given the inputs $x(n)$ and $y(n)$. Using the original Range Doppler Algorithm, the final target image is obtainable, in contrast to the target in Figure 6.1 which was not obtainable due to the lack of squint angle inclusion.

With a constant range velocity of $12 \mathrm{~m} / \mathrm{s}$ and a constant azimuth velocity of $3 \mathrm{~m} / \mathrm{s}$, the resulting images in Figures 6.5 through 6.9 show that the updated MATLAB code functions as expected. Figures 6.5 and 6.6 show the Kalman estimated position over the first 3 seconds or 900 samples. Figures 6.7 and 6.8 show the Kalman estimated velocity over the same time period of 900 samples. Figure 6.9 shows the final target imaged. 


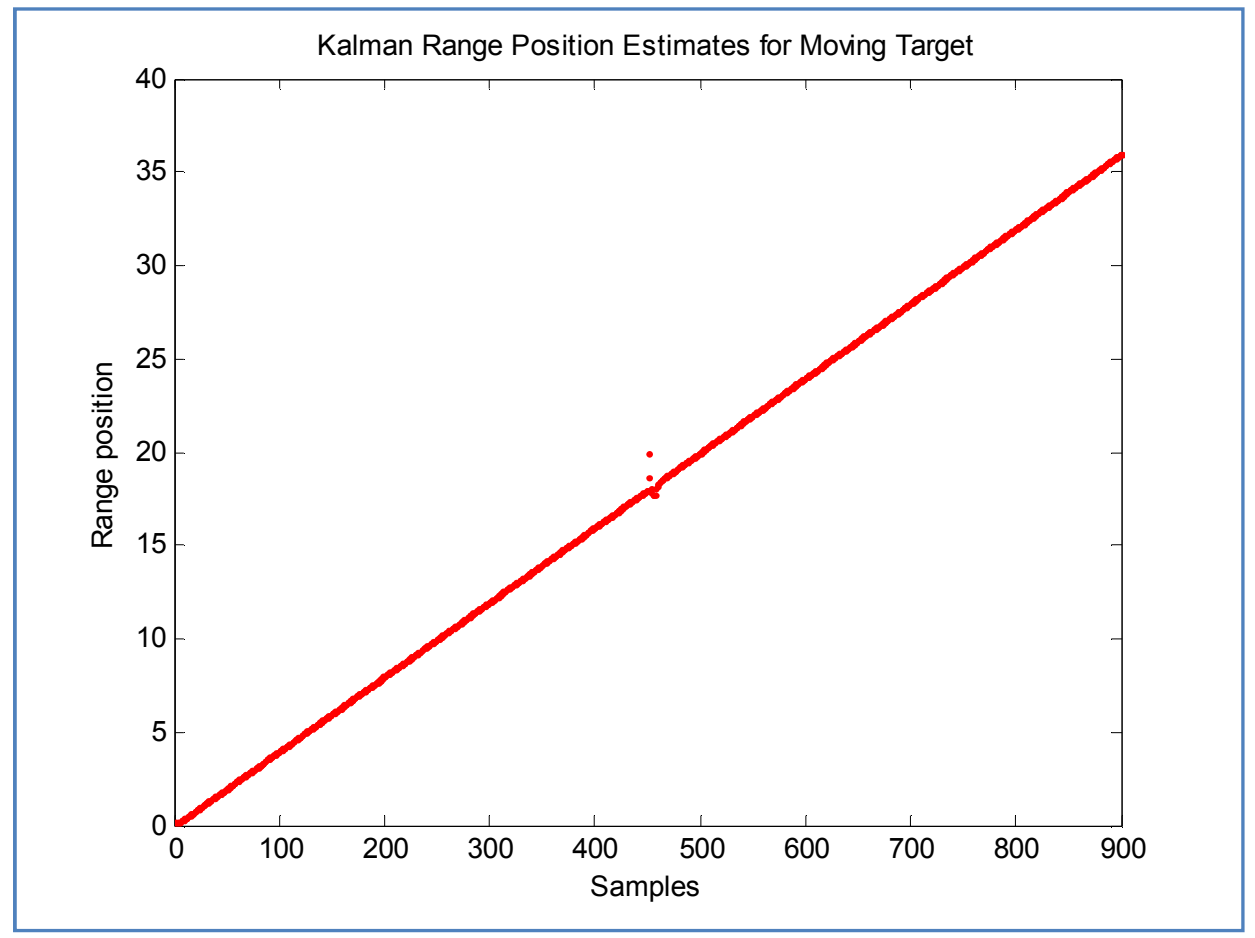

Figure 6.5: Kalman Range Position Estimates for 2-D Moving Target 


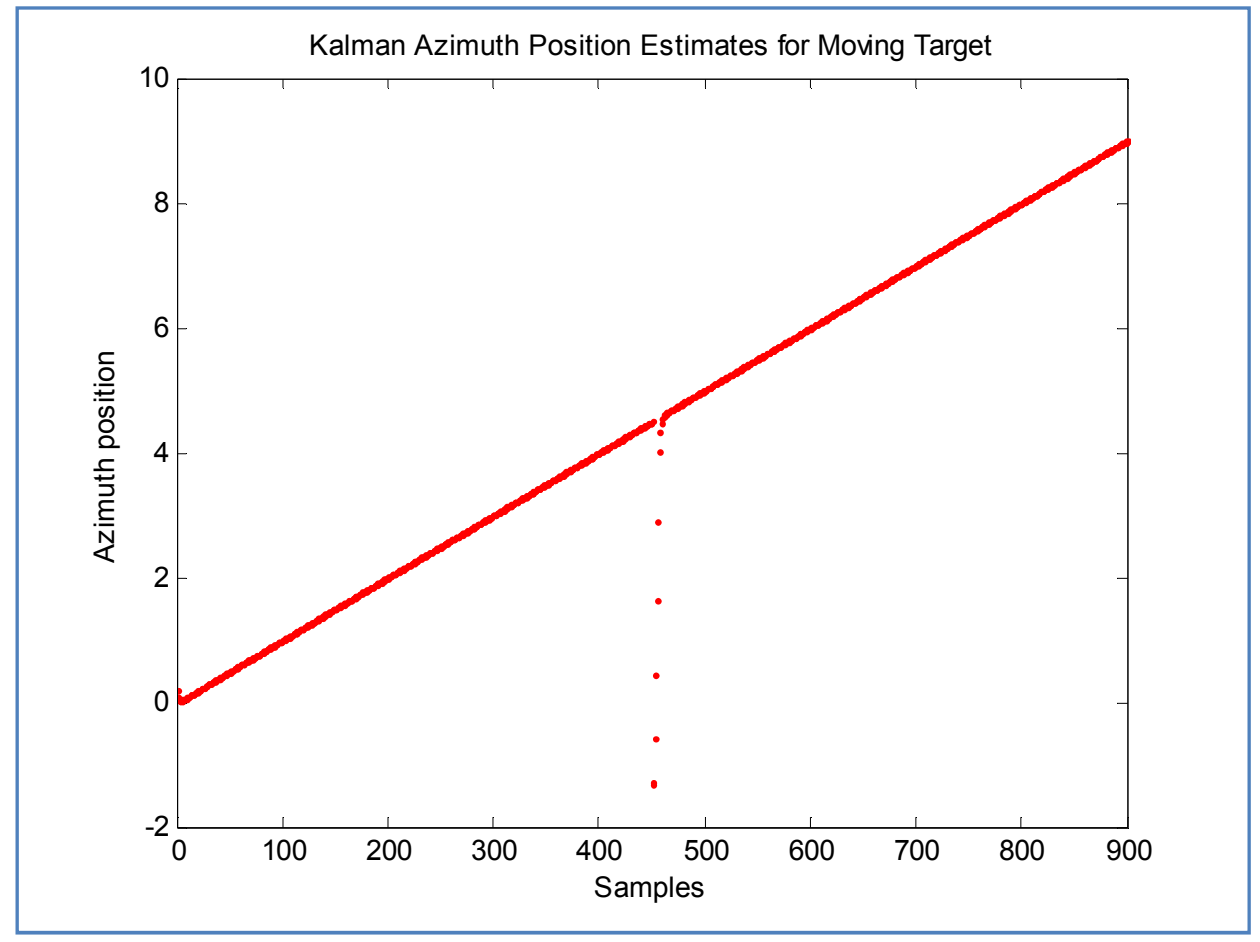

Figure 6.6: Kalman Azimuth Position Estimates for Moving 2-D Target

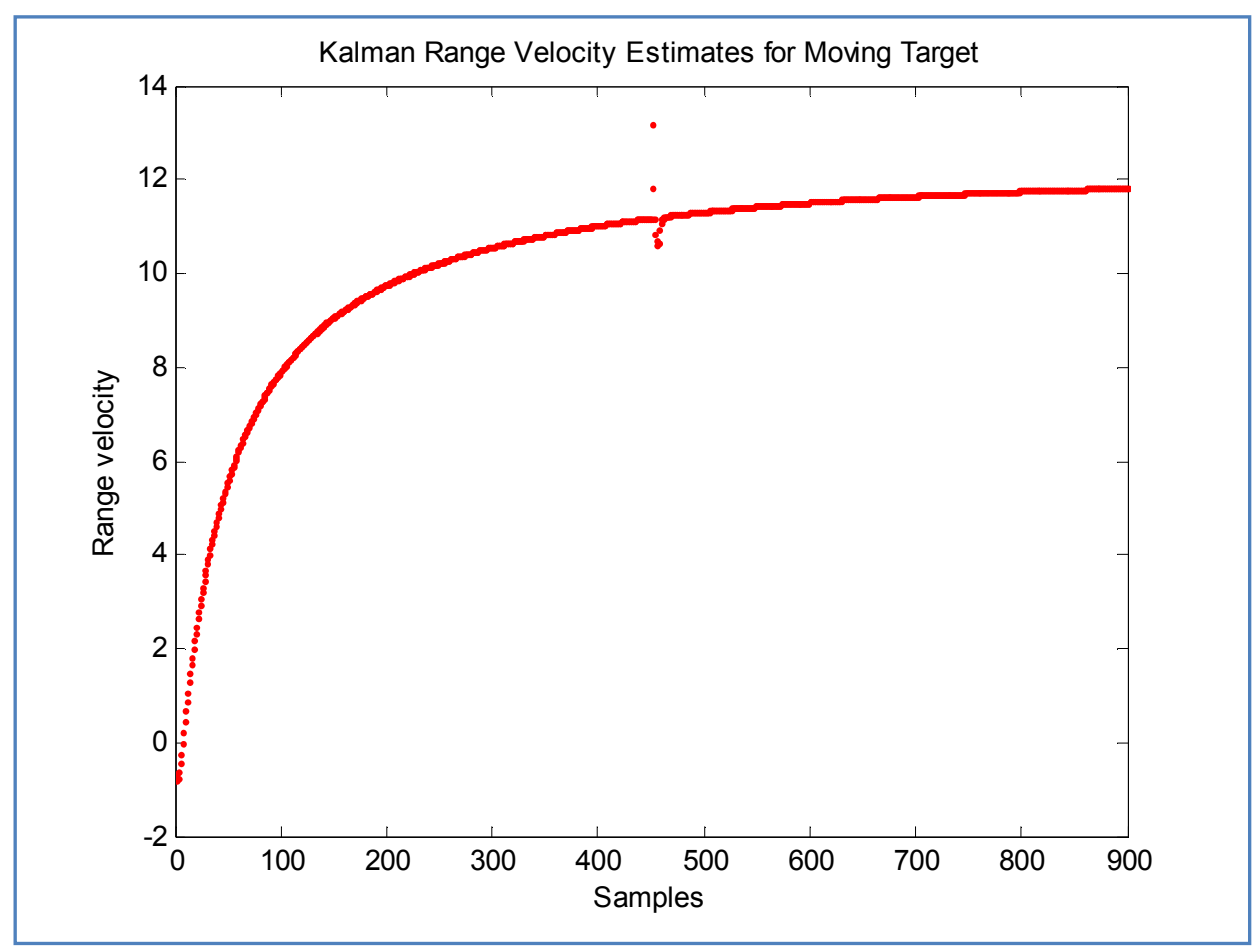

Figure 6.7: Kalman Range Velocity Estimates for Moving 2-D Target 


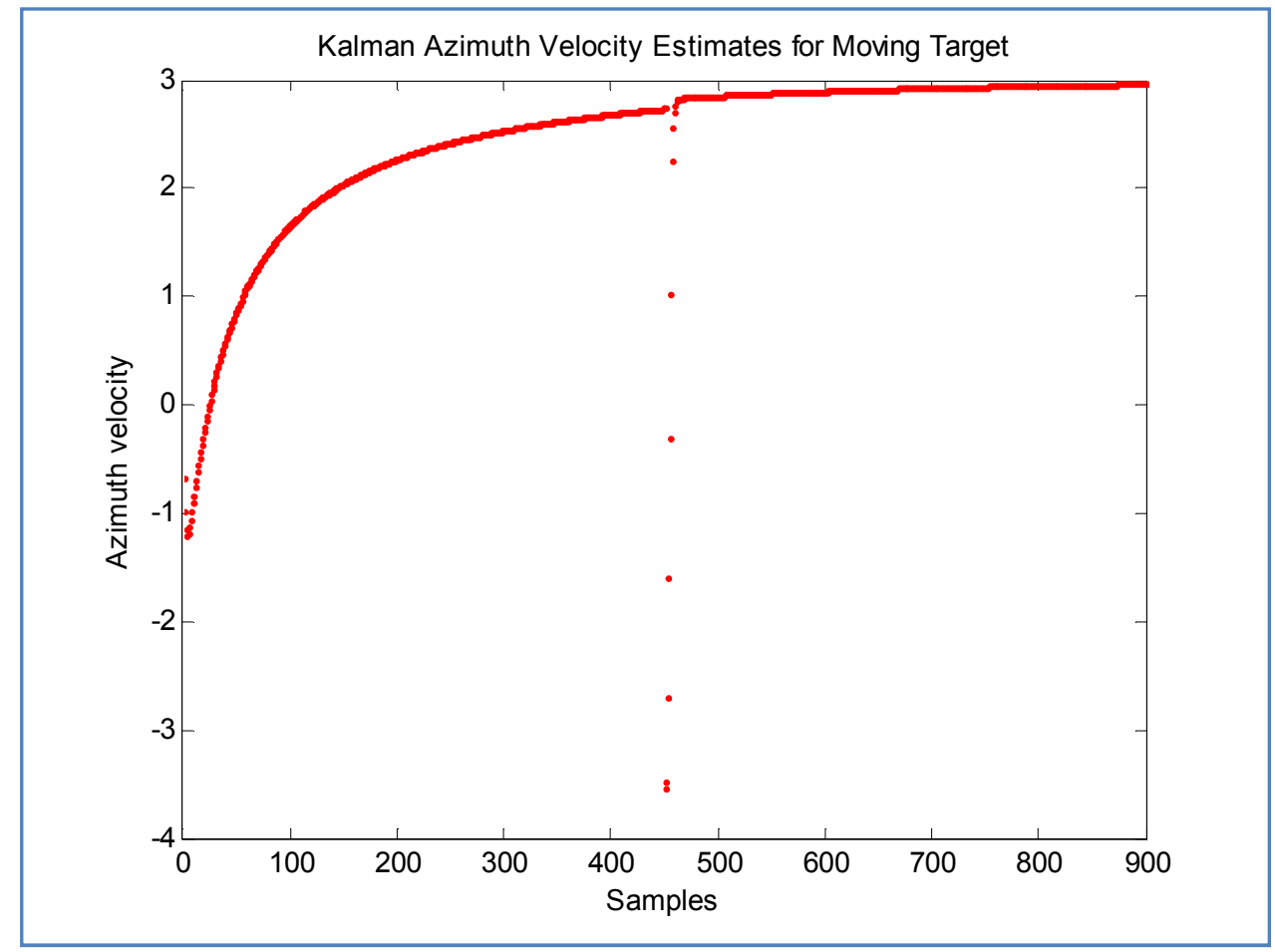

Figure 6.8: Kalman Azimuth Velocity Estimates for Moving 2-D Target

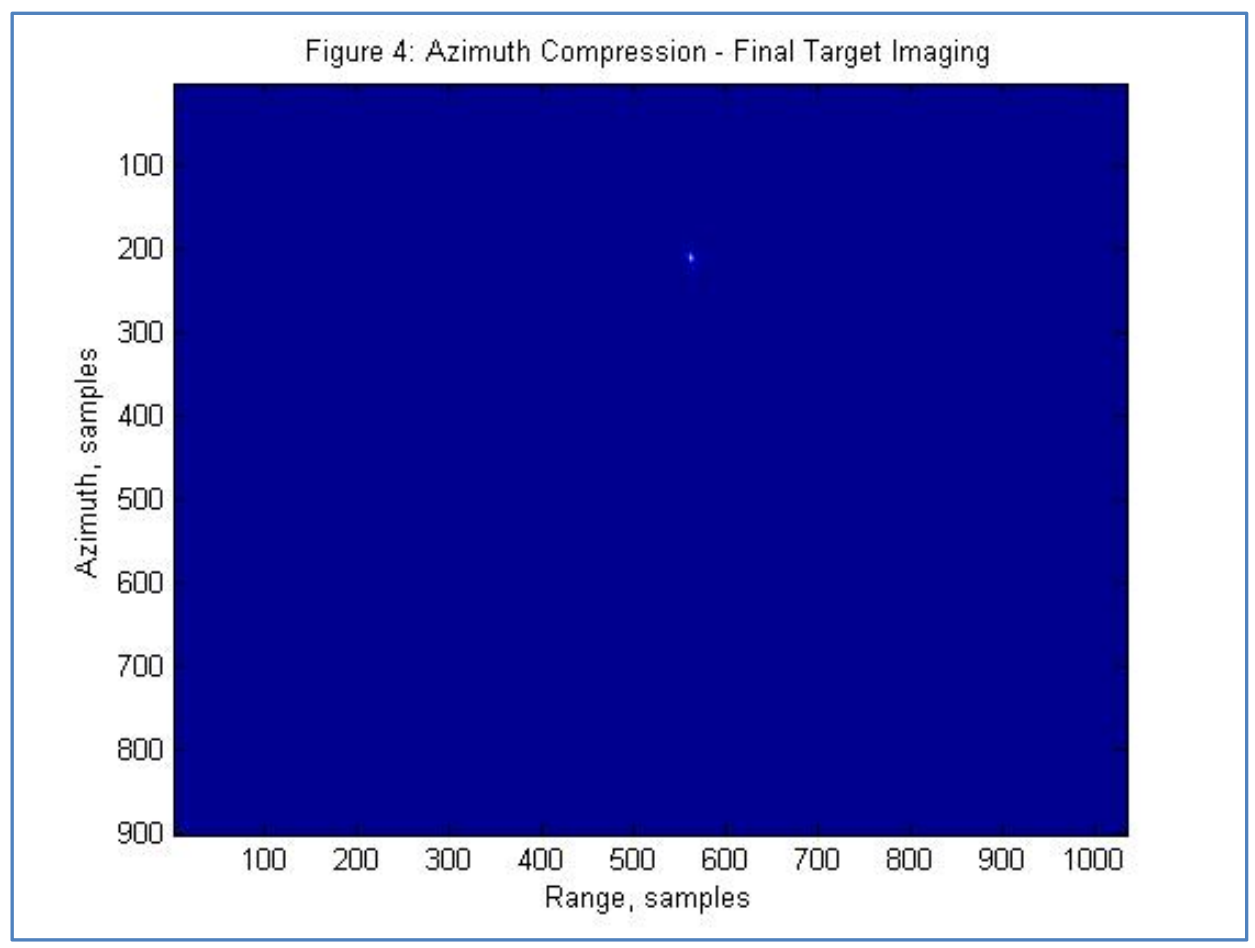

Figure 6.9: Final Target Imaging for 2-D Moving Target 


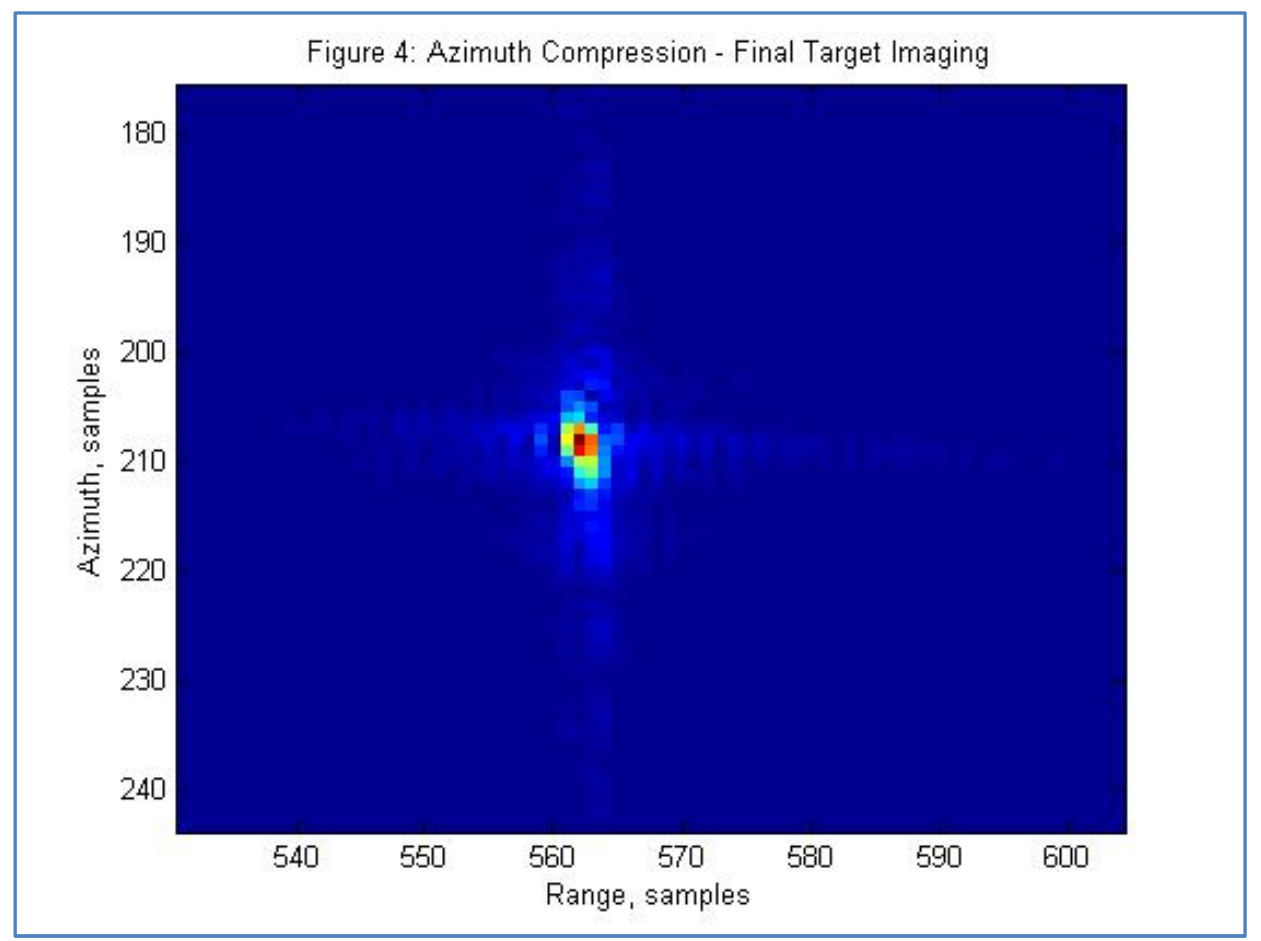

Figure 6.10: Final Target Imaging for 2-D Moving Target -- Zoomed In

The reason for the abrupt change in position and velocity values in Figures 6.5 through 6.8 around 450 samples is because this is the assumed approximate point when the SAR platform passes by the target, thus the signs on the equations change. The Kalman filter corrects for this and the true position and velocity estimates continue to converge.

\subsection{Incorporating Measurement Noise}

To simulate measurements that are realizable in a practical application, it is desirable to add noise to the individual slant range and squint angle measurements. Between iterations of slant range or squint angle, the measurement values change by a very small amount, on the order of $10^{-5}$ meters or radians. For this reason, it is difficult to add noise, since the amount of noise added needs to be even smaller. 
For added noise of 0.0002 meters and 0.00000005 radians, the following results in Figures 6.11 through 6.13 are typical for the Kalman filter for $P$ values of 0.001 to 10 and Q values of 1 to 1000 .
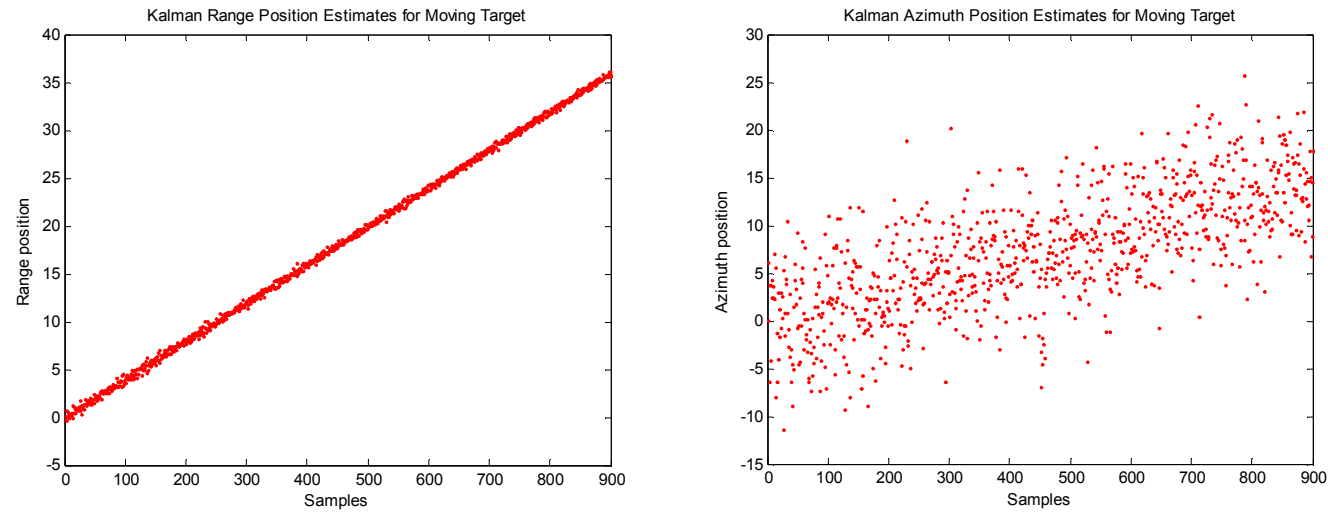

Figure 6.11: Kalman Range and Position Estimations for 2-D Moving Target
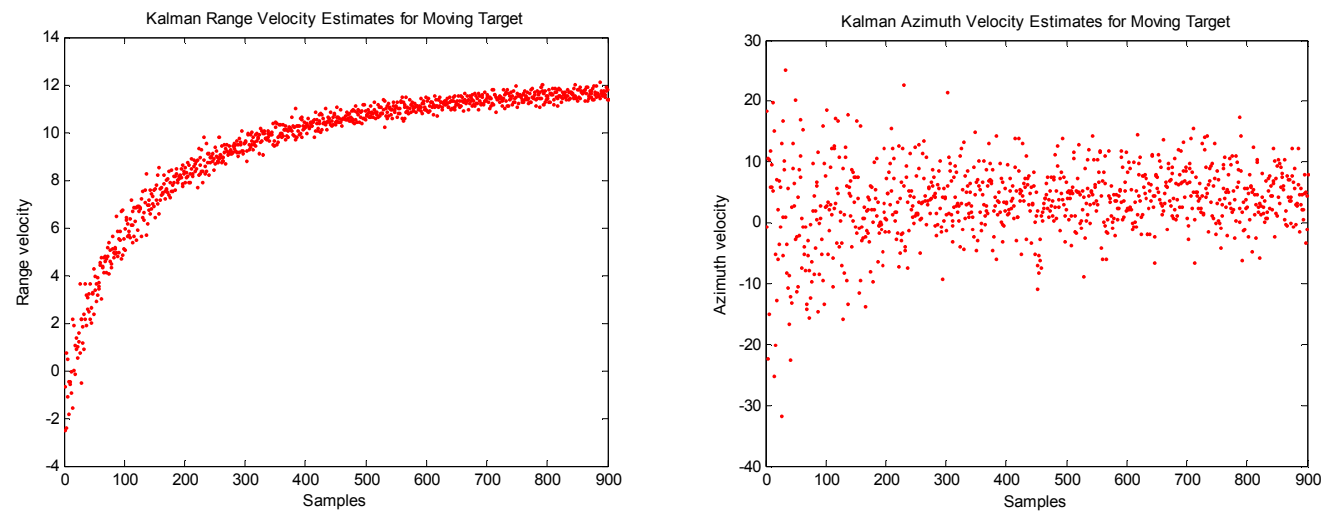

Figure 6.12: Kalman Range and Position Velocity Estimations for 2-D Moving Target 


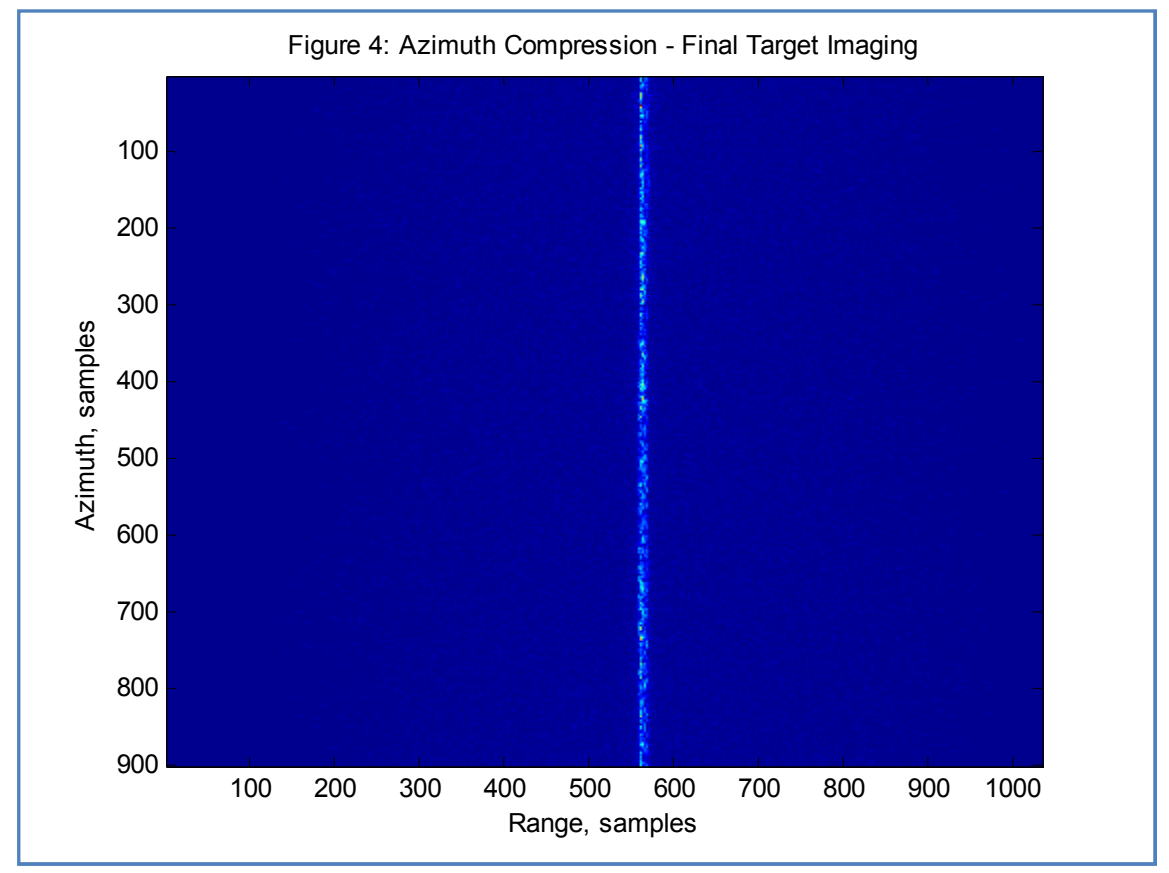

Figure 6.13: Final Target Imaging for 2-D Moving Target with Added Measurement Noise

As can be seen in Figures 6.11 and 6.12, the range direction converges much better than the azimuth direction. For this reason, it is not possible to compress the azimuth direction to determine the final target location. Figure 6.13 shows the final target location. While the range location is constant, the azimuth direction is a line over all space-implying the range Doppler algorithm was unable to correctly image the target with measurement noise added.

\subsection{Slow Time SAR Tracking Using H Infinity}

To compare results with a filter that is not as dependent on noise characteristics, the Kalman filter code can easily be replaced with the same $\mathrm{H}$ Infinity algorithm used earlier in this thesis. Results are then compared to the Kalman filter results using mean-squared errors as metric (between the filter estimates and the "true" target vehicle location). 


\subsubsection{H Infinity Filter Testing}

Using the same noise parameters and same target velocities as were used to test the Kalman filter's response to noisy measurements, the $\mathrm{H}$ Infinity filter was tested as a tracking filter in the slow-time domain. Figure 6.14 shows the range and azimuth estimated positions outputted by the filter.
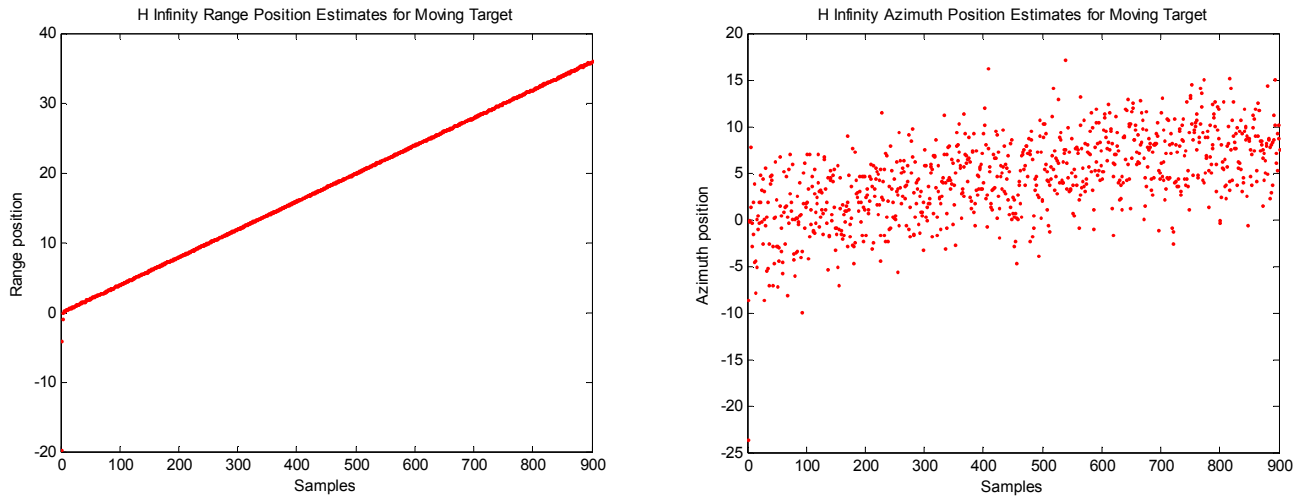

Figure 6.14: H Infinity Tracking Results

The $\mathrm{H}$ Infinity filter was found to produce the same results as the Kalman filter when little or no noise was added to the measurement signals, but yielded much less consistent tracks in the azimuth dimension when the noise was added in. This is most likely due to the nature of the $\mathrm{H}$ Infinity filter, which requires fine-tuning to accommodate new noise situations.

The $\mathrm{H}$ Infinity filter also proved to be more sensitive to changes in the coefficients used to create the $\mathrm{P}$ and $\mathrm{Q}$ noise covariance matrices. Some values of these coefficients caused the output track of the $\mathrm{H}$ Infinity filter to look something like that shown in 
Figure 6.15. While the track itself is relatively accurate, it can be seen in the image that the track jumps out of its current path somewhat periodically. The velocity seems to remain constant, but the absolute position predicted by the filter is somewhat unpredictable. When mean-squared errors are used as a metric to compare the $\mathrm{H}$ Infinity filter to the Kalman filter, the unpredictable nature of the $\mathrm{H}$ Infinity filter becomes even clearer.

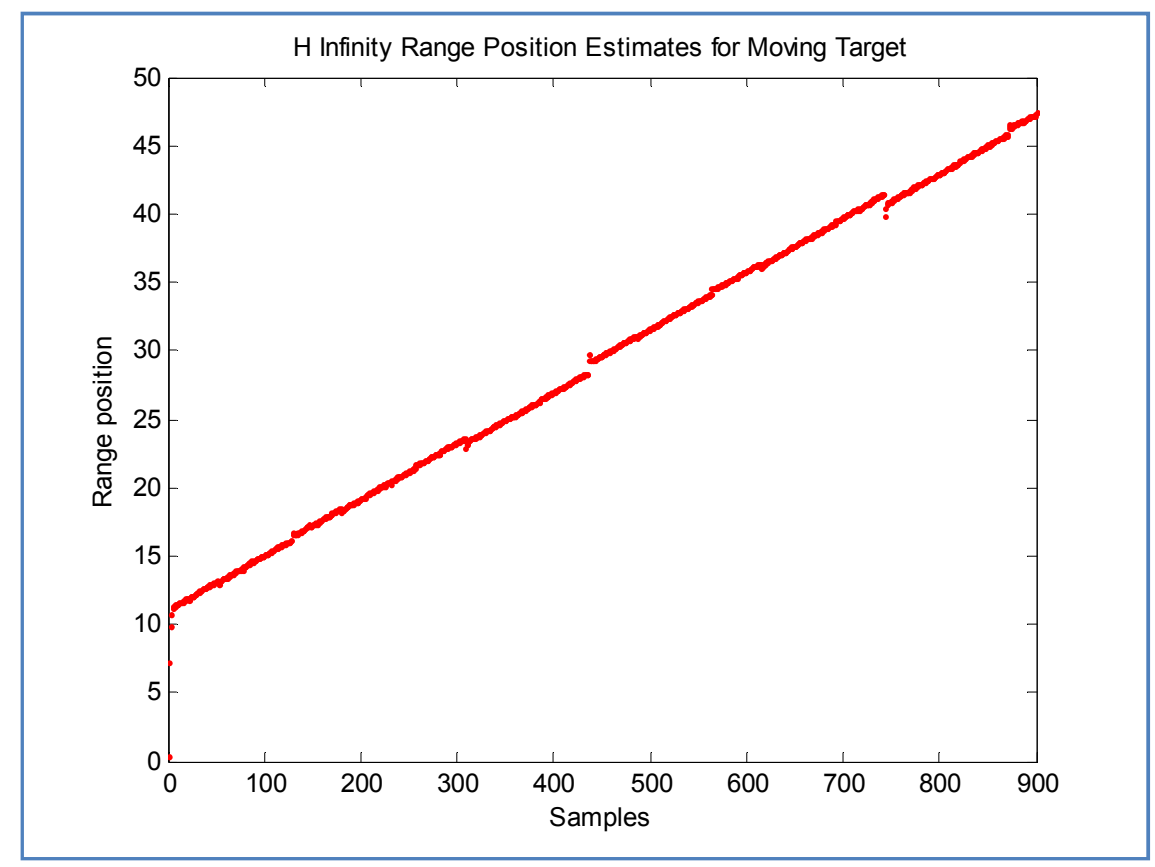

Figure 6.15: Slow-time H Infinity Filter Periodic Track Jumping

\subsubsection{Slow-Time Filter Type Comparison}

The Kalman and $\mathrm{H}$ Infinity filters, as used to track the slow-time position of the targets, were compared to one another using mean-squared errors as a test metric. The error between the position estimates of each filter were compared to the true known point target vehicle positions in both the range and azimuth dimensions. Figure 6.16 below 
shows a sample table that illustrates the mean-squared error performance as the $\mathrm{P}$ and $\mathrm{Q}$ matrices are varied. The amount of measurement noise is constant throughout each iteration. Figures 6.19 and 6.20 show a 3-D example of the data in Figure 6.16. It can be seen that there is no good correlation between $\mathrm{P}$ and $\mathrm{Q}$ matrix values and the outcome of the mean squared error values. The $\mathrm{H}$ Infinity filter performance is more erratic and does not follow any sort of discernible pattern, whereas the Kalman filter performance has a pattern-as $Q$ increases, range mean squared error increases and azimuth mean squared error decreases. 


\begin{tabular}{|c|c|c|}
\hline $\begin{array}{l}\text { Slant Range Variance }= \\
0.002 \text { meters } \\
\text { Squint Angle Variance }=0.00000005 \\
\text { radians }\end{array}$ & Kalman & H Infinity \\
\hline $\begin{array}{l}Q=0.001 \\
P=1\end{array}$ & $\begin{array}{c}\text { Range: } 4.75 \\
\text { Azimuth: } 6.79\end{array}$ & $\begin{array}{c}\text { Range: } 13.15 \\
\text { Azimuth: } 23.83\end{array}$ \\
\hline $\begin{array}{l}Q=0.01 \\
P=1\end{array}$ & $\begin{array}{c}\text { Range: } 3.49 \\
\text { Azimuth: } 10.15\end{array}$ & $\begin{array}{c}\text { Range: } 12.84 \\
\text { Azimuth: } 22.76\end{array}$ \\
\hline $\begin{array}{l}Q=0.1 \\
P=1\end{array}$ & $\begin{array}{c}\text { Range: } 1.28 \\
\text { Azimuth: } 14.16\end{array}$ & $\begin{array}{l}\text { Range: } 10.00 \\
\text { Azimuth: } 20.99\end{array}$ \\
\hline $\begin{array}{l}Q=0.5 \\
P=1\end{array}$ & $\begin{array}{c}\text { Range: } 0.316 \\
\text { Azimuth: } 18.28\end{array}$ & $\begin{array}{c}\text { Range: } 0.92 \\
\text { Azimuth: } 26.89\end{array}$ \\
\hline $\begin{array}{l}Q=0.001 \\
P=10\end{array}$ & $\begin{array}{c}\text { Range: } 4.28 \\
\text { Azimuth: } 7.78\end{array}$ & $\begin{array}{c}\text { Range: } 0.41 \\
\text { Azimuth: } 9.72\end{array}$ \\
\hline $\begin{array}{l}Q=0.01 \\
P=10\end{array}$ & $\begin{array}{c}\text { Range: } 3.10 \\
\text { Azimuth: } 9.91\end{array}$ & $\begin{array}{c}\text { Range: } 0.34 \\
\text { Azimuth: } 9.48\end{array}$ \\
\hline $\begin{array}{l}Q=0.1 \\
P=10\end{array}$ & $\begin{array}{c}\text { Range: } 0.96 \\
\text { Azimuth: } 14.64\end{array}$ & $\begin{array}{c}\text { Range: } 0.0064 \\
\text { Azimuth: } 11.05\end{array}$ \\
\hline $\begin{array}{l}Q=0.5 \\
P=10\end{array}$ & $\begin{array}{c}\text { Range: } 0.16 \\
\text { Azimuth: } 18.33\end{array}$ & $\begin{array}{c}\text { Range: } 21.57 \\
\text { Azimuth: } 51.77\end{array}$ \\
\hline $\begin{array}{l}Q=0.001 \\
P=100\end{array}$ & $\begin{array}{c}\text { Range: } 4.31 \\
\text { Azimuth: } 6.99\end{array}$ & $\begin{array}{c}\text { Range: } 0.006 \\
\text { Azimuth: } 9.19\end{array}$ \\
\hline $\begin{array}{l}Q=0.01 \\
P=100\end{array}$ & $\begin{array}{c}\text { Range: } 2.98 \\
\text { Azimuth: } 9.49\end{array}$ & $\begin{array}{c}\text { Range: } 251.48 \\
\text { Azimuth: } 279.95\end{array}$ \\
\hline $\begin{array}{l}Q=0.1 \\
P=100\end{array}$ & $\begin{array}{c}\text { Range: } 0.95 \\
\text { Azimuth: } 14.4\end{array}$ & $\begin{array}{c}\text { Range: } 0.47 \\
\text { Azimuth: } 10.88\end{array}$ \\
\hline $\begin{array}{l}Q=0.5 \\
P=100\end{array}$ & $\begin{array}{c}\text { Range: } 0.164 \\
\text { Azimuth: } 18.51\end{array}$ & $\begin{array}{c}\text { Range: } 43.99 \\
\text { Azimuth: } 73.51\end{array}$ \\
\hline $\begin{array}{l}Q=0.001 \\
P=1000\end{array}$ & $\begin{array}{c}\text { Range: } 4.29 \\
\text { Azimuth: } 6.92\end{array}$ & $\begin{array}{c}\text { Range: } 15198 \\
\text { Azimuth: } 16822\end{array}$ \\
\hline $\begin{array}{l}Q=0.01 \\
P=1000\end{array}$ & $\begin{array}{c}\text { Range: } 3.03 \\
\text { Azimuth: } 9.32\end{array}$ & $\begin{array}{l}\text { Range: } 0.0061 \\
\text { Azimuth: } 9.05\end{array}$ \\
\hline $\begin{array}{l}Q=0.1 \\
P=1000\end{array}$ & $\begin{array}{c}\text { Range: } 0.97 \\
\text { Azimuth: } 14.63\end{array}$ & $\begin{array}{c}\text { Range: } 0.58 \\
\text { Azimuth: } 11.23\end{array}$ \\
\hline $\begin{array}{l}Q=0.5 \\
P=1000\end{array}$ & $\begin{array}{c}\text { Range: } 0.17 \\
\text { Azimuth: } 18.95\end{array}$ & $\begin{array}{c}\text { Range: } 47.41 \\
\text { Azimuth: } 76.13\end{array}$ \\
\hline
\end{tabular}

Figure 6.16: Mean-Squared Error Performance of Kalman and H Infinity Filters 


\begin{tabular}{|c|c|c|}
\hline $\begin{array}{l}\text { Slant Range Variance }= \\
0.002 \text { meters } \\
\text { Squint Angle Variance }=0.00000005 \\
\text { radians }\end{array}$ & Kalman & H Infinity \\
\hline $\begin{array}{l}Q=0.001 \\
P=1\end{array}$ & $\begin{array}{c}\text { Range: } 4.75 \\
\text { Azimuth: } 6.79\end{array}$ & $\begin{array}{c}\text { Range: } 13.15 \\
\text { Azimuth: } 23.83\end{array}$ \\
\hline $\begin{array}{l}Q=0.01 \\
P=1\end{array}$ & $\begin{array}{c}\text { Range: } 3.49 \\
\text { Azimuth: } 10.15\end{array}$ & $\begin{array}{c}\text { Range: } 12.84 \\
\text { Azimuth: } 22.76\end{array}$ \\
\hline $\begin{array}{l}Q=0.1 \\
P=1\end{array}$ & $\begin{array}{c}\text { Range: } 1.28 \\
\text { Azimuth: } 14.16\end{array}$ & $\begin{array}{l}\text { Range: } 10.00 \\
\text { Azimuth: } 20.99\end{array}$ \\
\hline $\begin{array}{l}Q=0.5 \\
P=1\end{array}$ & $\begin{array}{c}\text { Range: } 0.316 \\
\text { Azimuth: } 18.28\end{array}$ & $\begin{array}{c}\text { Range: } 0.92 \\
\text { Azimuth: } 26.89\end{array}$ \\
\hline $\begin{array}{l}Q=0.001 \\
P=10\end{array}$ & $\begin{array}{c}\text { Range: } 4.28 \\
\text { Azimuth: } 7.78\end{array}$ & $\begin{array}{c}\text { Range: } 0.41 \\
\text { Azimuth: } 9.72\end{array}$ \\
\hline $\begin{array}{l}Q=0.01 \\
P=10\end{array}$ & $\begin{array}{c}\text { Range: } 3.10 \\
\text { Azimuth: } 9.91\end{array}$ & $\begin{array}{c}\text { Range: } 0.34 \\
\text { Azimuth: } 9.48\end{array}$ \\
\hline $\begin{array}{l}Q=0.1 \\
P=10\end{array}$ & $\begin{array}{c}\text { Range: } 0.96 \\
\text { Azimuth: } 14.64\end{array}$ & $\begin{array}{c}\text { Range: } 0.0064 \\
\text { Azimuth: } 11.05\end{array}$ \\
\hline $\begin{array}{l}Q=0.5 \\
P=10\end{array}$ & $\begin{array}{c}\text { Range: } 0.16 \\
\text { Azimuth: } 18.33\end{array}$ & $\begin{array}{c}\text { Range: } 21.57 \\
\text { Azimuth: } 51.77\end{array}$ \\
\hline $\begin{array}{l}Q=0.001 \\
P=100\end{array}$ & $\begin{array}{c}\text { Range: } 4.31 \\
\text { Azimuth: } 6.99\end{array}$ & $\begin{array}{c}\text { Range: } 0.006 \\
\text { Azimuth: } 9.19\end{array}$ \\
\hline $\begin{array}{l}Q=0.01 \\
P=100\end{array}$ & $\begin{array}{c}\text { Range: } 2.98 \\
\text { Azimuth: } 9.49\end{array}$ & $\begin{array}{c}\text { Range: } 251.48 \\
\text { Azimuth: } 279.95\end{array}$ \\
\hline $\begin{array}{l}Q=0.1 \\
P=100\end{array}$ & $\begin{array}{c}\text { Range: } 0.95 \\
\text { Azimuth: } 14.4\end{array}$ & $\begin{array}{c}\text { Range: } 0.47 \\
\text { Azimuth: } 10.88\end{array}$ \\
\hline $\begin{array}{l}Q=0.5 \\
P=100\end{array}$ & $\begin{array}{c}\text { Range: } 0.164 \\
\text { Azimuth: } 18.51\end{array}$ & $\begin{array}{c}\text { Range: } 43.99 \\
\text { Azimuth: } 73.51\end{array}$ \\
\hline $\begin{array}{l}Q=0.001 \\
P=1000\end{array}$ & $\begin{array}{c}\text { Range: } 4.29 \\
\text { Azimuth: } 6.92\end{array}$ & $\begin{array}{c}\text { Range: } 15198 \\
\text { Azimuth: } 16822\end{array}$ \\
\hline $\begin{array}{l}Q=0.01 \\
P=1000\end{array}$ & $\begin{array}{c}\text { Range: } 3.03 \\
\text { Azimuth: } 9.32\end{array}$ & $\begin{array}{l}\text { Range: } 0.0061 \\
\text { Azimuth: } 9.05\end{array}$ \\
\hline $\begin{array}{l}Q=0.1 \\
P=1000\end{array}$ & $\begin{array}{c}\text { Range: } 0.97 \\
\text { Azimuth: } 14.63\end{array}$ & $\begin{array}{c}\text { Range: } 0.58 \\
\text { Azimuth: } 11.23\end{array}$ \\
\hline $\begin{array}{l}Q=0.5 \\
P=1000\end{array}$ & $\begin{array}{c}\text { Range: } 0.17 \\
\text { Azimuth: } 18.95\end{array}$ & $\begin{array}{c}\text { Range: } 47.41 \\
\text { Azimuth: } 76.13\end{array}$ \\
\hline
\end{tabular}

Figure 6.17: Mean-Squared Error Performance of Kalman and H Infinity Filters 


\begin{tabular}{|c|c|c|}
\hline $\begin{array}{l}\text { Slant Range Variance }= \\
0.002 \text { meters } \\
\text { Squint Angle Variance }=0.00000005 \\
\text { radians }\end{array}$ & Kalman & H Infinity \\
\hline $\begin{array}{l}Q=0.001 \\
P=1\end{array}$ & $\begin{array}{c}\text { Range: } 4.75 \\
\text { Azimuth: } 6.79\end{array}$ & $\begin{array}{c}\text { Range: } 13.15 \\
\text { Azimuth: } 23.83\end{array}$ \\
\hline $\begin{array}{l}Q=0.01 \\
P=1\end{array}$ & $\begin{array}{c}\text { Range: } 3.49 \\
\text { Azimuth: } 10.15\end{array}$ & $\begin{array}{c}\text { Range: } 12.84 \\
\text { Azimuth: } 22.76\end{array}$ \\
\hline $\begin{array}{l}Q=0.1 \\
P=1\end{array}$ & $\begin{array}{c}\text { Range: } 1.28 \\
\text { Azimuth: } 14.16\end{array}$ & $\begin{array}{l}\text { Range: } 10.00 \\
\text { Azimuth: } 20.99\end{array}$ \\
\hline $\begin{array}{l}Q=0.5 \\
P=1\end{array}$ & $\begin{array}{c}\text { Range: } 0.316 \\
\text { Azimuth: } 18.28\end{array}$ & $\begin{array}{c}\text { Range: } 0.92 \\
\text { Azimuth: } 26.89\end{array}$ \\
\hline $\begin{array}{l}Q=0.001 \\
P=10\end{array}$ & $\begin{array}{c}\text { Range: } 4.28 \\
\text { Azimuth: } 7.78\end{array}$ & $\begin{array}{c}\text { Range: } 0.41 \\
\text { Azimuth: } 9.72\end{array}$ \\
\hline $\begin{array}{l}Q=0.01 \\
P=10\end{array}$ & $\begin{array}{c}\text { Range: } 3.10 \\
\text { Azimuth: } 9.91\end{array}$ & $\begin{array}{c}\text { Range: } 0.34 \\
\text { Azimuth: } 9.48\end{array}$ \\
\hline $\begin{array}{l}Q=0.1 \\
P=10\end{array}$ & $\begin{array}{c}\text { Range: } 0.96 \\
\text { Azimuth: } 14.64\end{array}$ & $\begin{array}{c}\text { Range: } 0.0064 \\
\text { Azimuth: } 11.05\end{array}$ \\
\hline $\begin{array}{l}Q=0.5 \\
P=10\end{array}$ & $\begin{array}{c}\text { Range: } 0.16 \\
\text { Azimuth: } 18.33\end{array}$ & $\begin{array}{c}\text { Range: } 21.57 \\
\text { Azimuth: } 51.77\end{array}$ \\
\hline $\begin{array}{l}Q=0.001 \\
P=100\end{array}$ & $\begin{array}{c}\text { Range: } 4.31 \\
\text { Azimuth: } 6.99\end{array}$ & $\begin{array}{c}\text { Range: } 0.006 \\
\text { Azimuth: } 9.19\end{array}$ \\
\hline $\begin{array}{l}Q=0.01 \\
P=100\end{array}$ & $\begin{array}{c}\text { Range: } 2.98 \\
\text { Azimuth: } 9.49\end{array}$ & $\begin{array}{c}\text { Range: } 251.48 \\
\text { Azimuth: } 279.95\end{array}$ \\
\hline $\begin{array}{l}Q=0.1 \\
P=100\end{array}$ & $\begin{array}{c}\text { Range: } 0.95 \\
\text { Azimuth: } 14.4\end{array}$ & $\begin{array}{c}\text { Range: } 0.47 \\
\text { Azimuth: } 10.88\end{array}$ \\
\hline $\begin{array}{l}Q=0.5 \\
P=100\end{array}$ & $\begin{array}{c}\text { Range: } 0.164 \\
\text { Azimuth: } 18.51\end{array}$ & $\begin{array}{c}\text { Range: } 43.99 \\
\text { Azimuth: } 73.51\end{array}$ \\
\hline $\begin{array}{l}Q=0.001 \\
P=1000\end{array}$ & $\begin{array}{c}\text { Range: } 4.29 \\
\text { Azimuth: } 6.92\end{array}$ & $\begin{array}{c}\text { Range: } 15198 \\
\text { Azimuth: } 16822\end{array}$ \\
\hline $\begin{array}{l}Q=0.01 \\
P=1000\end{array}$ & $\begin{array}{c}\text { Range: } 3.03 \\
\text { Azimuth: } 9.32\end{array}$ & $\begin{array}{l}\text { Range: } 0.0061 \\
\text { Azimuth: } 9.05\end{array}$ \\
\hline $\begin{array}{l}Q=0.1 \\
P=1000\end{array}$ & $\begin{array}{c}\text { Range: } 0.97 \\
\text { Azimuth: } 14.63\end{array}$ & $\begin{array}{c}\text { Range: } 0.58 \\
\text { Azimuth: } 11.23\end{array}$ \\
\hline $\begin{array}{l}Q=0.5 \\
P=1000\end{array}$ & $\begin{array}{c}\text { Range: } 0.17 \\
\text { Azimuth: } 18.95\end{array}$ & $\begin{array}{c}\text { Range: } 47.41 \\
\text { Azimuth: } 76.13\end{array}$ \\
\hline
\end{tabular}

Figure 6.18: Mean-Squared Error Performance of Kalman and H Infinity Filters 


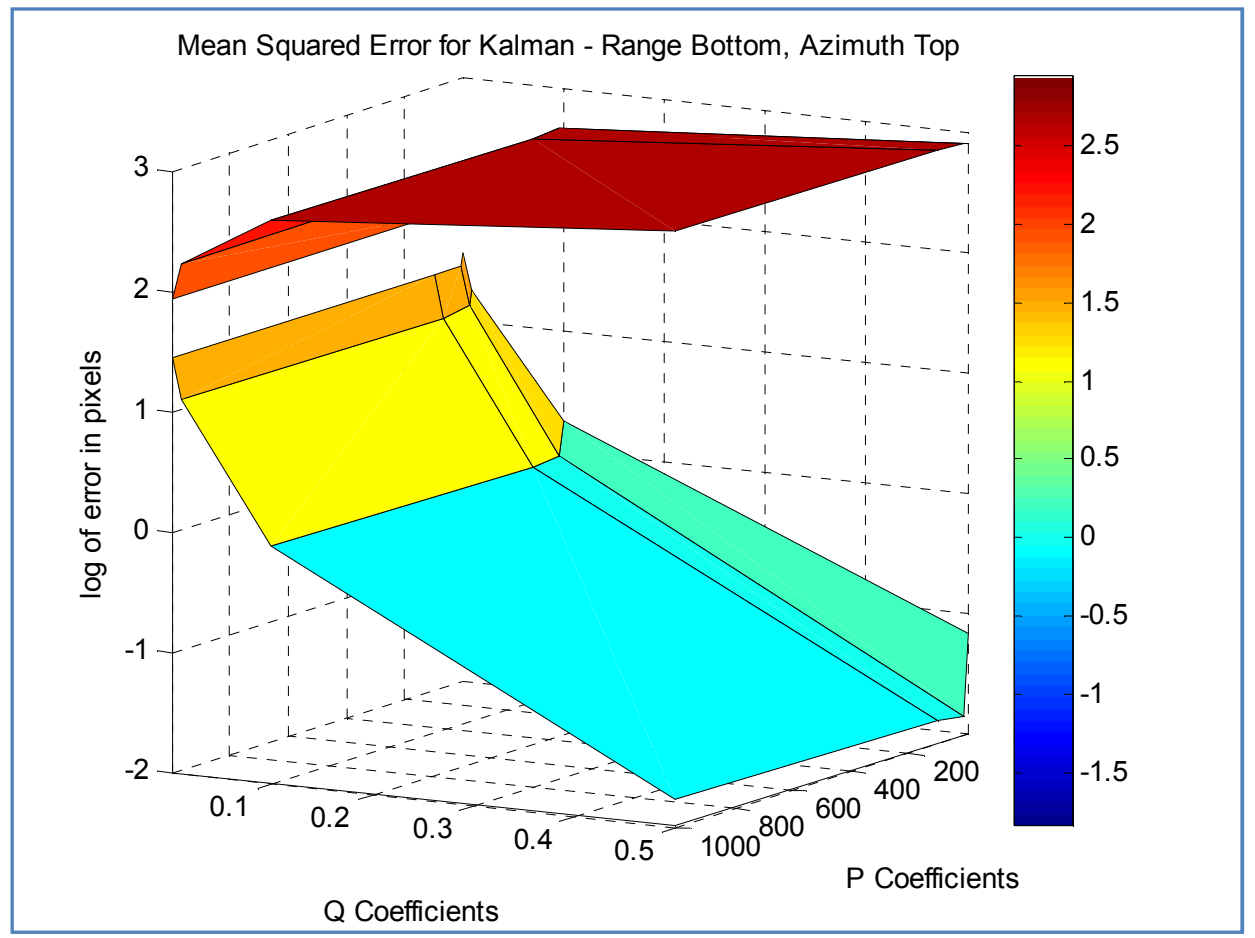

Figure 6.19: Sample Mean Squared Error for Kalman Slow-Time Filter Performance

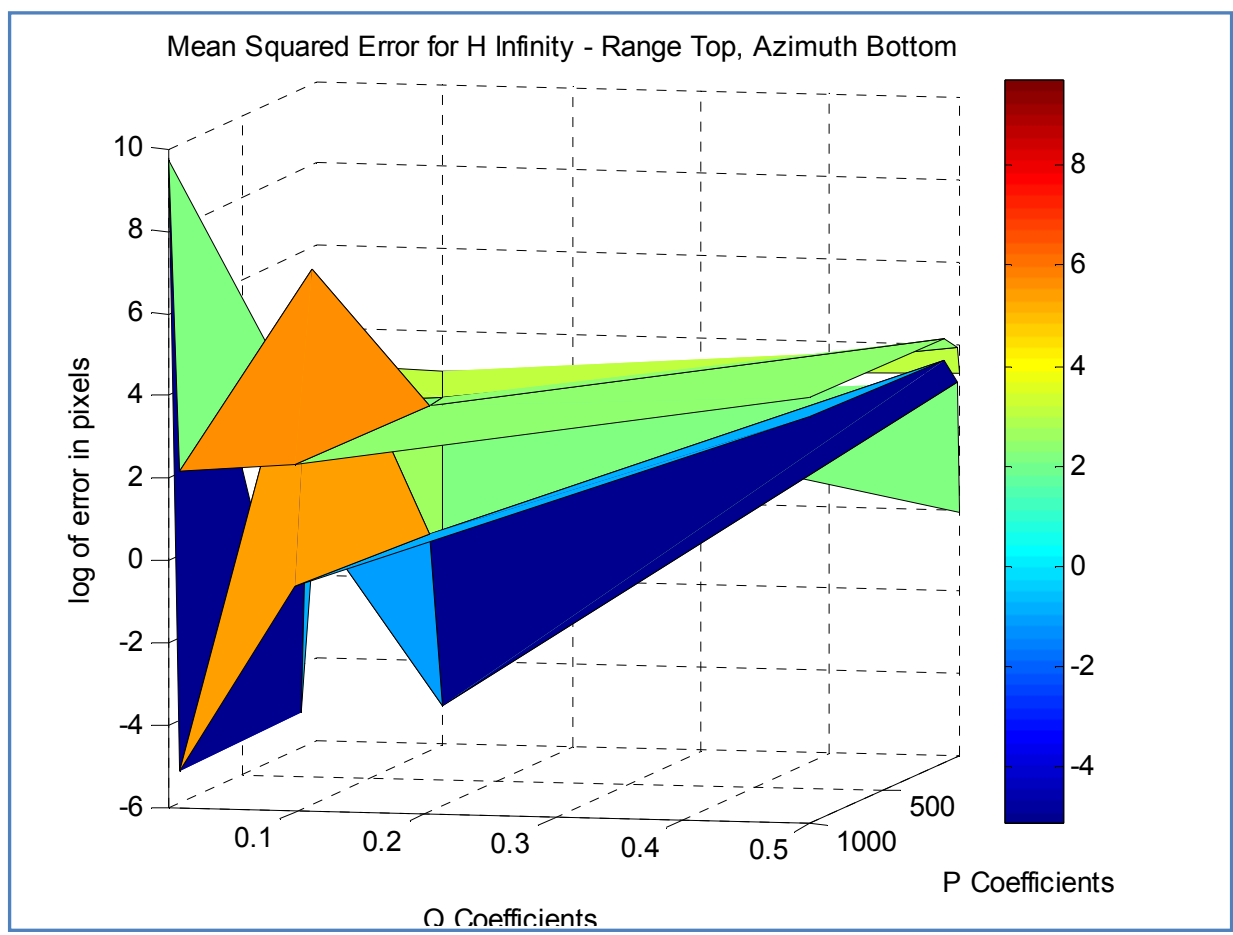

Figure 6.20: Mean Squared Error for H Infinity Slow-Time Filter Performance 


\section{Conclusions and Future Work}

\subsection{Extended Target Tracking in SAR Imagery}

Two primary filter types were investigated in this thesis for the purpose of tracking extended targets throughout several frames of a multi-SAR-image sequence. To prove invariance of the measurement technique used with both filters, the extended target test sets were created to use targets with varying rotations and noise situations. The Kalman and $\mathrm{H}$ Infinity filters proved to be accurate in tracking extended throughout a sequence of SAR frames with mean-squared errors less than 1 pixel for both filters for noise levels of $10 \%$ or less. The two filter types were compared against several filtering parameters using Monte Carlo analysis and were found to be relatively comparable in terms of quality, depending on the noise situation in the imagery. This Monte Carlo method of filter analysis is very useful for determining optimal tracking filter parameters and applications.

The tracking of extended targets throughout a sequence of post-processed SAR images could become useful in future SAR applications as the techniques for the generation of SAR imagery become faster and more efficient with improvements in computing technology. Target tracking in general is very useful in Automatic Target Recognition (ATR) applications due to the possible need for additional images in order to accurately classify a target. If a target can be accurately tracked throughout multiple SAR images it becomes an easier task to classify the target. 


\subsection{Point Target Tracking in SAR Imagery}

The need for additional filter types in the tracking of point targets in sequences of SAR images was shown by the gradual reduction in size of extended targets. The relative size of these point targets was incrementally reduced and the tracked using the Kalman and $\mathrm{H}$ Infinity filters until the target was too small to be tracked these filter types and the same measurement technique that was used for extended target tracking. These filters might still prove useful for this kind of point target tracking if other techniques for instantaneous measurement acquisition could be devised.

The Prediction and Matching Detection (PAMD) Filter and algorithm was then tested for the purpose of circumventing the problems that arose with using Kalman and $\mathrm{H}$ Infinity to track SAR point targets. This filter did successfully track point targets with limited variation of motion, but was shown to be too sensitive to changes in the target vehicle dynamics to be truly useful in a generic SAR tracking application. Despite its shortcomings, the filter did prove to be resilient enough to be used to track multiple targets simultaneously.

The technique that the PAMD Filter implemented for predicting the position of the target vehicle in future image frames could prove useful in future studies if it could be incorporated into the measurement process of the Kalman or $\mathrm{H}$ Infinity filters. Put simply, a technique could be devised for limiting the range of possible measurements for Kalman and $\mathrm{H}$ Infinity, thus combining the principles of the PAMD Filter and the classical filter methods. 


\subsection{Point Target Tracking During Raw SAR Data Collection}

To round out the research topics for SAR target tracking, both the Kalman and H Infinity filters were used to track a SAR point target in slow-time with target movement during the image generation process. The techniques that Brian Zaharris first implemented for tracking in the short-time domain were improved upon to allow for target tracking in both range and azimuth domains simultaneously, as well as for the use of multiple filter types. Zaharris' techniques for the instantaneous measurement of target location were extended to utilize two SAR parameters as raw measurement input, and the robustness of this technique was improved upon by the addition of noise to the signals.

In this application, the Kalman filter did prove to be more robust than the $\mathrm{H}$ Infinity filter, perhaps due to the Kalman filter designer's ability to directly account for relative amount of noise in each dimension. The $\mathrm{H}$ Infinity, on the other hand, often requires a large amount of manual fine-tuning, and this is a likely cause of its slightly weaker performance. In this application, given the technique for measurement, the amount of noise and variation is significantly greater in one dimension (azimuth) than it is in the other dimension, and this fact showed to have an effect on the results of both filters, but in particular on $\mathrm{H}$ Infinity.

\subsubsection{Incorporation with Multi-Image Tracking}

The two generic types of tracking explored during this thesis involved tracking in the short-time domain during the raw data collection process for an individual SAR image as 
well as tracking in real-time throughout the period in which multiple SAR images (and therefore a complete sequence) could be generated. It would be very useful in the context of these applications to be able to accurately connect the two tracking domains, and effectively "pass off" the tracking of one or more point targets in the short-time domain of one image generation to the short-time domain of another image generation, or perhaps another SAR imaging platform altogether.

This connection between the two tracking domains could be useful if a particular target is determined to be of particular interest and must be tracked and accurately imaged for a significant amount of time or using multiple aerial platform vehicles.

\subsubsection{Non-Constant Movement during Raw Data Collection}

It would be quite easy (and useful) to extend the short-time tracking to allow for nonconstant velocities of the tracked targets. The Kalman and $\mathrm{H}$ Infinity filters, as they are currently implemented, should be able to adjust easily with regards to tracking the targets, but simulating motion in this manner may prove difficult given how the simulation is currently being performed. Nevertheless, it would be useful to explore how the technique for target position measurement fares against a target with a nonconstant velocity.

\subsubsection{Incorporation with Automatic Target Recognition}

The various tracking algorithms and techniques, through both short-time data collection and throughout a complete SAR image sequence, could be directly incorporated with 
the target class estimates generated using an ATR algorithm. Especially in the situation where multiple targets are visible in the target region, an accurate ATR classification could be very useful in distinguishing the targets and their respective tracks. The extended tracking of an individual target could also allow for additional SAR image collection (and therefore additional inputs to the ATR algorithm) where without tracking the target might otherwise have been lost.

\subsection{Final Thoughts}

In general, the tracking of extended and point targets in Synthetic Aperture Radar is a crucial task in reconnaissance applications and is a topic of heavy research in the field of radar. This thesis explored the use of multiple discrete-time adaptive filters for the purpose of tracking multiple target types in multiple domains with multiple imaging scenarios.

While the filter types investigated in this project did prove useful, there is definitely no single technique that trumps the rest with regards to tracking targets in SAR, and there is considerable work left to be done to accomplish the task of determining the best filtering techniques for all applications. 


\section{Bibliography}

[1] Bishop, Gary and Welch, Greg. An Introduction to the Kalman Filter. Chapel Hill, NC: Department of Computer Science, University of North Carolina at Chapel Hill, 2001.

[2] DePiero, Fred W. "EE525 - Notes on Kalman Filter". Department of Electrical Engineering, Cal Poly, 2008.

[3] Guilas, Chessa F. "Hausdorff Probabilistic Feature Analysis in SAR Image Recognition." 2006.

[4] Hui, Xu and Zhongkang, Sun. On the Automatic Recognition of Point Target in Same Orbit. Pages 40 - 44. IEEE National Aerospace and Electronics Conference, May 1995.

[5] Hupton, John R. "Three Dimensional Target Modeling with Synthetic Aperture Radar." Cal Poly, 2009.

[6] "Engine Speed Monitoring: The Alpha-Beta Filter." 23 December 2008. Microstar Laboratoies. <http://www.mstarlabs.com/control/engspeed.html>.

[7] Qin, Shi-Yin and Zhu, Feng-You. A Detection Algorithm for Maneuvering IR Point Target and Its Performance Evaluation. Pages $1-5$. IEEE/ASME Mechatronics and Embedded Systems Conference, August 2006. 
[8] Reed I., Gagliardi R, and Shao H. Application of Three Dimensional Filtering to Moving Target Detection. Pages 898-905. IEEE/ASME Transactions on Aerospace and Electronic Systems, November 1983.

[9] Simon, Dan. "From Here to Inifinty". Embedded Systems Programming, vol 14, pages $20-32$. October 2001.

[10] Simon, Dan. Optimal State Estimation: Kalman, $\mathrm{H}$-infinity, and other Nonlinear Approaches. New York, NY: John Wiley and Sons, 2006.

[11] Singh, Jai Prakash. Evolution of the Radar Target Tracking Algorithms: A Move Towards Knowledge Based Multi-Sensor Adaptive Processing. Pages 40-43. IEEE Computations Advances in Multi-Sensor Adaptive Processing, December 2005.

[12] Skolnik, Merrill I. Radar Handbook. New York, NY: Mc-Graw Hill, Inc., 1990.

[13] Soumekh, Mehrdad. Synthetic Aperture Radar Signal Processing. New York, NY: John Wiley \& Sons, Inc., 1999.

[14] "Monte Carlo Analysis." 12 July 2008. Wikipedia: The Free Encyclopedia. <http://en.wikipedia.org/wiki/Monte_Carlo_analysis>.

[15] "Radar." 1 November 2008. Wikipedia: The Free Encyclopedia. $<$ http://en.wikipedia.org/wiki/Radar>. 
[16] "Synthetic Aperture Radar." 30 June 2008. Wikipedia: The Free Encyclopedia. $<$ http://en.wikipedia.org/wiki/Synthetic_aperture_radar>.

[17] Zaharris, Brian. "Two-Dimensional Synthetic Aperture Radar Imaging and Moving Target Tracking Using the Range Doppler Algorithm Simulated in MATLAB." Cal Poly, 2006.

[18] Zhang, Bing and Huanzhang, Lu. The Predicting and Matching Detection Algorithm of Moving Point Target in Image Sequences. Pages 1151 - 1154. IEEE International Conference on Neural Networks and Signal Processing, December 2003. 


\title{
Appendices
}

\author{
findBlob.m
}

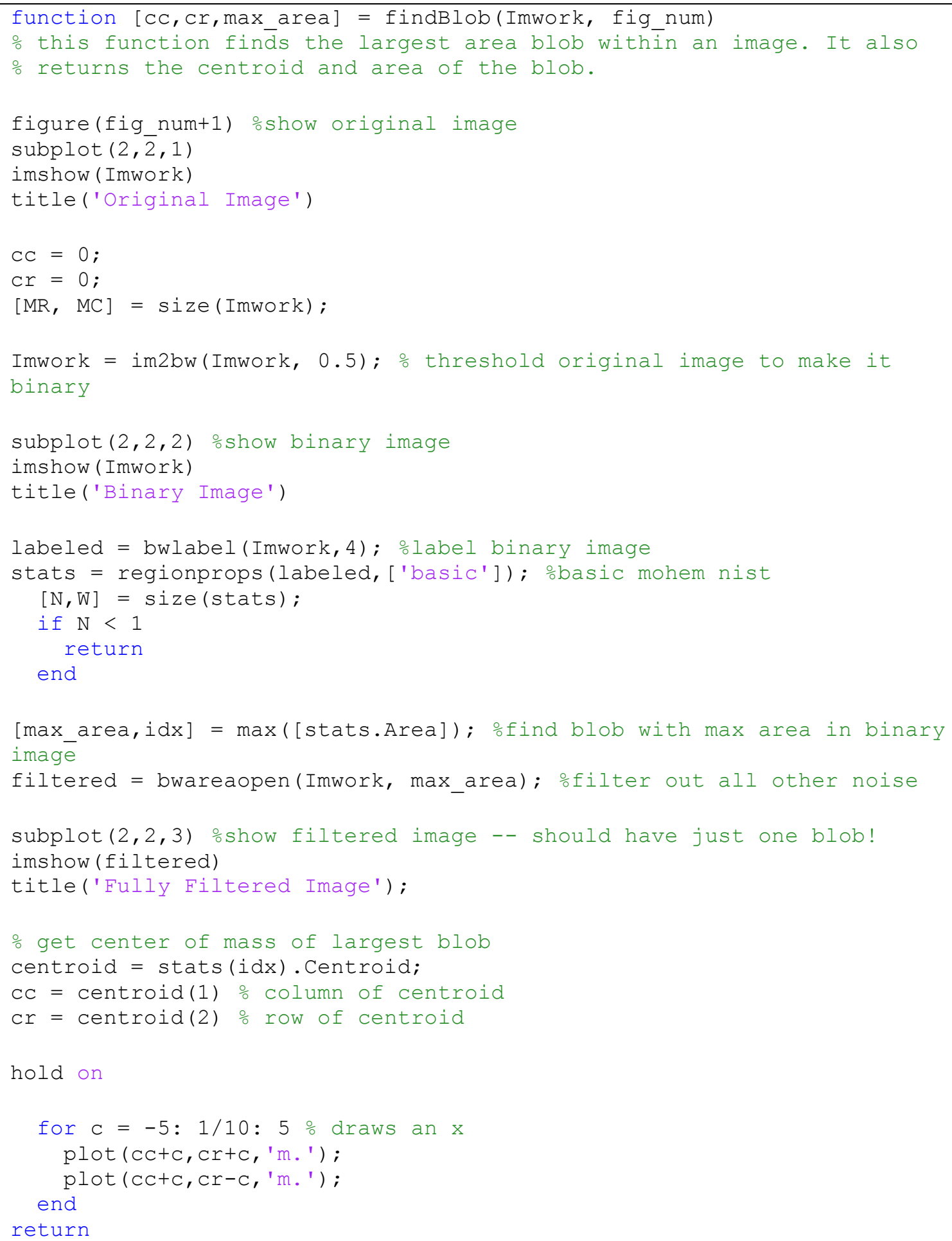




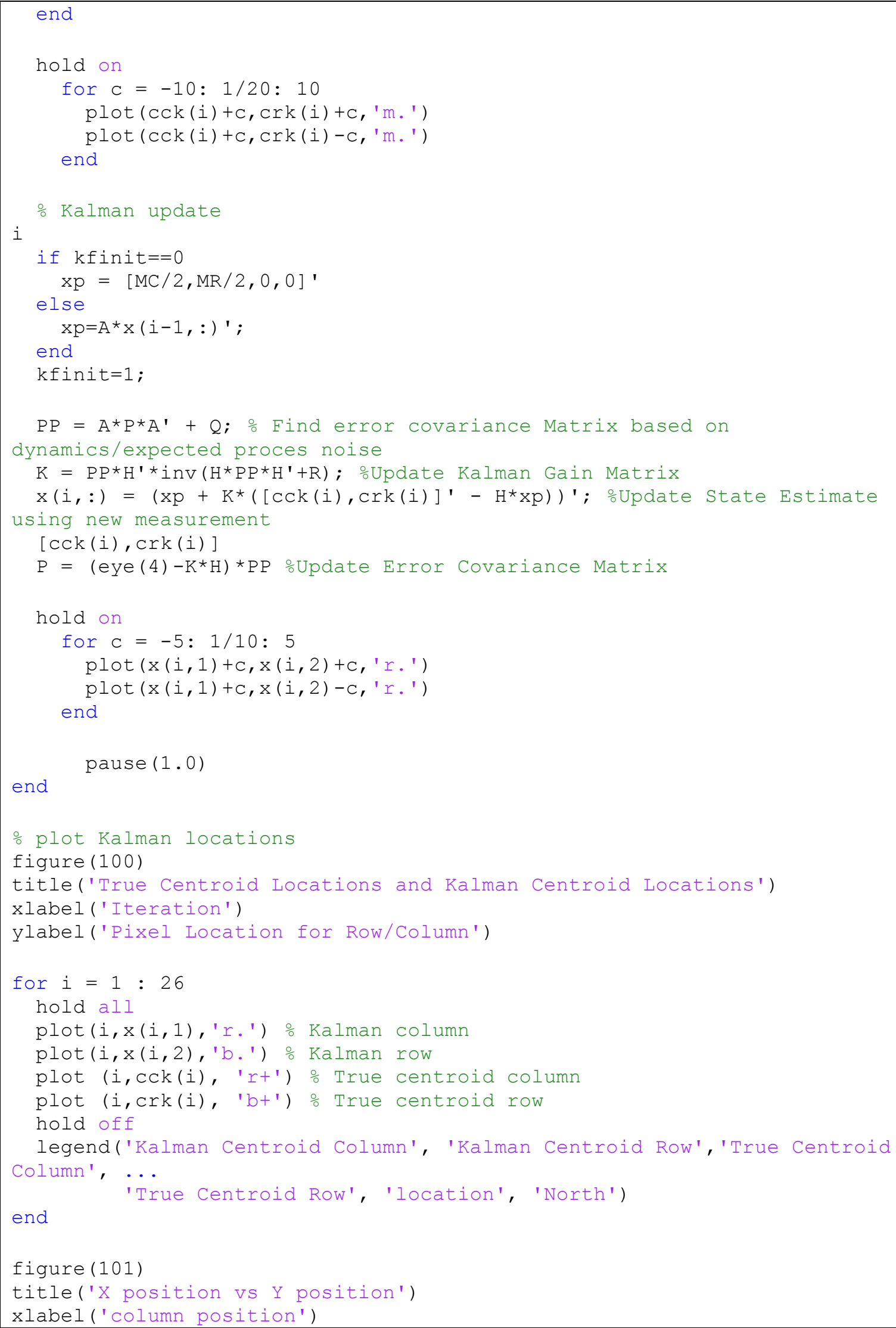




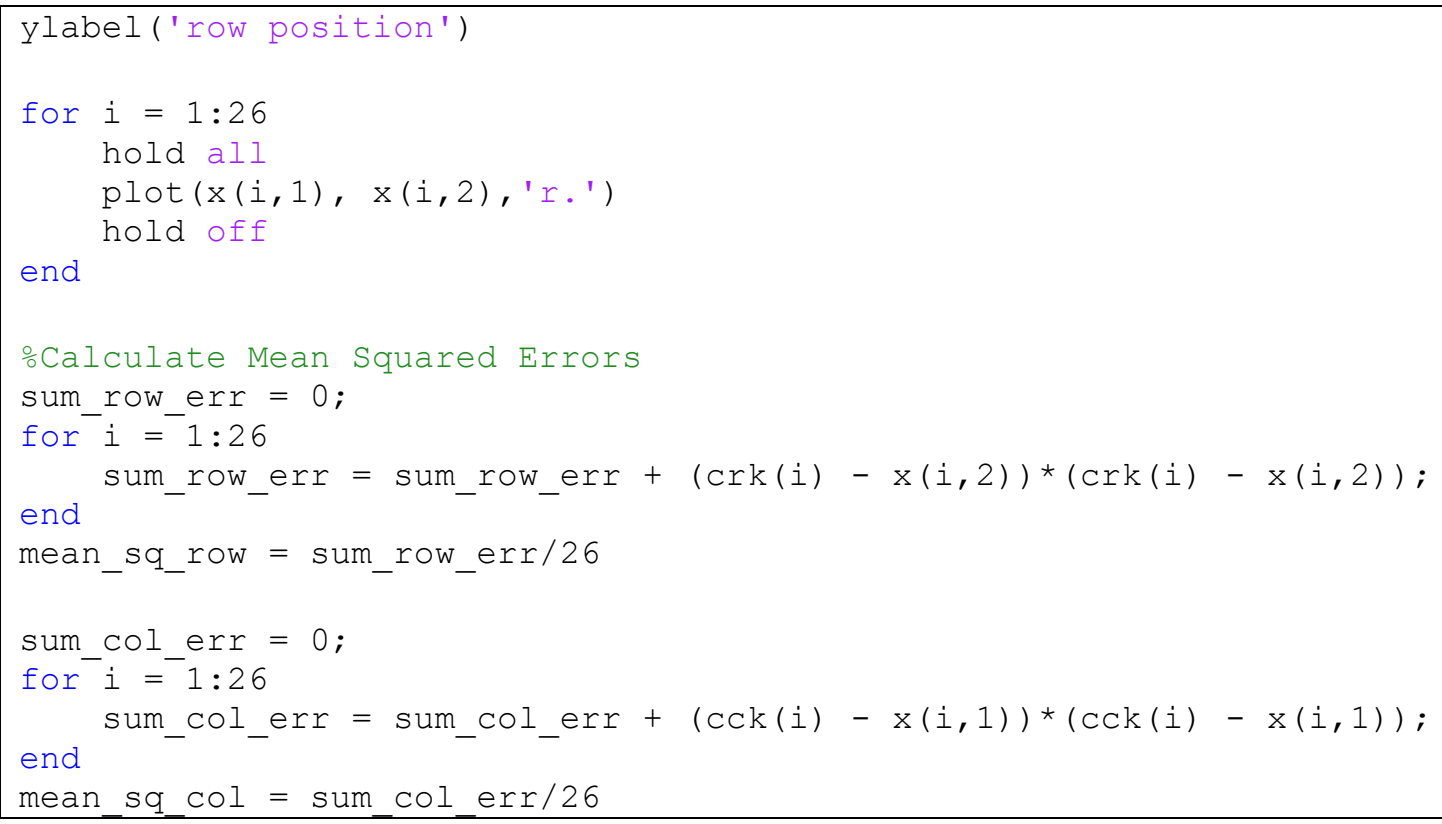

myHinf.m

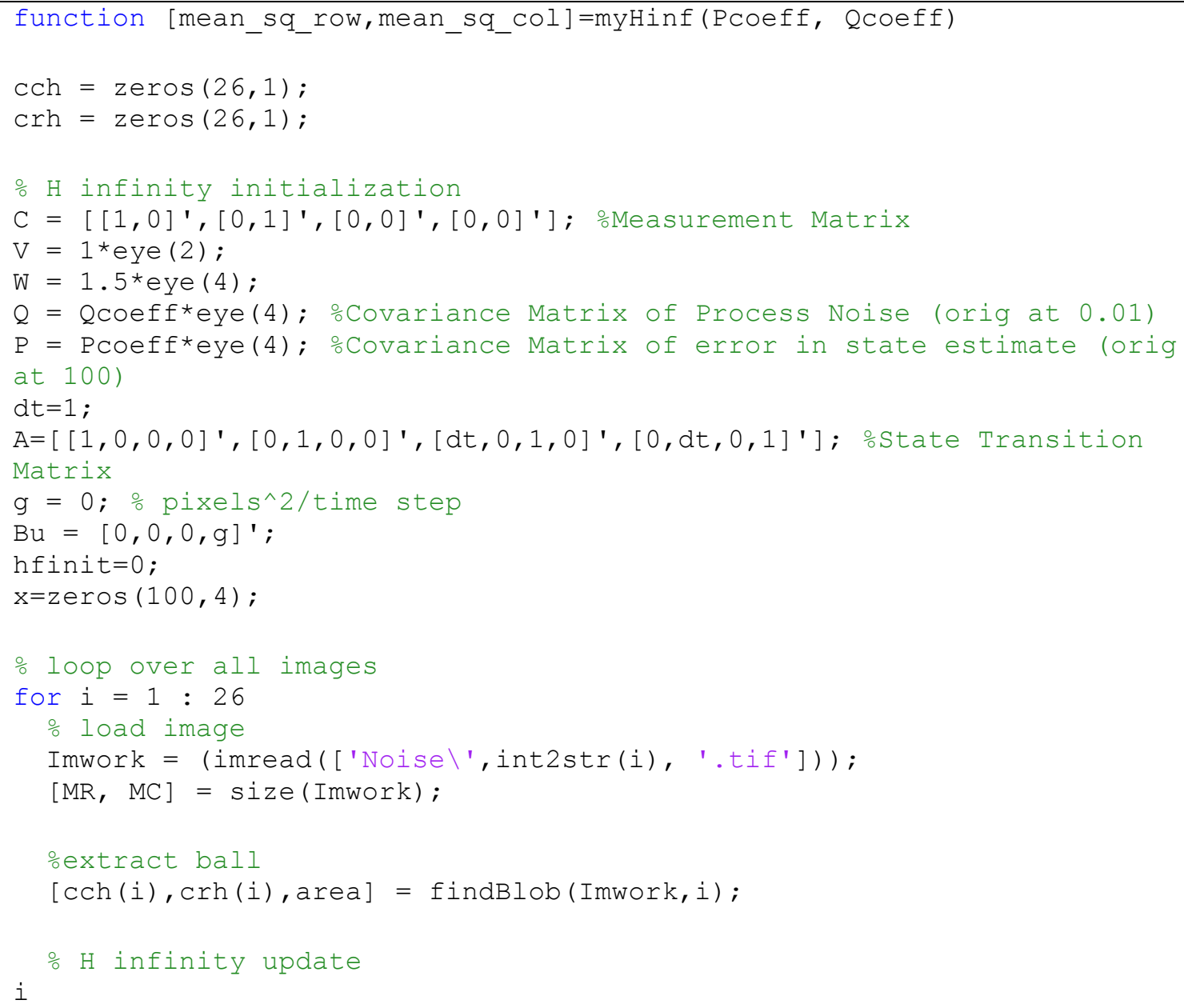




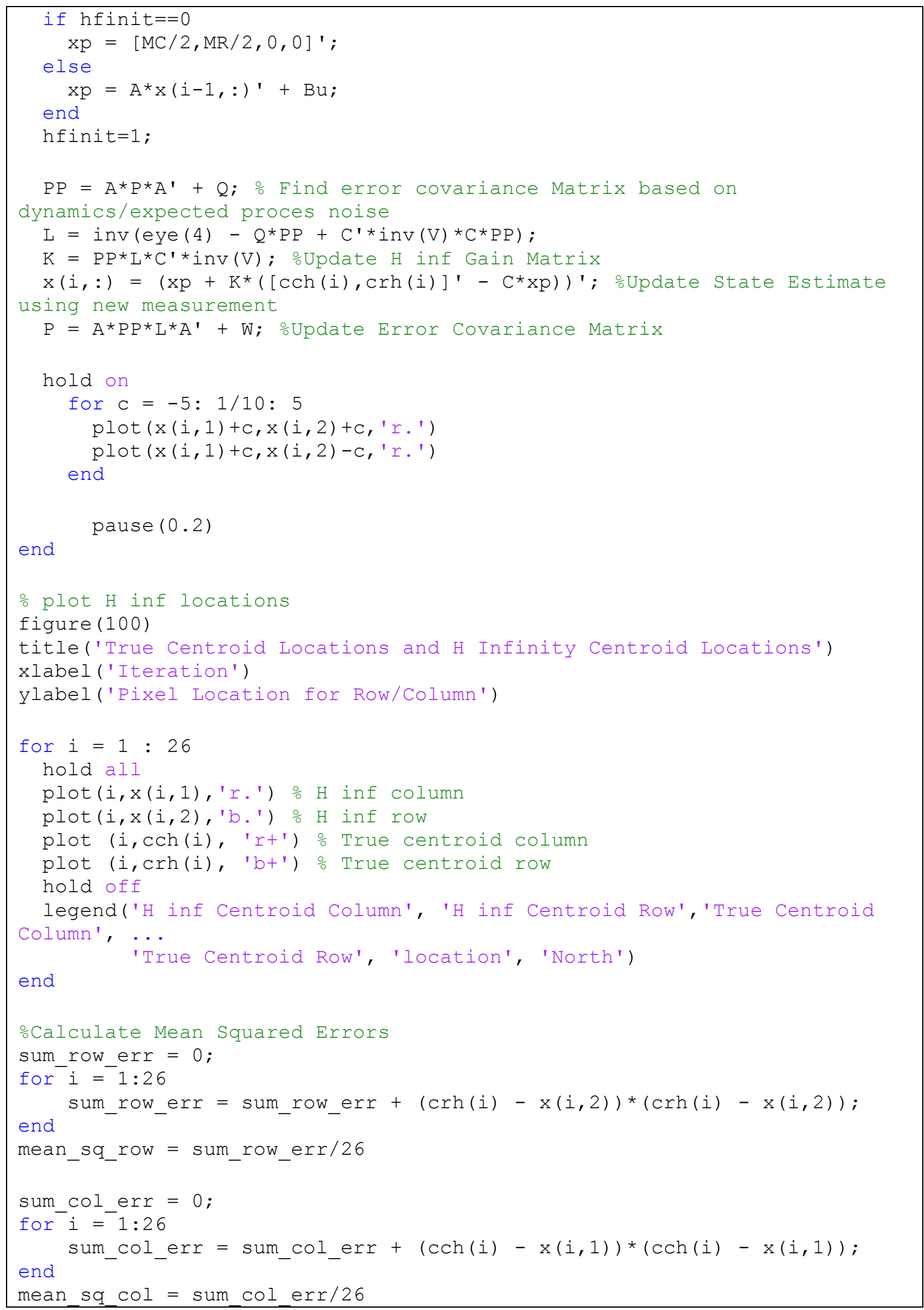


monteCarlo.m

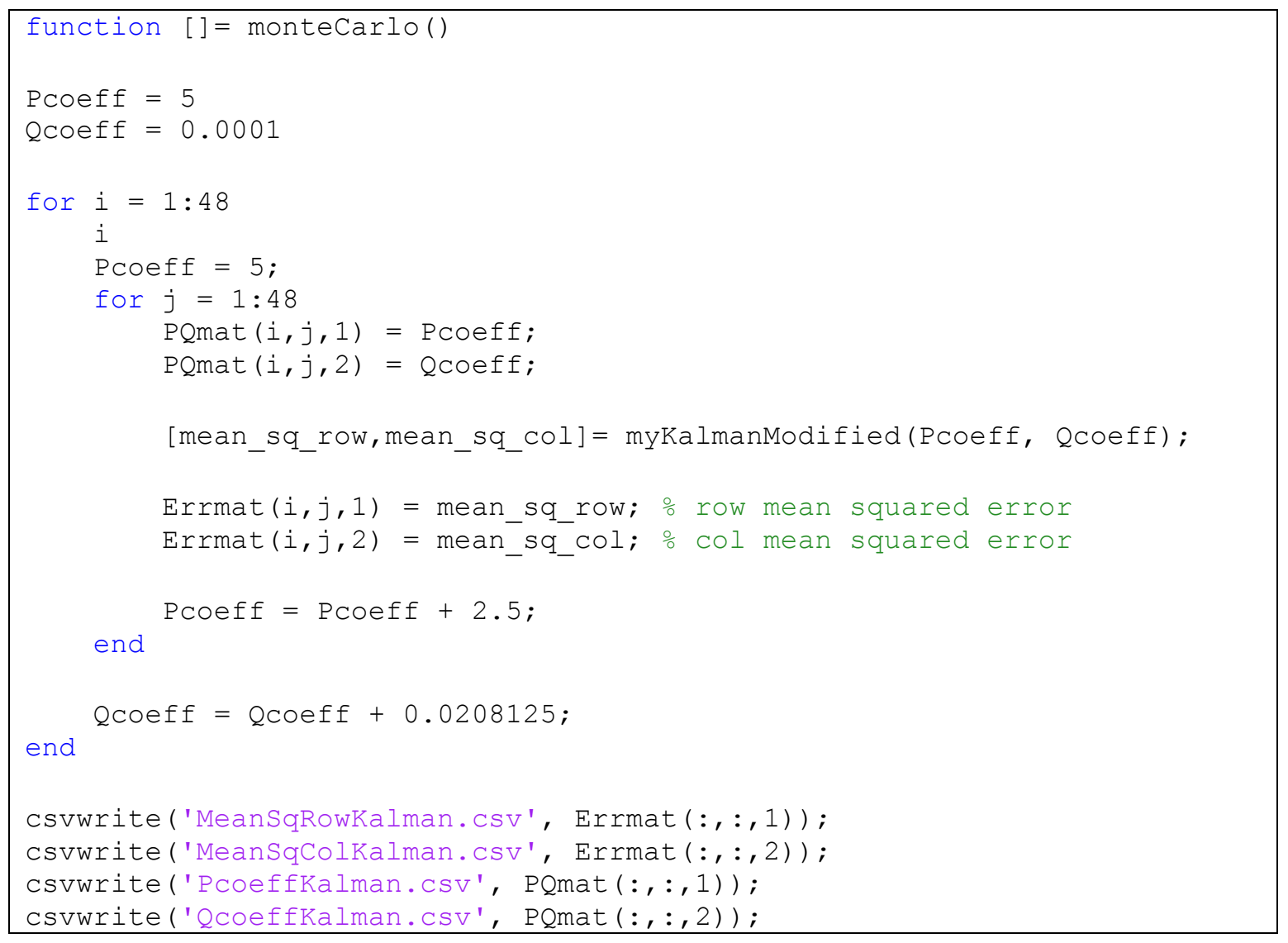

\section{pointTarget.m}

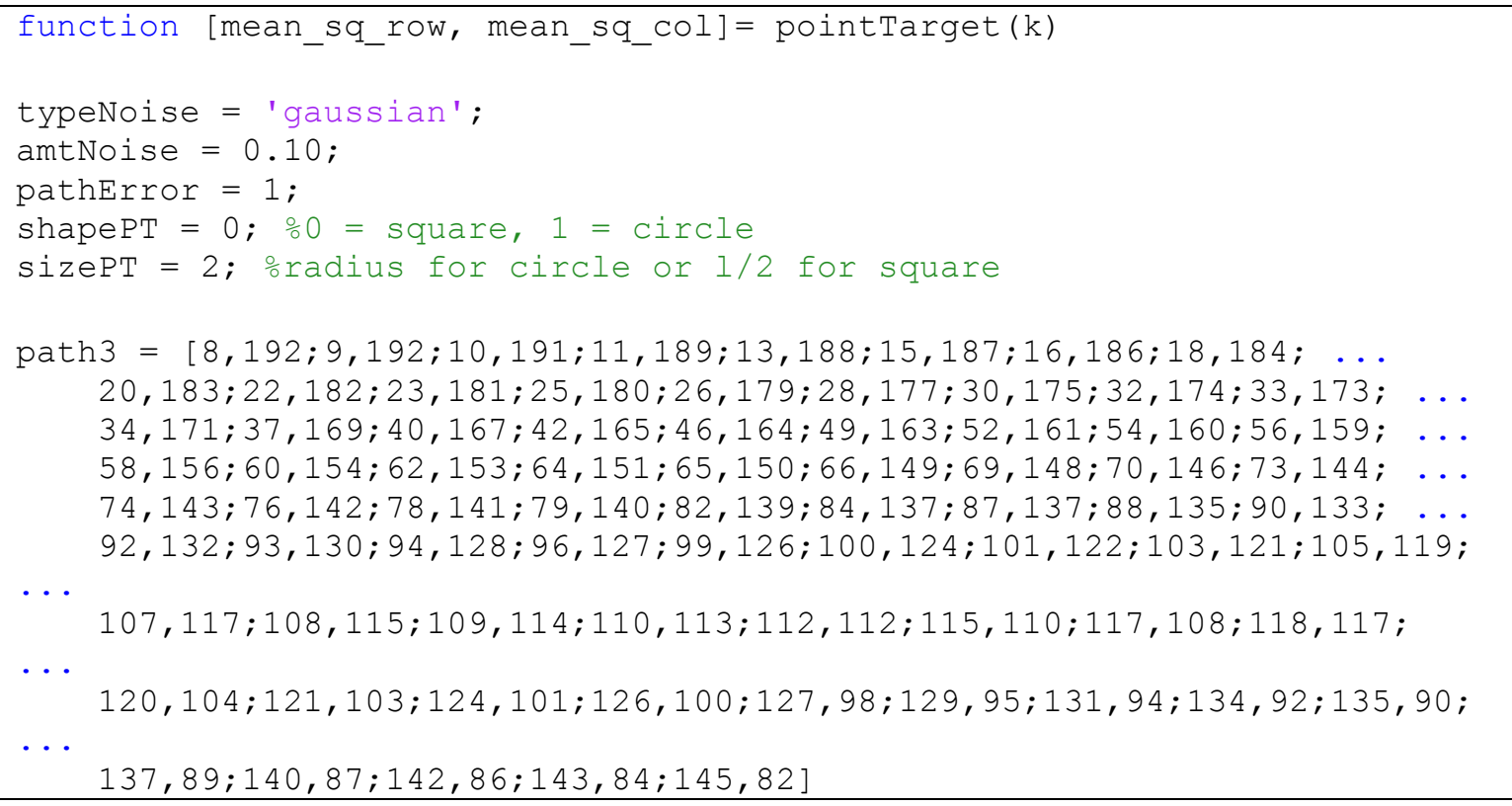




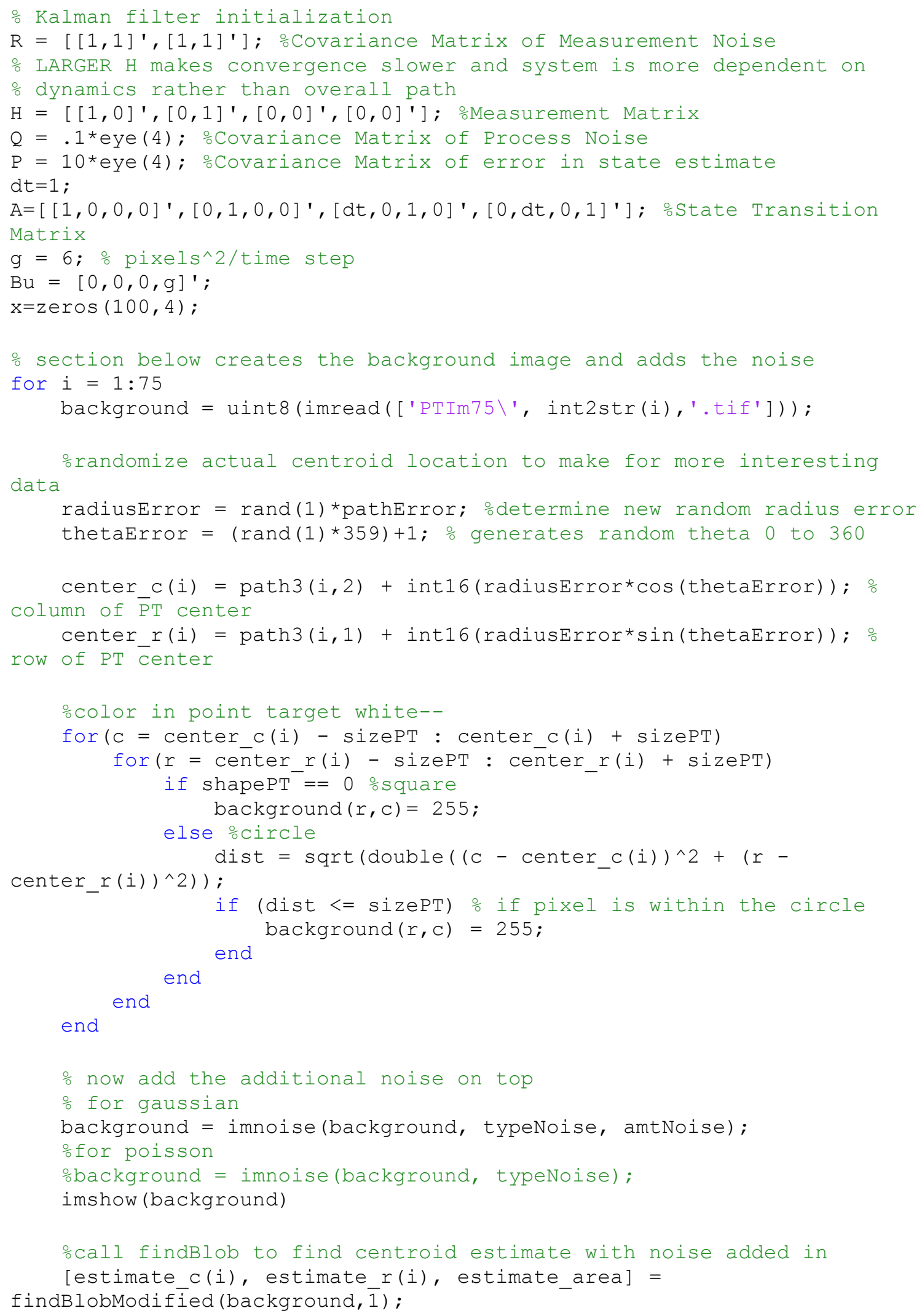




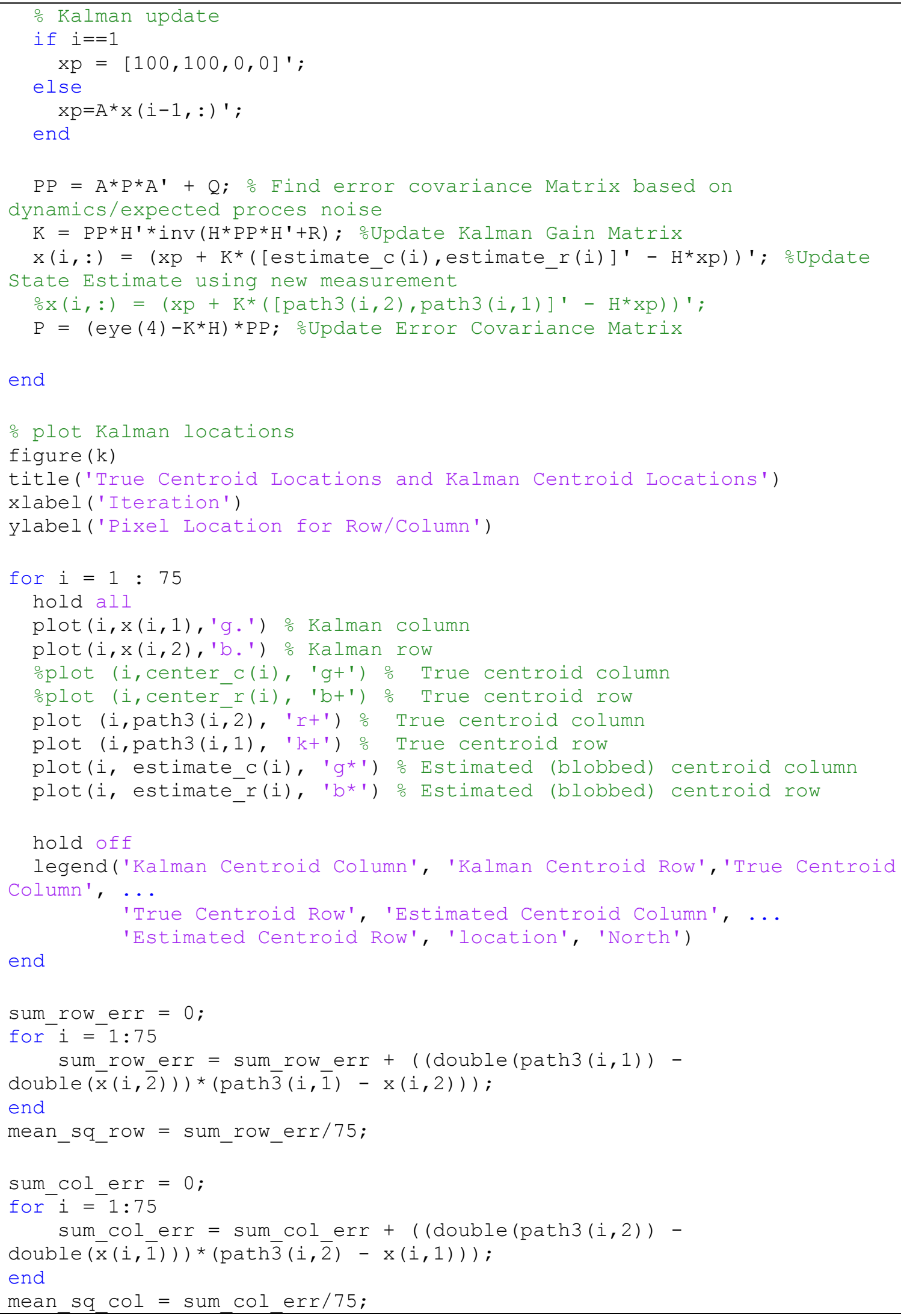


pointTargetHinf.m

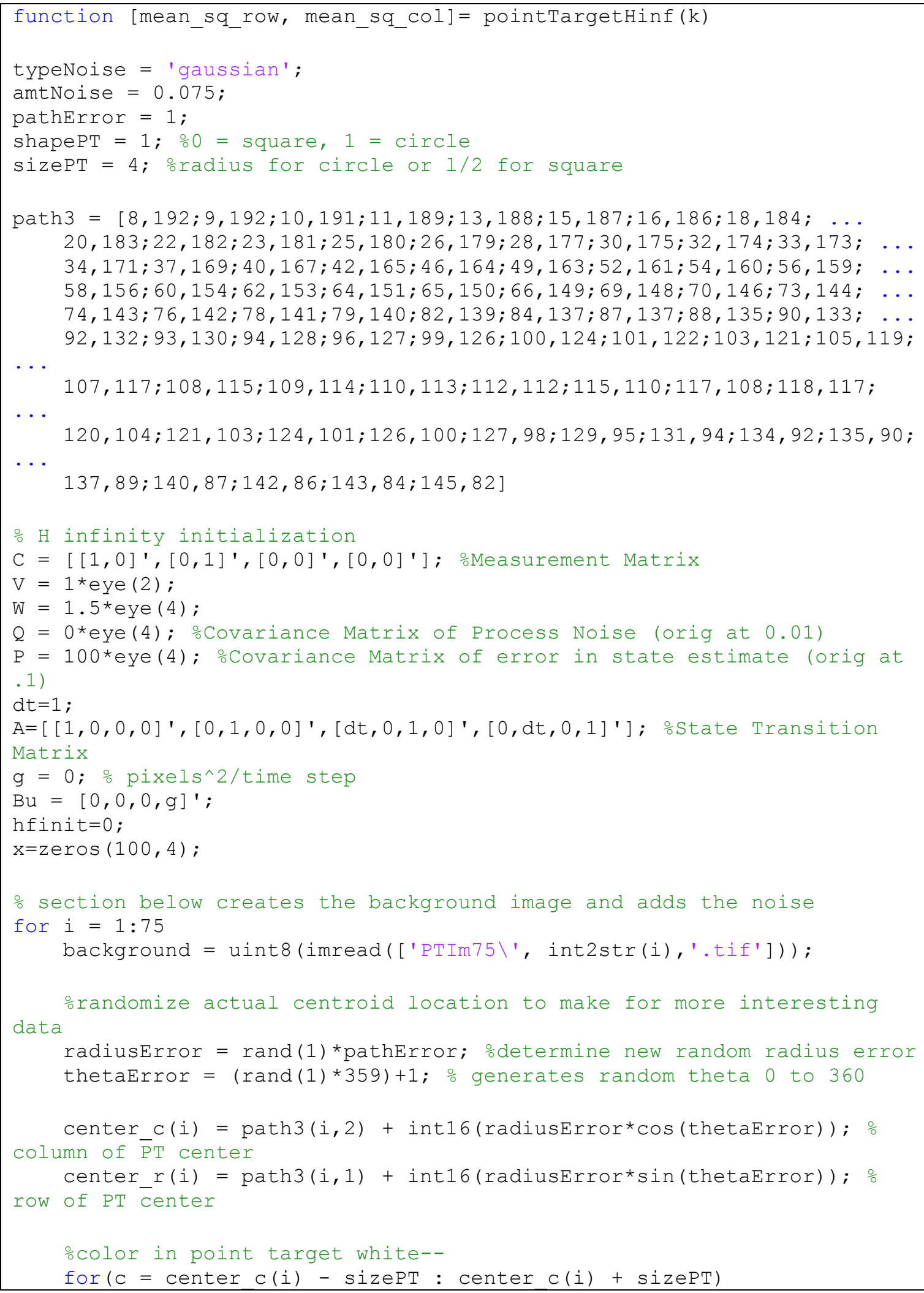




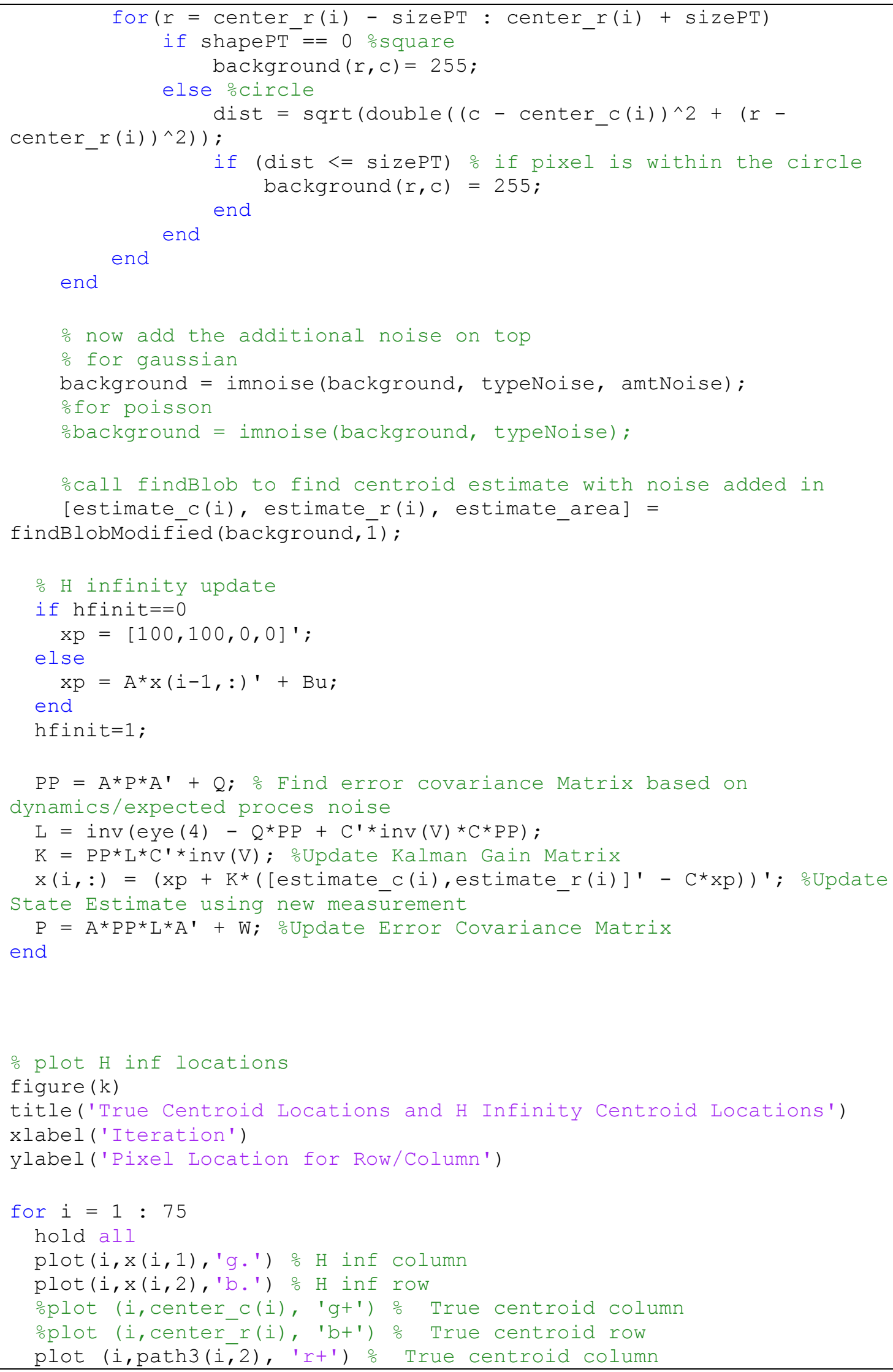




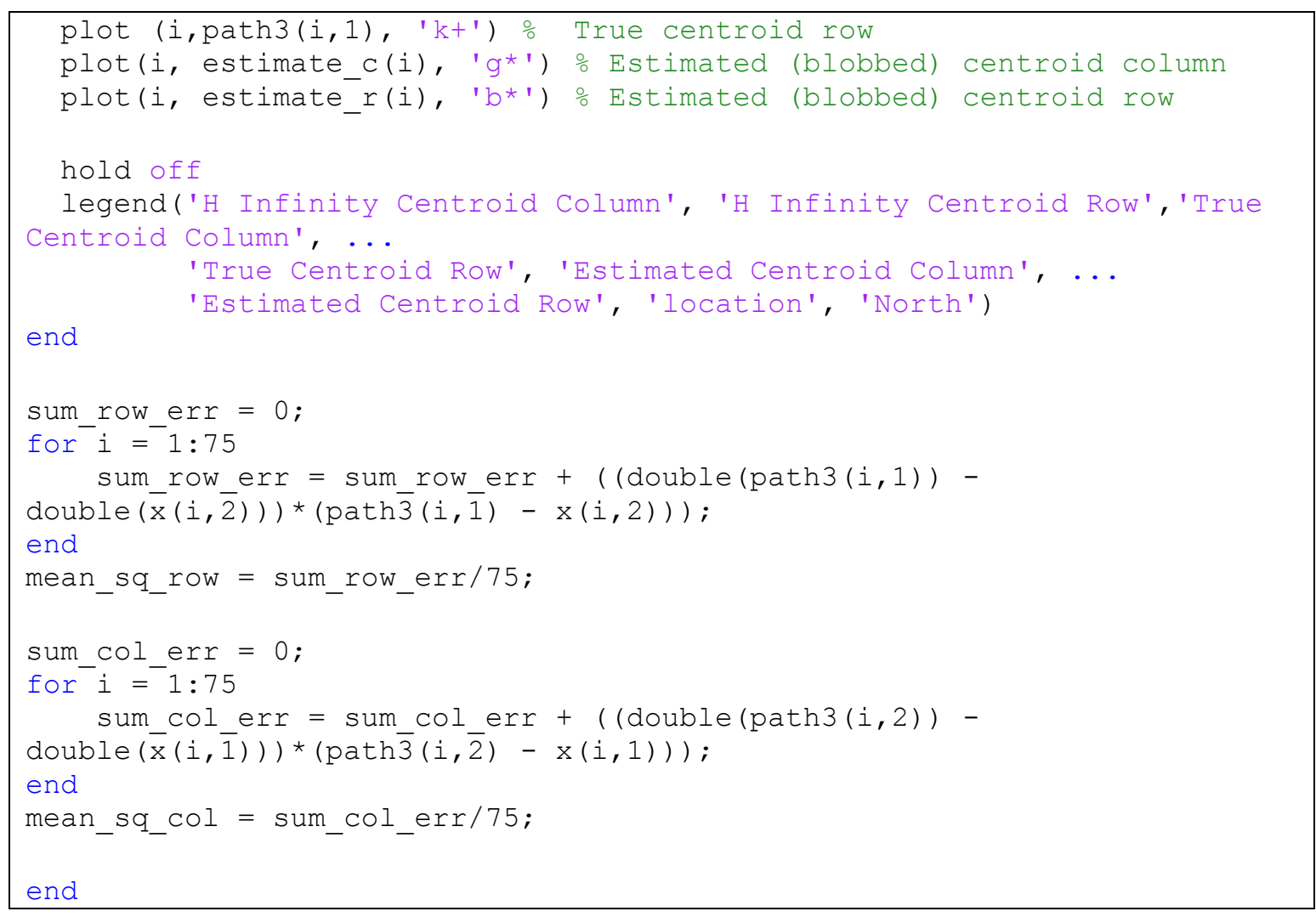

\section{makelmages.m}

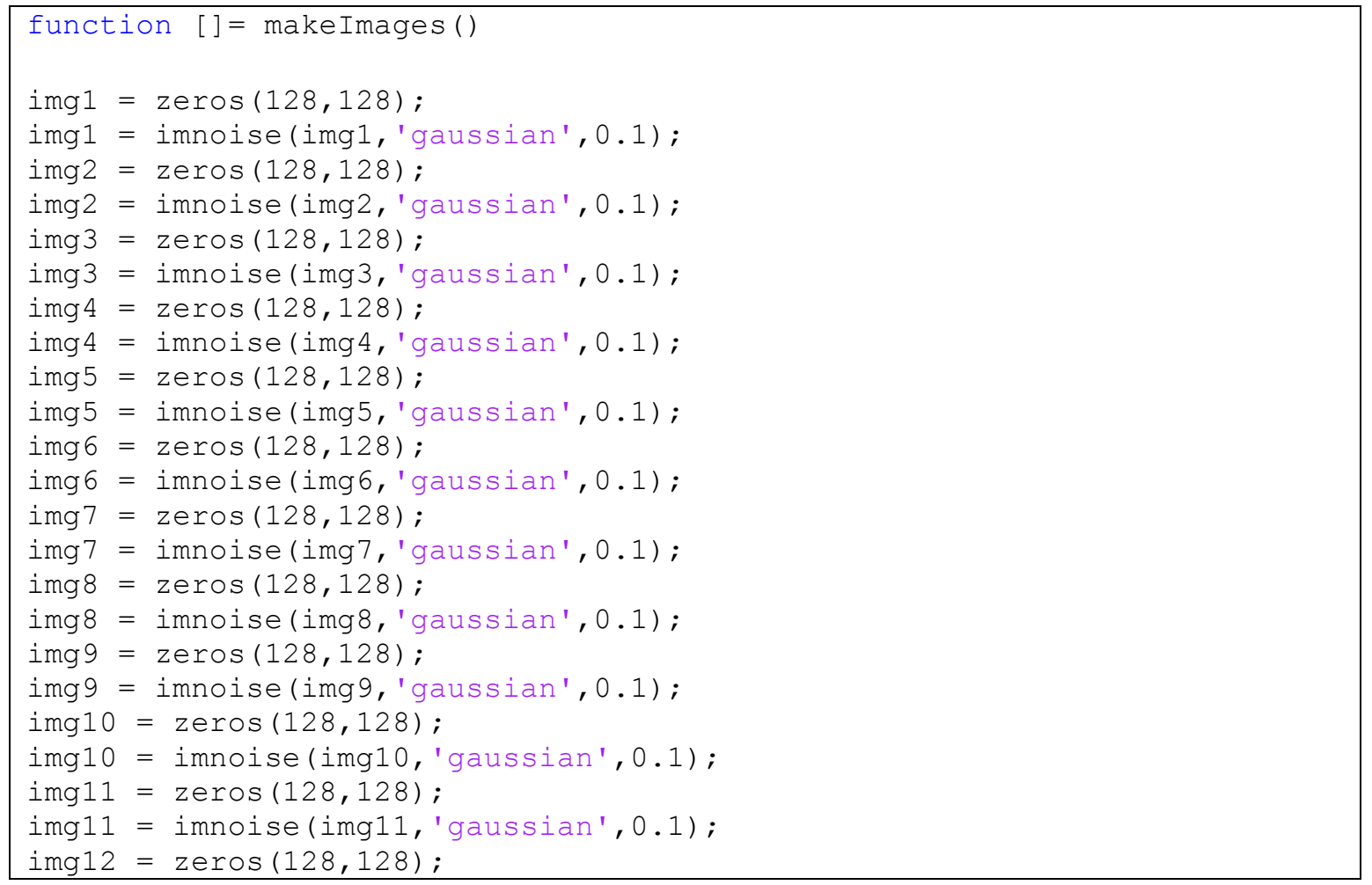




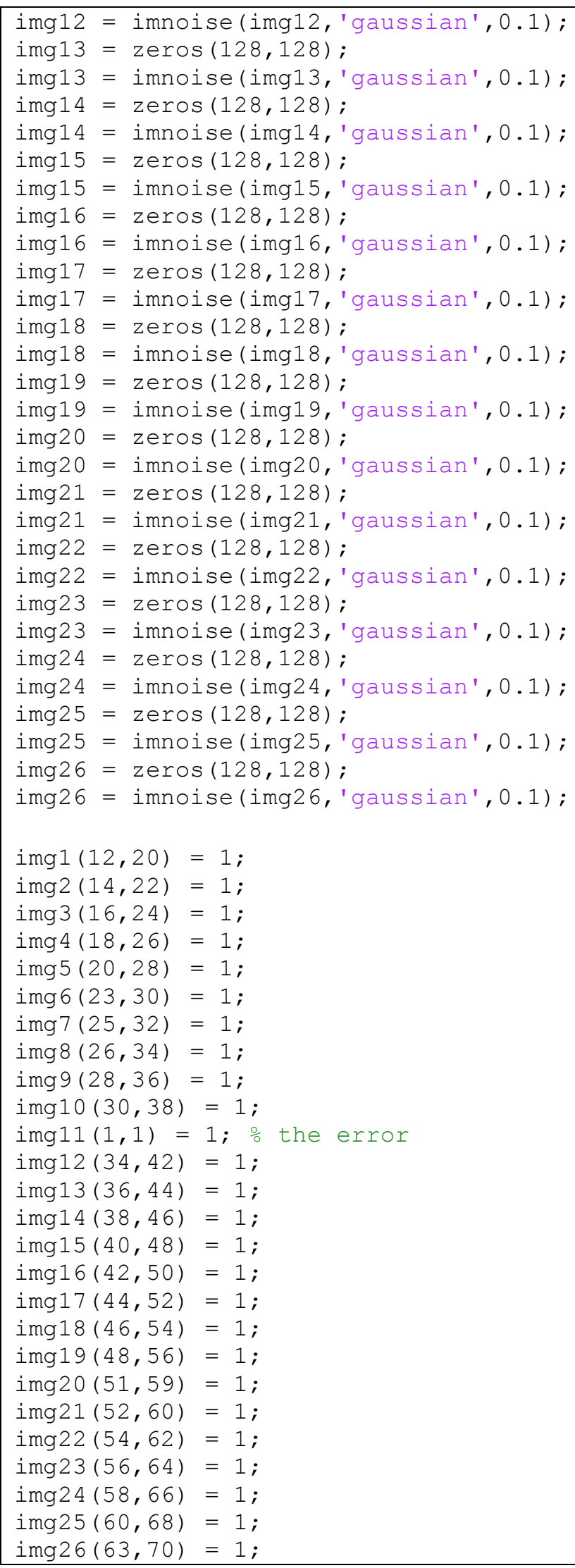




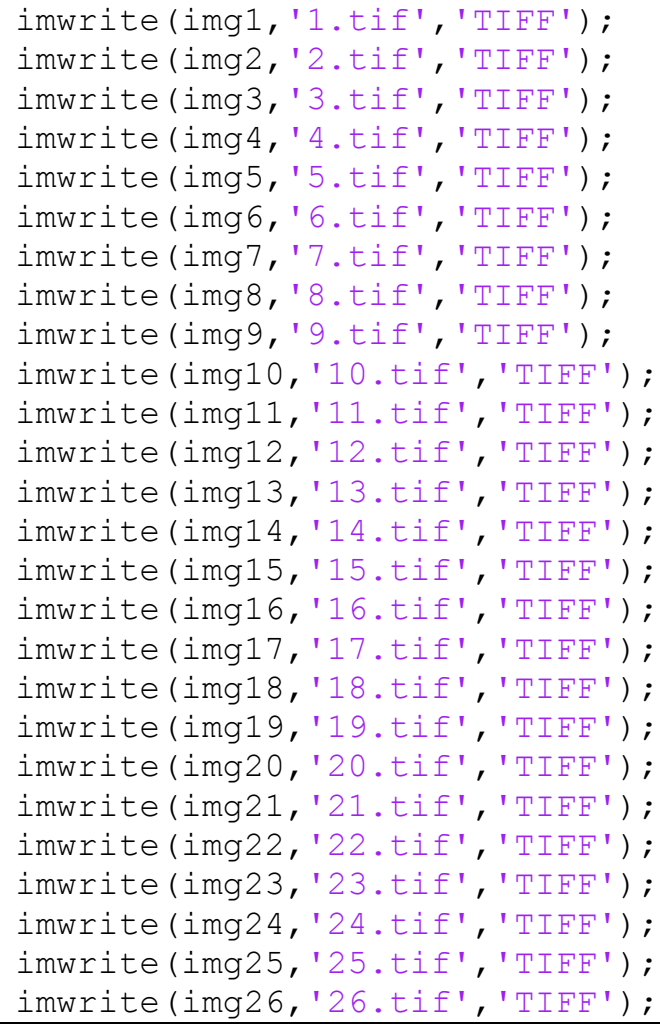

\section{findPixel.m}

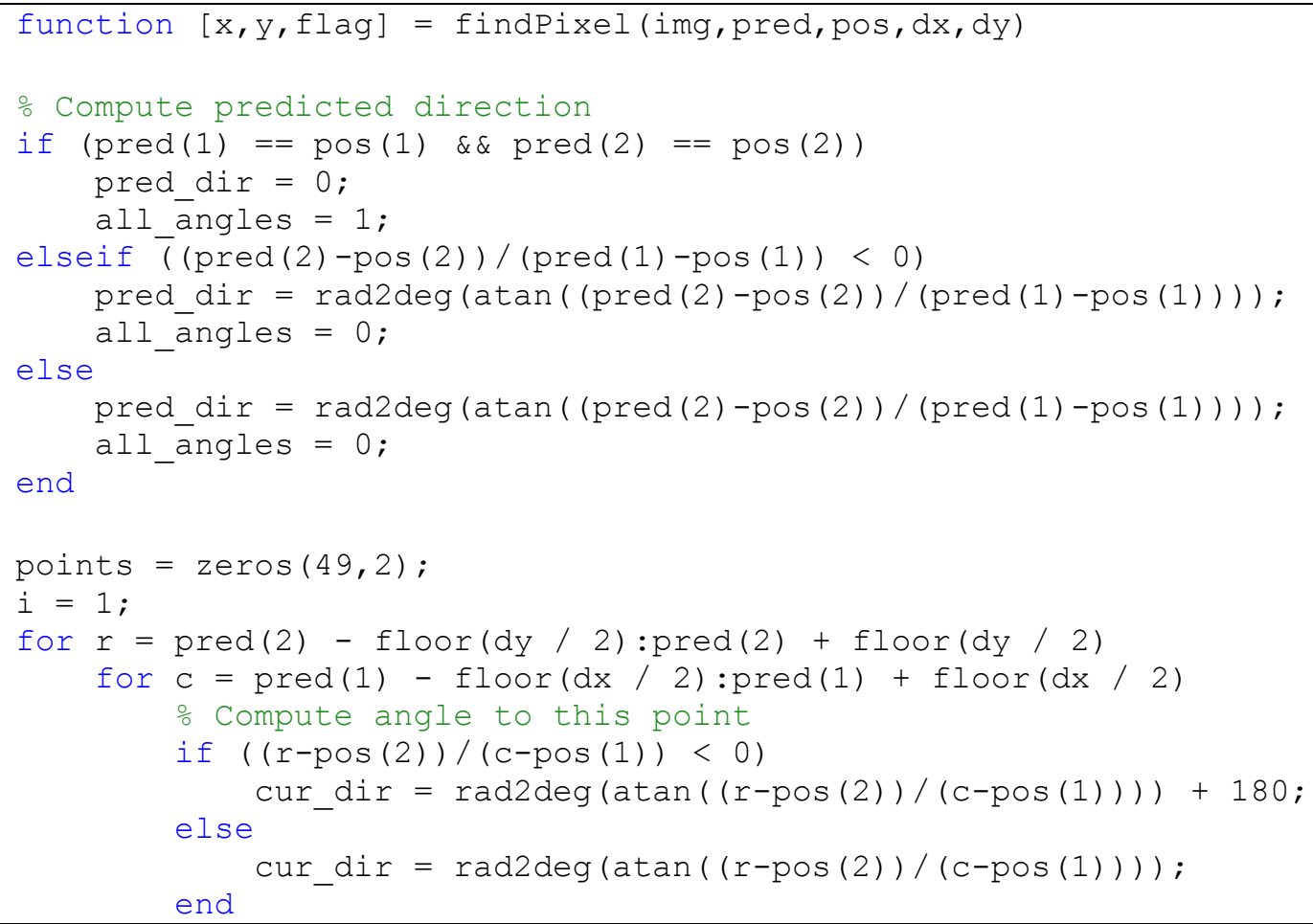




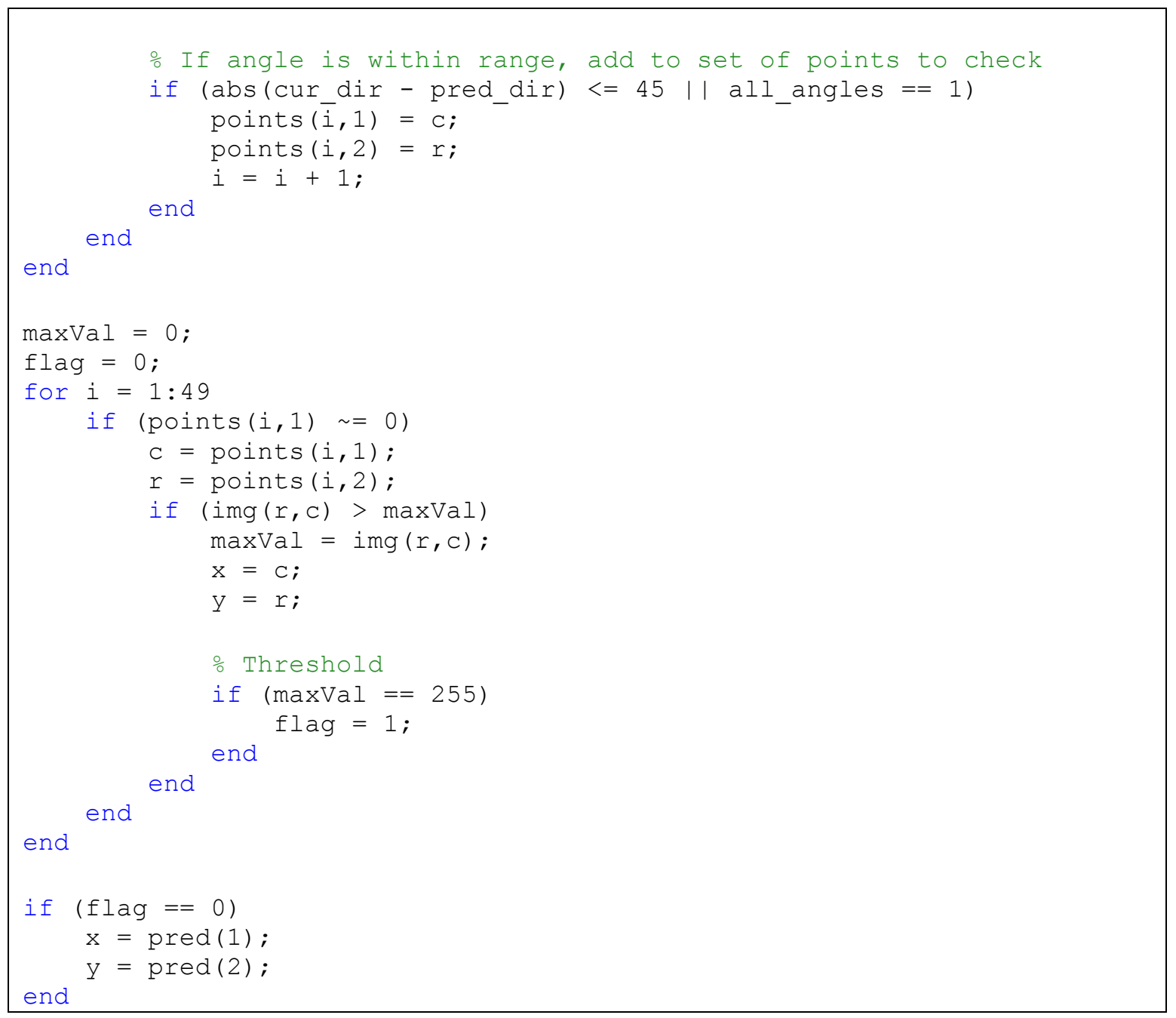

\section{PAMDFilter.m}

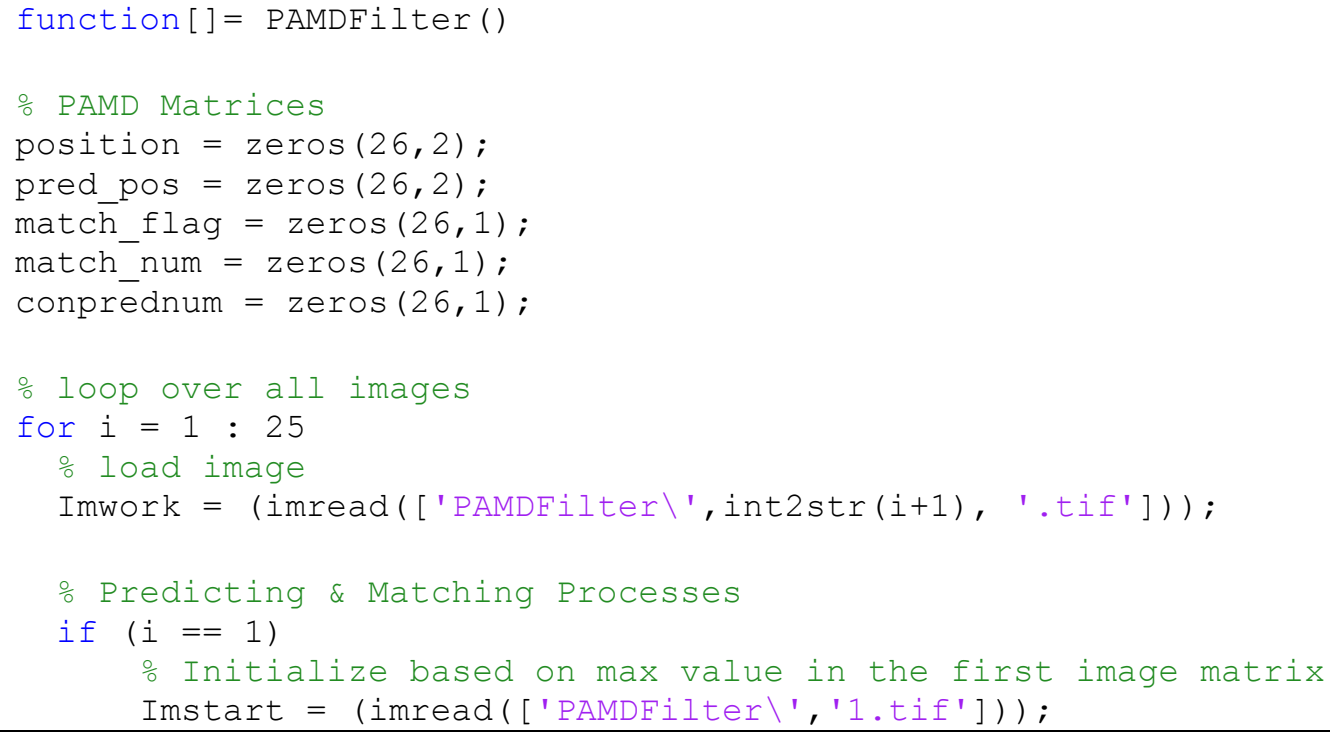




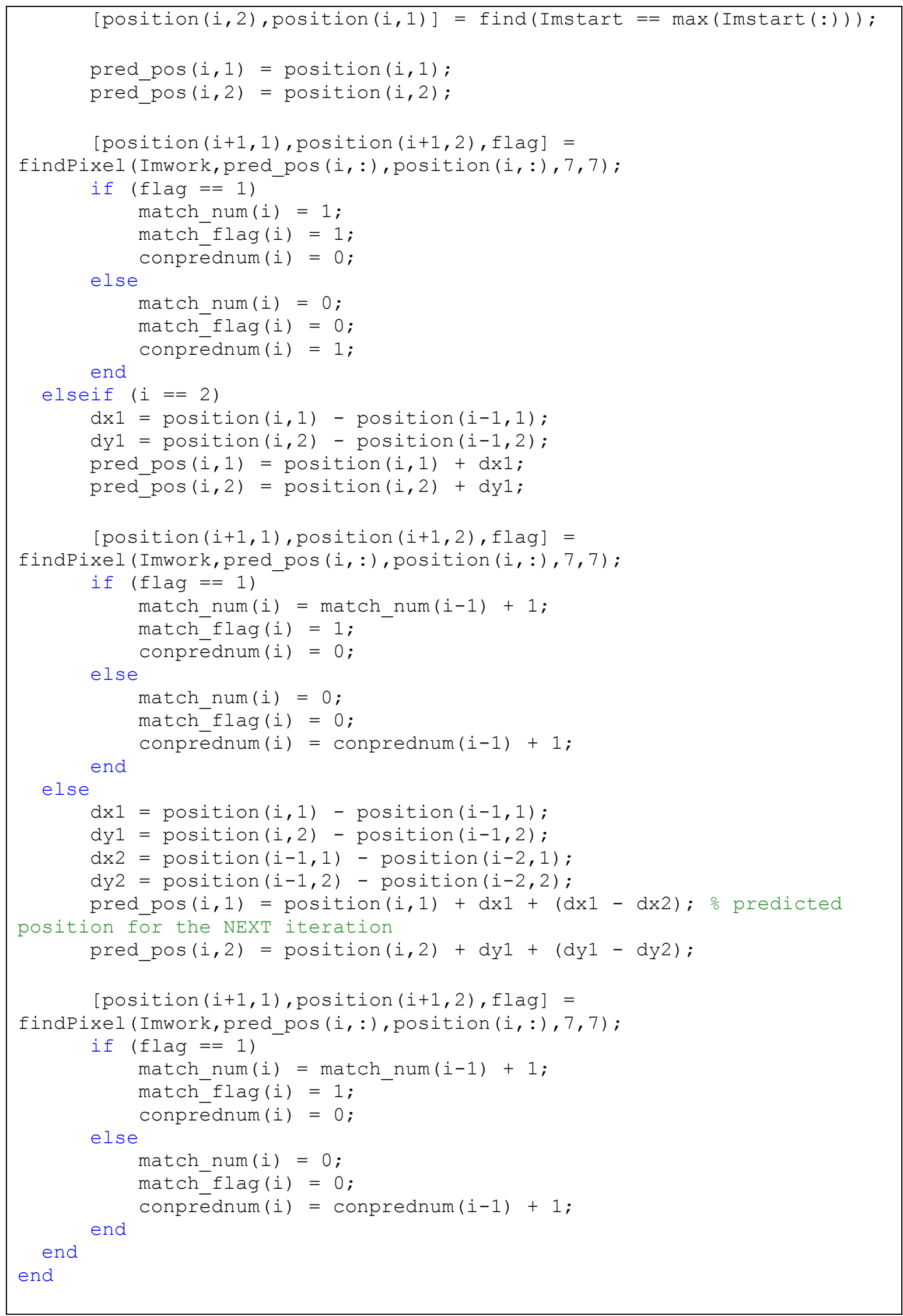


position
plot (position $(:, 1), \operatorname{position}(:, 2)$ ) 